\title{
Anisotropic, Organic Ionic Plastic Crystal Mesophases from Persubstituted Imidazolium Pentacyanocyclopentadienide Salts
}

\author{
Karel Goossens, ${ }^{*},{ }^{\dagger} \nabla$ Lena Rakers, ${ }^{\ddagger, \nabla}$ Benoît Heinrich, ${ }^{\#}$ \\ Guillermo Ahumada, ${ }^{\dagger}{ }^{\dagger} \|$ Takahiro Ichikawa, ${ }^{\perp}$ Bertrand Donnio, ${ }^{\#}$ \\ Tae Joo Shin, ${ }^{\S}$ Christopher W. Bielawski, ${ }^{*}+, \|, \square$ and Frank Glorius ${ }^{*, *}$
}

$†$ Center for Multidimensional Carbon Materials (CMCM), Institute for Basic Science (IBS), Ulsan 44919, Republic of Korea

* Organisch-Chemisches Institut, Westfälische Wilhelms-Universität Münster, Corrensstraße 40, 48149 Münster, Germany

\# Institut de Physique et Chimie des Matériaux de Strasbourg (IPCMS), UMR 7504, CNRS-Université de Strasbourg, 23 rue du Loess, BP43, 67034 Strasbourg Cedex 2, France

\| Department of Chemistry, Ulsan National Institute of Science and Technology (UNIST), Ulsan 44919, Republic of Korea

$\perp$ Department of Biotechnology and Life Science, Tokyo University of Agriculture and Technology, Nakacho, Koganei, Tokyo 184-8588, Japan

$\S$ UNIST Central Research Facilities (UCRF) and School of Natural Science, UNIST, Ulsan 44919, Republic of Korea

Department of Energy Engineering, UNIST, Ulsan 44919, Republic of Korea

* E-mail addresses of the corresponding authors:

karel.cw.goossens@gmail.com,bielawski@unist.ac.kr,glorius@uni-muenster.de

$\nabla$ These authors contributed equally to this work. 


\section{Supporting Information}

\section{Table of Contents}

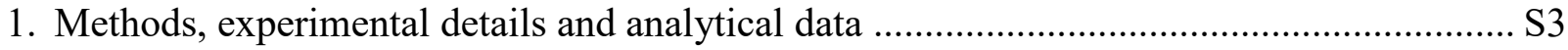

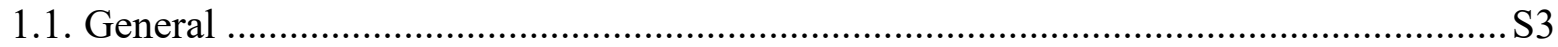

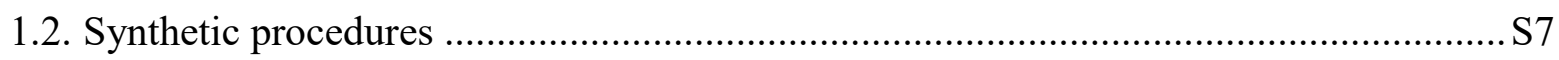

1.2.1. Syntheses of precursors ............................................................................. $\mathrm{S} 7$

1.2.2. Syntheses of iodide salt 5-I-1 and bromide salts $\mathbf{5}-\mathbf{B r}-\boldsymbol{n}(n=7,11,15) \ldots \ldots \ldots . . \mathrm{S} 10$

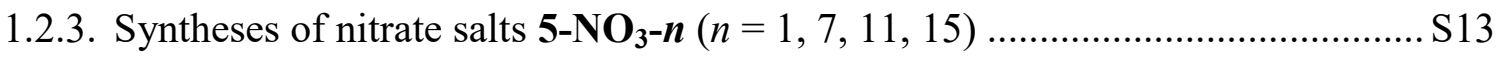

1.2.4. Syntheses of pentacyanocyclopentadienide salts $\mathbf{5 - C p}(\mathbf{C N})_{5}-\boldsymbol{n}(n=1,7$,

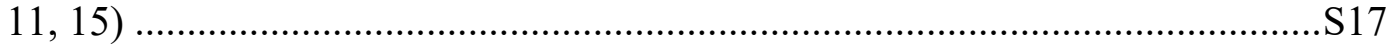

1.2.5. Summary of NMR data ............................................................................ S20

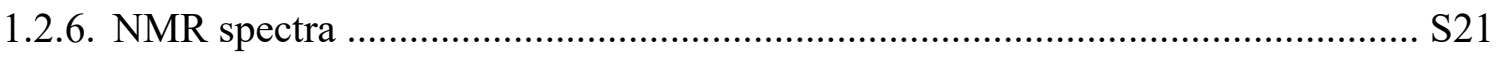

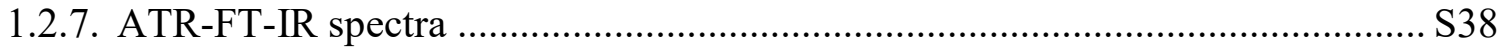

2. Polarized-light optical microscopy (POM) data ……………………………................... S4

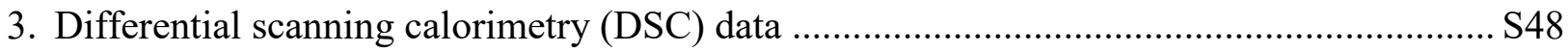

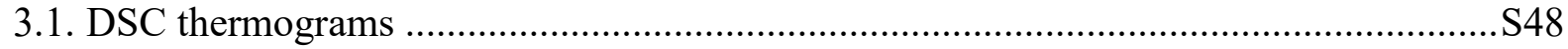

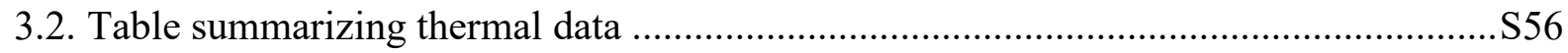

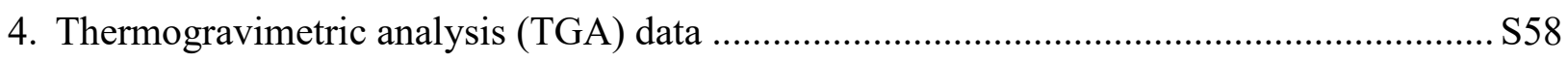

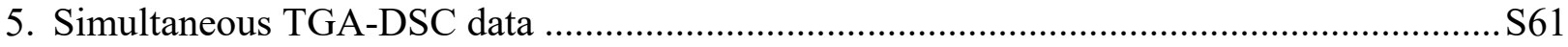

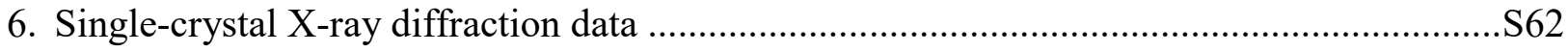

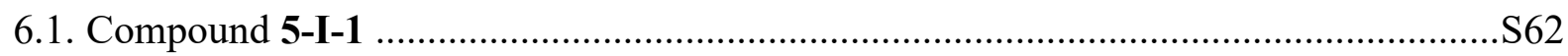

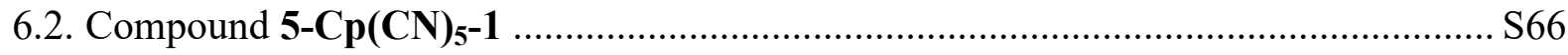

7. Small- to wide-angle X-ray scattering (SWAXS) data ………………………………......... 73

8. Supplementary note: Comparison with the thermal characteristics of long-chain-substi-

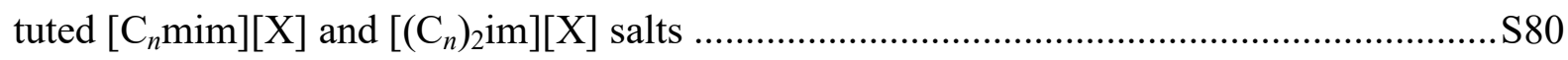

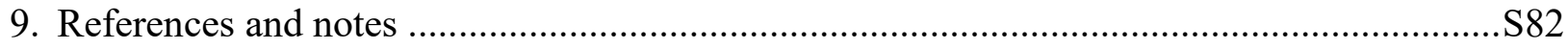




\section{Methods, experimental details and analytical data}

\subsection{General}

The reactions described below were performed in flame-dried or oven-dried glassware under an atmosphere of argon, unless specified otherwise. The given reaction temperatures correspond to the temperatures of the preheated oil baths that surrounded the reaction vessels. Unless specified otherwise, all reagents were purchased from commercial sources (Sigma-Aldrich, Alfa Aesar, Acros Organics, TCI Europe, TCI Japan) and used without further purification. Anhydrous tetrahydrofuran (THF) was obtained by distillation in a continuous still under an atmosphere of argon over $\mathrm{Na}$ /benzophenone as the drying agent. The other anhydrous solvents were purchased from Carl Roth or Sigma-Aldrich, and stored over molecular sieves under an atmosphere of argon. Anhydrous triethylamine was purchased from Sigma-Aldrich, distilled and stored under an atmosphere of argon. The solvents that were used for column chromatography were of technical grade and were flash-distilled prior to use. During the syntheses of the precursors and the halide salts, reaction progress was monitored by thin layer chromatography (TLC) on precoated, aluminum-backed plates (Merck Kieselgel $60 \mathrm{~F}_{254}$ ). TLC spots were visualized by irradiation with a UV lamp and by immersion into a $\mathrm{KMnO}_{4}$ stain. Purification by flash column chromatography was conducted using silica gel (60 A, 35-70 $\mu \mathrm{m}$, Kieselgel, Acros) with a head pressure of argon $(0.1-0.3 \mathrm{~atm})$.

Nuclear magnetic resonance $(\mathrm{NMR})$ spectra were recorded at ambient temperature $(22.0( \pm 1.0)$ ${ }^{\circ} \mathrm{C}$, unless stated otherwise) on a Bruker ARX-300 spectrometer (operating at $300 \mathrm{MHz}$ for ${ }^{1} \mathrm{H}$ ), a Bruker AV-300 spectrometer (operating at $300 \mathrm{MHz}$ for ${ }^{1} \mathrm{H}$ ), a Bruker AV-400 spectrometer (operating at $400 \mathrm{MHz}$ for ${ }^{1} \mathrm{H}$ ) or a Bruker Avance III HD spectrometer (operating at $400 \mathrm{MHz}$ for $\left.{ }^{1} \mathrm{H}\right)$. Chemical shifts $\left(\delta_{\mathrm{H}}\right.$ for ${ }^{1} \mathrm{H}$ and $\delta_{\mathrm{C}}$ for ${ }^{13} \mathrm{C}\left\{{ }^{1} \mathrm{H}\right\}$ ) are given in parts per million (ppm) relative to tetramethylsilane (TMS) using the residual, non-deuterated solvent as an internal standard. For ${ }^{1} \mathrm{H}$ NMR: $\mathrm{CDCl}_{3}, 7.26$ ppm; $\mathrm{CD}_{2} \mathrm{Cl}_{2}, 5.32 \mathrm{ppm}$; DMSO- $d_{6}, 2.50$ ppm. ${ }^{1}$ For ${ }^{13} \mathrm{C}$ NMR: $\mathrm{CDCl}_{3}, 77.16$ ppm; $\mathrm{CD}_{2} \mathrm{Cl}_{2}, 53.84$ ppm; DMSO- $d_{6}, 39.52$ ppm. ${ }^{1}$ Abbreviations used for the description of the spectra are: $\mathrm{s}=$ singlet, $\mathrm{d}=$ doublet, $\mathrm{t}=$ triplet, $\mathrm{q}=$ quartet, quint $=$ quintet, $\mathrm{dd}=$ doublet of doublets, and $\mathrm{m}=$ multiplet. Coupling constants $(J)$ are expressed in hertz $(\mathrm{Hz})$. 
Fourier-transform infrared (FT-IR) spectra were recorded in attenuated total reflectance (ATR) mode on an Agilent Cary 630 FT-IR spectrometer using a germanium crystal (Ge-ATR). Abbreviations used for the description of the spectra are: $\mathrm{vs}=$ very strong, $\mathrm{s}=$ strong, $\mathrm{m}=$ medium, and $\mathrm{w}=$ weak; $v_{\mathrm{s}}=$ symmetric stretching vibration, and $v_{\mathrm{as}}=$ asymmetric stretching vibration.

High resolution mass spectra (HRMS) were recorded in electrospray ionization (ESI) mode on a Bruker Daltonics MicroTof mass spectrometer, a Thermo Scientific LTQ Orbitrap XL mass spectrometer or a Waters Xevo G2-XS QTof mass spectrometer. The Bruker and Thermo Scientific instruments were calibrated using sodium formate clusters; the Waters instrument was calibrated using an acetonitrile solution of leucine-enkephalin $+1 \%$ formic acid and using an aqueous solution of sodium formate $+1 \%$ formic acid.

Elemental analyses (carbon, hydrogen, nitrogen, and sulfur) were performed using a Thermo Scientific Flash 2000 Organic Elemental Analyzer.

Optical textures were observed using an Olympus BX53-P polarized-light optical microscope that was equipped with a rotatable graduated sample platform and an Instec HCS402 dual heater temperature stage. The latter was equipped with a precision $\mathrm{XY}$ positioner and coupled to an Instec $\mathrm{LN}_{2}$-SYS liquid nitrogen cooling system and an Instec mK2000 programmable temperature controller. Images were recorded with a QImaging Retiga 2000R CCD camera that was coupled to the microscope via a $\mathrm{C}$ mount. The samples were pressed between an untreated microscopy glass slide and glass coverslip $(0.13-0.17 \mathrm{~mm}$ thick, Duran) prior to analysis.

DSC data were recorded under nitrogen $\left(50 \mathrm{~mL} \mathrm{~min}^{-1}\right)$ on a TA Instruments DSC Q2000 module equipped with an RCS90 cooling system at a heating rate of $10{ }^{\circ} \mathrm{C} \mathrm{min}{ }^{-1}$ and a cooling rate of 5 ${ }^{\circ} \mathrm{C} \min ^{-1}$. The quantity of sample analyzed was typically $4-5 \mathrm{mg}$. A small hole was pierced into the lid of the aluminum sample pans. The measurements were performed using the Tzero ${ }^{\mathrm{TM}} \mathrm{Heat}$ Flow T4P option. High-purity sapphire disks were used for the Tzero ${ }^{\mathrm{TM}}$ calibration and highpurity indium was used as a standard for temperature and enthalpy calibrations $\left(T_{\mathrm{m}}=156.60{ }^{\circ} \mathrm{C},{ }^{2}\right.$ $\Delta H_{\text {fus }}=28.66 \mathrm{~J} \mathrm{~g}^{-1}{ }^{3}$ ). DSC data analysis was performed with the Universal Analysis 2000 
software (version 4.5A) from TA Instruments. The abbreviations used to describe the thermal phase properties are explained in the main text and in the captions of the figures and tables.

TGA data were recorded under nitrogen $\left(60 \mathrm{~mL} \mathrm{~min}^{-1}\right)$ on a TA Instruments TGA Q500 module at a heating rate of $5{ }^{\circ} \mathrm{C} \mathrm{min}^{-1}$ and using a platinum sample pan. The quantity of sample analyzed was typically 5-8 mg. High-purity nickel was used as a standard for temperature calibration (based on its Curie temperature of $358.28^{\circ} \mathrm{C}^{4}$ ).

Simultaneous TGA-DSC measurements were performed using a TA Instruments SDT Q600 module at a heating rate of $10{ }^{\circ} \mathrm{C} \mathrm{min}^{-1}$ and under nitrogen $\left(100 \mathrm{~mL} \mathrm{~min}{ }^{-1}\right)$. An alumina sample pan was used. The quantity of sample analyzed was approximately $6.5 \mathrm{mg}$. High-purity zinc was used as a standard for temperature calibration, and sapphire was used as a standard for heat flow calibration.

To determine the crystal structures of compounds 5-I-1 and 5-Cp(CN) $\mathbf{5}-\mathbf{1}$, X-ray intensity data were collected at $123 \mathrm{~K}$ on a Rigaku XtaLAB P200 diffractometer equipped with a Pilatus 200K detector, using $\omega$ scans and $\mathrm{Cu} \mathrm{K \alpha}$ radiation (wavelength $\lambda=1.54187 \AA$ ). See Section 6 for further information.

Most of the SWAXS patterns were obtained with a transmission Guinier-like geometry. A linear focalized monochromatic $\mathrm{Cu} \mathrm{K \alpha l}$ beam $(\lambda=1.5405 \AA)$ was obtained using a sealed-tube generator $(600 \mathrm{~W})$ equipped with a bent quartz monochromator. In all cases, the crude powder was filled in Lindemann capillaries of $1 \mathrm{~mm}$ diameter and $10 \mu \mathrm{m}$ wall thickness or in homemade sealed cells of $1 \mathrm{~mm}$ pathlength and equipped with $11 \mu \mathrm{m}$-thick aluminum windows. The diffraction patterns were recorded with a curved Inel CPS120 counter gas-filled detector linked to a data acquisition computer (periodicities up to $90 \AA$ ) and on image plates scanned by Amersham Typhoon IP with $25 \mu \mathrm{m}$ resolution (periodicities up to $120 \AA$ ). The sample temperature could be varied between $20^{\circ} \mathrm{C}$ and $200{ }^{\circ} \mathrm{C}$ with a precision of $\pm 0.01{ }^{\circ} \mathrm{C}$, and exposure times varied from 1 to 5 hours.

Synchrotron-based X-ray scattering measurements of the mesophase adopted by compound 5$\mathbf{C p}(\mathbf{C N})_{5}-1$ were performed at the PLS-II 6D UNIST-PAL Beamline of the Pohang Accelerator Laboratory (PAL), Pohang, Republic of Korea. The X-rays coming from the bending magnet 
were monochromated using $\operatorname{Si}(111)$ double crystals and focused at the detector position by the combination of a second, sagittal-type monochromator crystal and a toroidal mirror system. The diffraction patterns were recorded by a Rayonix MX225-HS 2D CCD detector $\left(225 \times 225 \mathrm{~mm}^{2}\right.$ square active area, full resolution $5760 \times 5760$ pixels) with $2 \times 2$ binning. The peak positions in the $1 \mathrm{D}$ intensity profiles, which were obtained from azimuthal averaging of the $2 \mathrm{D}$ patterns, were used for phase type assignments. SWAXS patterns were recorded using $12.3984 \mathrm{keV}$ X-ray radiation (wavelength $\lambda=1.00 \AA$ ) and a sample-to-detector distance (SDD) of ca. $436 \mathrm{~mm}$. Diffraction angles were calibrated using a lanthanum hexaboride $\left(\mathrm{LaB}_{6}\right)$ standard (NIST SRM 660c). Samples were contained in a borosilicate glass (glass \#50) capillary with an outer diameter of $0.8 \mathrm{~mm}$ and a wall thickness of $10 \mu \mathrm{m}$ and were irradiated for 5-10 seconds per measurement, depending on the saturation level of the detector. The capillaries were inserted into a custommade brass holder that was placed into a Linkam TS1500V temperature stage to achieve temperature control. The samples were allowed to equilibrate at each temperature before starting a measurement.

Dynamic ionic conductivity data were recorded with a Solartron (Schlumberger) SI 1260 impedance/gain-phase analyzer at a cooling rate of $2{ }^{\circ} \mathrm{C} \min ^{-1}$, using a frequency range of $100 \mathrm{~Hz}$ $-20 \mathrm{MHz}$ and a voltage of $0.6 \mathrm{~V}$. The temperature was controlled using a Mettler-Toledo HS82 dual heater temperature stage that was coupled to a Mettler-Toledo HS1 programmable temperature controller. A glass sample cell with comb-shaped gold electrodes with thicknesses of $0.8 \mu \mathrm{m}$ and spacings of $300 \mu \mathrm{m}$ was used for all the measurements. The cell constant was determined using an aqueous $\mathrm{KCl}$ solution $(0.01 \mathrm{M})$. Mixtures of $\mathbf{5}-\mathbf{C p}(\mathbf{C N})_{5} \mathbf{- 1}$ and $\mathrm{Na}\left[\mathrm{Cp}(\mathrm{CN})_{5}\right]$ were prepared by slowly evaporating acetonitrile solutions of the two components followed by heating the samples to their isotropic liquid states.

Molecular models were created using the Chem3D Pro 15.1 software package (PerkinElmer Informatics, Inc.). 


\subsection{Synthetic procedures}

\subsubsection{Syntheses of precursors}

Precursors 1-octanal (1-7) and 1-dodecanal (1-11) were purchased from commercial sources (Acros Organics, Sigma-Aldrich). Precursor 1-hexadecanal (1-15) is commercially available but was prepared as described elsewhere. ${ }^{5}$ Precursors $\mathbf{2}-\boldsymbol{n}(n=7,11,15)$ and $\mathbf{3}-\boldsymbol{n}(n=7,11,15)$ were also prepared as described elsewhere. ${ }^{5}$

General method for the synthesis of imidazole precursors $4-n(n=7,11,15)$

A 1,2-diketone 3-n (1 equiv.), aldehyde 1-n (1.2 equiv.) and $\mathrm{NH}_{4} \mathrm{OAc}$ (2.4 equiv.) were dissolved in dry ethanol $([1,2$-diketone $]=0.5 \mathrm{M})$ in a flame-dried Schlenk pressure tube, after which 3 drops of HOAc were added. The reaction mixture was stirred for $18 \mathrm{~h}$ at $110{ }^{\circ} \mathrm{C}$ under an atmosphere of argon. After cooling to room temperature, the mixture was quenched with a saturated aqueous solution of $\mathrm{NaHCO}_{3}$ and extracted with dichloromethane $(3 \times 30 \mathrm{~mL}$ per mmol). The combined organic layers were dried over anhydrous $\mathrm{MgSO}_{4}$. After filtration, the solvent was removed under reduced pressure. The residue was purified by flash column chromatography (eluent: dichloromethane/methanol $100: 0 \rightarrow 93: 7 \mathrm{v} / \mathrm{v}$ ).

\section{Precursor 4-7}

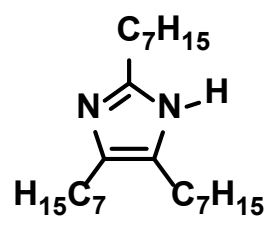

Following the general method, compound 4-7 was synthesized from 1,2-diketone 3-7 (2.0 mmol) and 1-octanal (1-7). Drying under reduced pressure at room temperature afforded the desired product as a yellowish oil. Yield: $60 \%(0.442 \mathrm{~g}) . \delta_{\mathrm{H}}\left(400 \mathrm{MHz}, \mathrm{CDCl}_{3}\right): 0.75-0.95\left(\mathrm{~m}, 9 \mathrm{H}, \mathrm{CH}_{2}-\right.$ $\left.\mathrm{CH}_{3}\right), 1.07-1.46(\mathrm{~m}, 24 \mathrm{H}), 1.48-1.73\left(\mathrm{~m}, 6 \mathrm{H}\right.$, (imidazole C-2/C-4/C-5)-CH$\left.-\mathrm{CH}_{2}\right), 2.46(\mathrm{t}, 4 \mathrm{H}$, (imidazole C-4/C-5)- $\mathrm{CH}_{2}, J=7.7 \mathrm{~Hz}$ ), 2.59 (t, 2H, (imidazole C-2)- $\mathrm{CH}_{2}, J=7.9 \mathrm{~Hz}$ ), 7.6 (broad 
s, $1 \mathrm{H}, \mathrm{N}-\mathrm{H})$. Signals from impurities that could not be removed were observed at $\sim 2.27 \mathrm{ppm}$ and 3.64 ppm. $\delta_{\mathrm{C}}\left(101 \mathrm{MHz}, \mathrm{CDCl}_{3}\right): 14.2,14.2,22.8,22.8,22.8,25.1,27.7,28.7,29.1,29.2,29.4$, 29.5, 29.5, 30.2, 31.9, 32.0, 32.0, 70.7, 146.0. HRMS (ESI, $\mathrm{CH}_{3} \mathrm{OH}, \mathrm{m} / z$ ): calcd. for $\left[\mathrm{C}_{24} \mathrm{H}_{47} \mathrm{~N}_{2}\right]^{+}$ $\left([\mathrm{M}+\mathrm{H}]^{+}\right): 363.3734$; found: 363.3741. $\mathrm{R}_{\mathrm{f}}\left(\mathrm{CH}_{2} \mathrm{Cl}_{2} / \mathrm{CH}_{3} \mathrm{OH} 9: 1 \mathrm{v} / \mathrm{v}\right): 0.13$.

Precursor 4-11

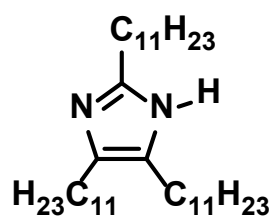

Following the general method, compound 4-11 was synthesized from 1,2-diketone 3-11 (1.4 mmol) and 1-dodecanal (1-11). Drying under reduced pressure at room temperature afforded the desired product as a yellowish oil. Yield: $69 \%(0.515 \mathrm{~g}) . \delta_{\mathrm{H}}\left(300 \mathrm{MHz}, \mathrm{CDCl}_{3}\right): 0.87\left(\mathrm{t}, 9 \mathrm{H}, \mathrm{CH}_{2}-\right.$ $\left.\mathrm{CH}_{3}, J=6.6 \mathrm{~Hz}\right), 1.09-1.38(\mathrm{~m}, 48 \mathrm{H}), 1.47-1.79\left(\mathrm{~m}, 6 \mathrm{H}\right.$, (imidazole C-2/C-4/C-5)-CH $-\mathrm{CH}_{2}$ ), 2.48 (t, 4H, (imidazole C-4/C-5)- $\mathrm{CH}_{2}, J=7.6 \mathrm{~Hz}$ ), 2.71 (t, 2H, (imidazole C-2)- $\mathrm{CH}_{2}, J=7.8 \mathrm{~Hz}$ ), $\sim 10.6$ (broad s, 1H, N-H). $\delta_{\mathrm{C}}\left(75 \mathrm{MHz}, \mathrm{CDCl}_{3}\right): 22.8,24.8,27.3,28.6,29.4,29.5,29.5,29.6$, 29.8, 29.8, 29.9, 30.0, 32.1, 145.9. HRMS (ESI, $\left.\mathrm{CH}_{3} \mathrm{OH}, m / z\right)$ : calcd. for $\left[\mathrm{C}_{36} \mathrm{H}_{71} \mathrm{~N}_{2}\right]^{+}\left([\mathrm{M}+\mathrm{H}]^{+}\right)$: 531.5612; found: 531.5614. $\mathrm{R}_{\mathrm{f}}\left(\mathrm{CH}_{2} \mathrm{Cl}_{2} / \mathrm{CH}_{3} \mathrm{OH} 9\right.$ : $\left.1 \mathrm{v} / \mathrm{v}\right): 0.37$.

Precursor 4-15

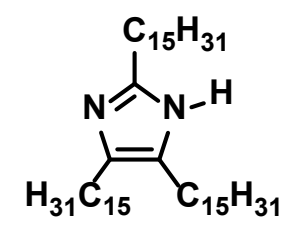

Following the general method, compound 4-15 was synthesized from 1,2-diketone 3-15 (2.1 mmol) and 1-hexadecanal (1-15). Drying under reduced pressure at room temperature afforded the desired product as a yellowish oil. Yield: $29 \%(0.425 \mathrm{~g}) . \delta_{\mathrm{H}}\left(300 \mathrm{MHz}, \mathrm{CDCl}_{3}\right): 0.84-0.90$ (m, 9H, $\left.\mathrm{CH}_{2}-\mathrm{CH}_{3}\right), 1.19-1.31$ (m, 72H), 1.51-1.71 (m, 6H, (imidazole C-2/C-4/C-5)- $\mathrm{CH}_{2}-\mathrm{CH}_{2}$ ), 2.47 (t, 4H, (imidazole C-4/C-5)- $\mathrm{CH}_{2}, J=7.7 \mathrm{~Hz}$ ), 2.65 (t, 2H, (imidazole C-2)- $\mathrm{CH}_{2}, J=8.0 \mathrm{~Hz}$ ), 
6.6 (broad s, 1H, N-H). $\delta_{\mathrm{C}}\left(75 \mathrm{MHz}, \mathrm{CDCl}_{3}\right): 14.3,22.8,24.8,27.3,28.6,29.4,29.5,29.5,29.5$, 29.6, 29.8, 29.8, 29.9, 30.1, 32.1, 145.9. HRMS (ESI, $\left.\mathrm{CH}_{3} \mathrm{OH}, \mathrm{m} / z\right)$ : calcd. for $\left[\mathrm{C}_{48} \mathrm{H}_{95} \mathrm{~N}_{2}\right]^{+}([\mathrm{M}+$ $\left.\mathrm{H}]^{+}\right)$: 699.7490; found: 699.7490. $\mathrm{R}_{\mathrm{f}}\left(\mathrm{CH}_{2} \mathrm{Cl}_{2} / \mathrm{CH}_{3} \mathrm{OH} 9\right.$ : $\left.1 \mathrm{v} / \mathrm{v}\right): 0.34$.

\section{Precursor $\mathrm{Na}\left[\mathrm{Cp}(\mathrm{CN})_{5}\right]$}

Sodium pentacyanocyclopentadienide was synthesized from $\mathrm{NaCN}$ and $\mathrm{CS}_{2}$ in four steps (see Scheme S1) as described elsewhere. ${ }^{6}$

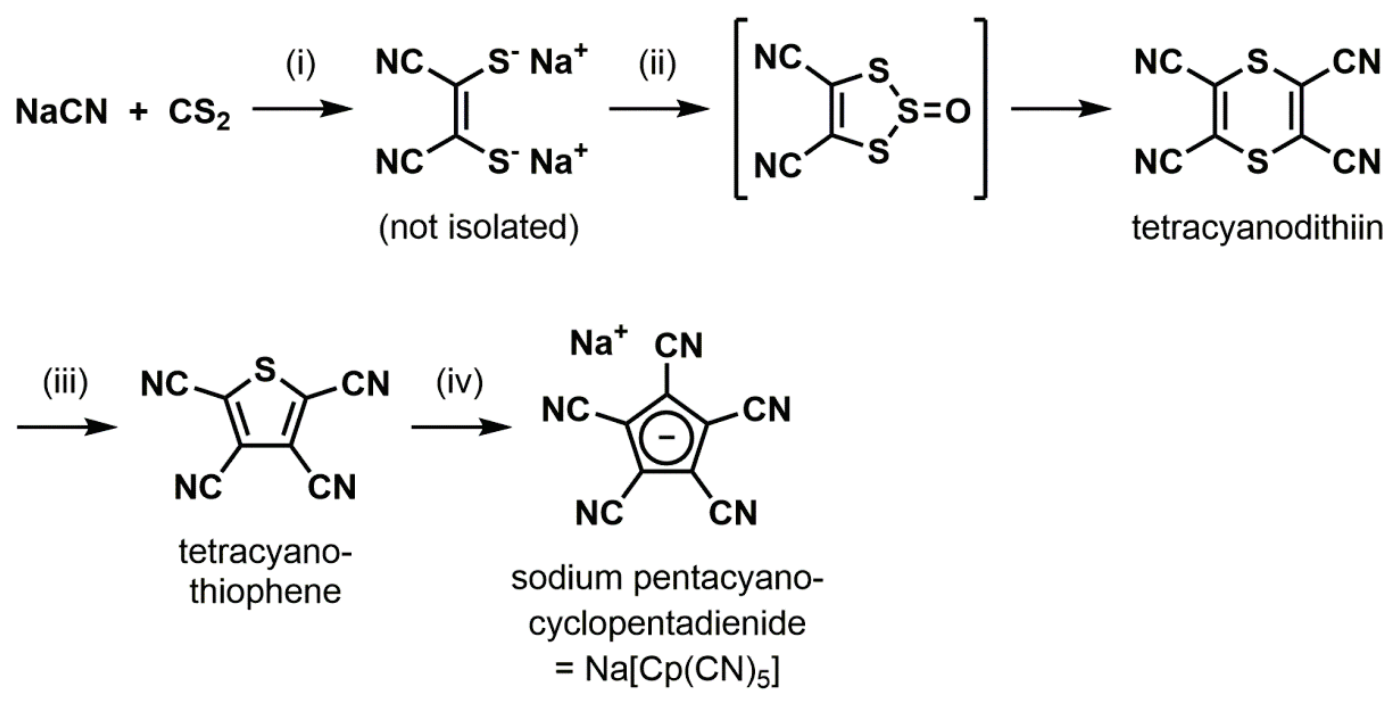

Scheme S1. Synthesis of $\mathrm{Na}\left[\mathrm{Cp}(\mathrm{CN})_{5}\right]$. Reagents and conditions: (i) 1. DMF, r.t. (3 h); 2. $\mathrm{H}_{2} \mathrm{O}$, r.t. (12 h); (ii) $\left(\mathrm{NH}_{4}\right)_{2} \mathrm{~S}_{2} \mathrm{O}_{8}, \mathrm{H}_{2} \mathrm{O}$, r.t. $\left(15\right.$ min.) (yield: $78 \%$ (lit. $\left.75 \%{ }^{6}\right)$ ); (iii) dry and degassed 1,2-dichlorobenzene, $200{ }^{\circ} \mathrm{C}, \mathrm{N}_{2}(2 \mathrm{~h}$ ) (yield: $74 \%$ (lit. $\left.79 \%{ }^{6}\right)$ ); (iv) 1. $p$-toluenesulfonylacetonitrile, $\mathrm{NaH}\left(60 \%\right.$ dispersion in mineral oil), dry THF, $0{ }^{\circ} \mathrm{C}$, $\mathrm{N}_{2}$ (30 min.); 2. dry THF, $-40{ }^{\circ} \mathrm{C}, \mathrm{N}_{2}(1 \mathrm{~h})$ (yield: $95 \%$ (lit. $\left.96 \%{ }^{6}\right)$ ). 


\title{
1.2.2. Syntheses of iodide salt 5-I-1 and bromide salts 5-Br- $n(n=7,11,15)$
}

\author{
Compound 5-I-1 $\mathbf{1}^{7}$
}

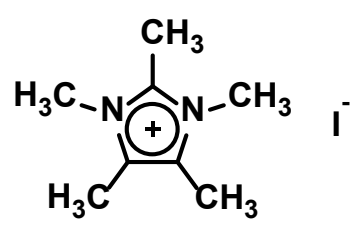

Compound 5-I-1 was synthesized by slowly adding iodomethane (80.32 mmol, $5.0 \mathrm{~mL})$ to a stirred solution of 1,2,4,5-tetramethyl-1H-imidazole $(40.27 \mathrm{mmol}, 5.00 \mathrm{~g})$ in $15 \mathrm{~mL}$ of dry acetonitrile in an oven-dried Schlenk pressure tube. The reaction mixture was covered from light and stirred for $18 \mathrm{~h}$ at $80{ }^{\circ} \mathrm{C}$ under an atmosphere of nitrogen. The solvent and excess of iodomethane were removed under reduced pressure. The crude product was washed with diethyl ether $(3 \times 40 \mathrm{~mL})$ and recrystallized from acetone. Drying under reduced pressure at $50{ }^{\circ} \mathrm{C}$ afforded the desired product as a white powder. Yield: $95 \%(10.16 \mathrm{~g}) . \delta_{\mathrm{H}}\left(400 \mathrm{MHz}, \mathrm{CD}_{2} \mathrm{Cl}_{2}\right)$ : $2.24\left(\mathrm{~s}, 6 \mathrm{H}\right.$, (imidazolium C-4/C-5)- $\left.\mathrm{CH}_{3}\right), 2.74$ (s, 3H, (imidazolium C-2)- $\mathrm{CH}_{3}$ ), 3.70 (s, 6H, N$\left.\mathrm{CH}_{3}\right) . \delta_{\mathrm{C}}\left(101 \mathrm{MHz}, \mathrm{CDCl}_{3}\right)$ : 9.1, 12.7, 33.2, 125.7, 142.8. IR (Ge-ATR): see Section 1.2.7. HRMS (ESI, $\left.\mathrm{CH}_{2} \mathrm{Cl}_{2}, \mathrm{~m} / z\right)$ : calcd. for $\left[\mathrm{C}_{8} \mathrm{H}_{15} \mathrm{~N}_{2}\right]^{+}\left(\left[\mathrm{M}-\mathrm{I}^{-}\right]^{+}\right)$: 139.1230; found: 139.1233. Calcd. for $\mathrm{C}_{8} \mathrm{H}_{15} \mathrm{IN}_{2}$ (266.13): C 36.11, H 5.68, N 10.53. Found: C 36.00, H 5.70, N 10.86.

General method for the synthesis of bromide salts $\mathbf{5 - B r}-\boldsymbol{n}(n=7,11,15)$

The appropriate 2,4,5-trialkyl-1H-imidazole 4-n (1 equiv.), the appropriate 1-bromoalkane $\mathrm{C}_{n} \mathrm{H}_{2 n+1} \mathrm{Br}$ (15 equiv.) and $\mathrm{K}_{2} \mathrm{CO}_{3}$ (2 equiv.) were dissolved in dry tetrahydrofuran ([2,4,5trialkyl- $1 H$-imidazole] $=0.3 \mathrm{M}$ ) in a flame-dried Schlenk pressure tube. The reaction mixture was stirred for 3 days at $80{ }^{\circ} \mathrm{C}$ under an atmosphere of argon. After cooling to room temperature, the mixture was diluted with water. The resulting biphasic system was extracted with dichloromethane $(3 \times 20 \mathrm{~mL}$ per $\mathrm{mmol})$. The combined organic layers were dried over anhydrous $\mathrm{MgSO}_{4}$. After filtration, the solvent was removed under reduced pressure. The residue was purified by flash column chromatography (eluent: dichloromethane/methanol $100: 0 \rightarrow 93: 7$ $\mathrm{v} / \mathrm{v}$ ). To increase the isolated yield of pure product, the column fractions that contained a mixture 
of the 1,2,4,5-tetraalkyl-1H-imidazole and the 1,2,3,4,5-pentaalkyl-1H-imidazol-3-ium bromide were concentrated and re-subjected to the aforementioned reaction conditions.

\section{Compound 5-Br-7}

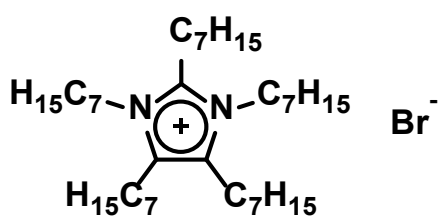

Following the general method, compound 5-Br-7 was synthesized from imidazole 4-7 (1.2 mmol) and 1-bromoheptane. Drying under reduced pressure at room temperature afforded the desired product as a yellow, very viscous oil or wax. Yield: $28 \%(0.217 \mathrm{~g})$. Re-subjecting the column fractions that contained a mixture of the tetra- and pentaalkylated products $(0.88 \mathrm{mmol})$ to the reaction conditions described in the general method provided, after purification, the desired product in $49 \%$ yield $(0.272 \mathrm{~g}) . \delta_{\mathrm{H}}\left(400 \mathrm{MHz}, \mathrm{CDCl}_{3}\right): 0.76-0.96\left(\mathrm{~m}, 15 \mathrm{H}, \mathrm{CH}_{2}-\mathrm{CH}_{3}\right), 1.18-1.57$ (m, 44H), 1.63 (quint, 2H, (imidazolium C-2)- $\mathrm{CH}_{2}-\mathrm{CH}_{2}, J=7.8 \mathrm{~Hz}$ ), 1.73 (quint, 4H, N-CH${ }_{2}$ $\mathrm{CH}_{2}, J=7.7 \mathrm{~Hz}$ ), 2.54 (t, 4H, (imidazolium C-4/C-5)- $\mathrm{CH}_{2}, J=8.1 \mathrm{~Hz}$ ), 3.25 (t, 2H, (imidazolium $\left.\mathrm{C}-2)-\mathrm{CH}_{2}, J=8.1 \mathrm{~Hz}\right), 4.10\left(\mathrm{t}, 4 \mathrm{H}, \mathrm{N}-\mathrm{CH}_{2}, J=8.3 \mathrm{~Hz}\right) . \delta_{\mathrm{C}}\left(101 \mathrm{MHz}, \mathrm{CDCl}_{3}\right)$ : 14.1, 14.1, 22.6, 22.7, 23.4, 25.0, 26.8, 28.6, 28.8, 28.9, 29.0, 29.5, 29.6, 29.8, 31.0, 31.6, 31.7, 46.1 , 70.7, 129.8, 146.1. IR (Ge-ATR): see Section 1.2.7. HRMS (ESI, $\left.\mathrm{CH}_{3} \mathrm{OH}, m / z\right)$ : calcd. for $\left[\mathrm{C}_{38} \mathrm{H}_{75} \mathrm{~N}_{2}\right]^{+}([\mathrm{M}-$ $\left.\left.\mathrm{Br}^{-}\right]^{+}\right)$: 559.5925; found: 559.5919. $\mathrm{R}_{\mathrm{f}}\left(\mathrm{CH}_{2} \mathrm{Cl}_{2} / \mathrm{CH}_{3} \mathrm{OH} 9: 1 \mathrm{v} / \mathrm{v}\right): 0.48$. Calcd. for $\mathrm{C}_{38} \mathrm{H}_{75} \mathrm{BrN}_{2}$ (639.94): C 71.32, H 11.81, N 4.38. Found: C 71.19, H 11.95, N 4.66.

\section{Compound 5-Br-11}

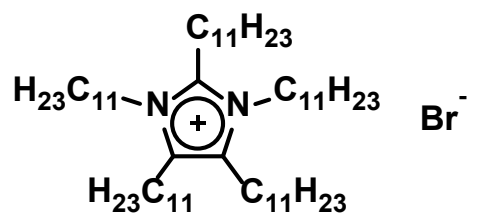

Following the general method, compound 5-Br-11 was synthesized from imidazole 4-11 (0.44 mmol) and 1-bromoundecane. Drying under reduced pressure at room temperature afforded the 
desired product as a slightly sticky, pale-yellowish solid. Yield: 27\% (0.114 g). Re-subjecting the column fractions that contained a mixture of the tetra- and pentaalkylated products $(0.32 \mathrm{mmol})$ to the reaction conditions described in the general method provided, after purification, the desired product in $32 \%$ yield $(0.100 \mathrm{~g}) . \delta_{\mathrm{H}}\left(300 \mathrm{MHz}, \mathrm{CDCl}_{3}\right): 0.85\left(\mathrm{t}, 15 \mathrm{H}, \mathrm{CH}_{2}-\mathrm{CH}_{3}, J=6.8 \mathrm{~Hz}\right), 1.18-$ $1.76(\mathrm{~m}, 90 \mathrm{H}), 2.52$ (t, 4H, (imidazolium C-4/C-5)- $\left.\mathrm{CH}_{2}, J=7.9 \mathrm{~Hz}\right), 3.19$ (t, 2H, (imidazolium $\left.\mathrm{C}-2)-\mathrm{CH}_{2}, J=7.8 \mathrm{~Hz}\right), 4.07\left(\mathrm{t}, 4 \mathrm{H}, \mathrm{N}-\mathrm{CH}_{2}, J=8.1 \mathrm{~Hz}\right) . \delta_{\mathrm{C}}\left(75 \mathrm{MHz}, \mathrm{CDCl}_{3}\right): 14.2,22.8,23.4$, 24.8, 26.1, 26.8, 28.5, 29.2, 29.2, 29.3, 29.4, 29.4, 29.5, 29.5, 29.6, 29.6, 29.7, 29.7, 29.8, 31.0, 32.0, 46.0, 70.6, 129.8, 145.9. IR (Ge-ATR): see Section 1.2.7. HRMS (ESI, $\mathrm{CH}_{3} \mathrm{OH}, \mathrm{m} / \mathrm{z}$ ): calcd. for $\left[\mathrm{C}_{58} \mathrm{H}_{115} \mathrm{~N}_{2}\right]^{+}\left(\left[\mathrm{M}-\mathrm{Br}^{-}\right]^{+}\right): 839.9055$; found: 839.5063. $\mathrm{R}_{\mathrm{f}}\left(\mathrm{CH}_{2} \mathrm{Cl}_{2} / \mathrm{CH}_{3} \mathrm{OH} 9\right.$ : $\left.1 \mathrm{v} / \mathrm{v}\right): 0.41$. Calcd. for $\mathrm{C}_{58} \mathrm{H}_{115} \mathrm{BrN}_{2}$ (920.48): C 75.68, H 12.59, N 3.04. Found: C 75.55, H 12.34, N 3.19.

\section{Compound 5-Br-15}

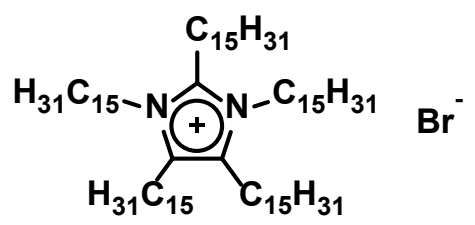

Following the general method, compound 5-Br-15 was synthesized from imidazole 4-15 (0.60 mmol) and 1-bromopentadecane. Drying under reduced pressure at room temperature afforded the desired product as a pale-yellow to pale-brown powder. Yield: $52 \%(0.367 \mathrm{~g}) . \delta_{\mathrm{H}}(400 \mathrm{MHz}$, $\mathrm{CDCl}_{3}$ ): 0.87 (t, 15H, $\left.\mathrm{CH}_{2}-\mathrm{CH}_{3}, J=6.5 \mathrm{~Hz}\right), 1.21-1.79(\mathrm{~m}, 130 \mathrm{H}), 2.54$ (t, 4H, (imidazolium C4/C-5)- $\mathrm{CH}_{2}, J=7.9 \mathrm{~Hz}$ ), 3.26 (t, $2 \mathrm{H}$, (imidazolium C-2)- $\mathrm{CH}_{2}, J=7.9 \mathrm{~Hz}$ ), 4.10 (t, 4H, N-CH $2, J$ = 7.9 Hz). $\delta_{\mathrm{C}}\left(101 \mathrm{MHz}, \mathrm{CDCl}_{3}\right): 14.3,22.8,23.5,25.1,26.9,28.6,29.2,29.3,29.4,29.5,29.6$, 29.6, 29.6, 29.7, 29.8, 29.8, 29.8, 29.8, 29.9, 31.0, 32.0, 46.1, 70.7, 129.8, 146.1. IR (Ge-ATR): see Section 1.2.7. HRMS (ESI, $\left.\mathrm{CH}_{3} \mathrm{OH}, m / z\right)$ : calcd. for $\left[\mathrm{C}_{78} \mathrm{H}_{155} \mathrm{~N}_{2}\right]^{+}\left(\left[\mathrm{M}-\mathrm{Br}^{-}\right]^{+}\right)$: 1120.2185 ; found: 1120.2171. $\mathrm{R}_{\mathrm{f}}\left(\mathrm{CH}_{2} \mathrm{Cl}_{2} / \mathrm{CH}_{3} \mathrm{OH} 9: 1 \mathrm{v} / \mathrm{v}\right): 0.51$. Calcd. for $\mathrm{C}_{78} \mathrm{H}_{155} \mathrm{BrN}_{2}$ (1201.02): C 78.01, H 13.01, N 2.33. Found: C 77.86, H 13.00, N 2.52. 


\subsubsection{Syntheses of nitrate salts $5-\mathrm{NO}_{3}-n(n=1,7,11,15)$}

\section{Compound 5-NO $\mathrm{O}_{3}-1$}

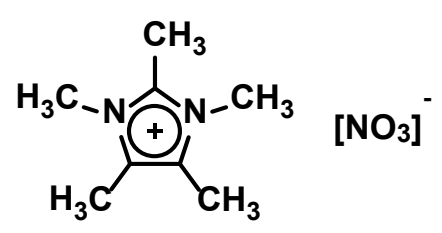

The following steps were performed in the dark. A solution of compound 5-I-1 (3.86 mmol, $1.0279 \mathrm{~g}$ ) in $10 \mathrm{~mL}$ of water was slowly added to a solution of silver nitrate (3.86 mmol, 0.6561 g) in $15 \mathrm{~mL}$ of water under stirring. The reaction mixture was stirred for $1.5 \mathrm{~h}$ at room temperature. Insoluble products were filtered using a paper filter. The filtrate was concentrated under reduced pressure at $40{ }^{\circ} \mathrm{C}$. The residue was re-dissolved in $20 \mathrm{~mL}$ of ethanol and the solution was filtered using a PTFE syringe filter $(0.1 \mu \mathrm{m}$ pore size $)$. After removing the solvent under reduced pressure at $40{ }^{\circ} \mathrm{C}$, the residue was re-dissolved in $10 \mathrm{~mL}$ of dichloromethane and the solution was kept in a freezer overnight. Although visually there were no signs of precipitation, the solution was filtered using a PTFE syringe filter $(0.1 \mu \mathrm{m}$ pore size $)$. Finally, the solution was concentrated and dried under reduced pressure at $60{ }^{\circ} \mathrm{C}$ to afford the desired product as a white powder. Small amounts of the product were dissolved in water and tested with $\mathrm{NaBr}$ or $\mathrm{AgNO}_{3}$; the lack of a precipitate indicated that $\mathrm{AgNO}_{3}$ and the iodide salt, respectively, had been successfully removed. Yield: $95 \%(0.737 \mathrm{~g}) . \delta_{\mathrm{H}}\left(400 \mathrm{MHz}, \mathrm{DMSO}-d_{6}\right): 2.21 \quad(\mathrm{~s}, 6 \mathrm{H}$, (imidazolium C-4/C-5)- $\mathrm{CH}_{3}$ ), 2.57 (s, 3H, (imidazolium C-2)- $\left.\mathrm{CH}_{3}\right), 3.61\left(\mathrm{~s}, 6 \mathrm{H}, \mathrm{N}-\mathrm{CH}_{3}\right) . \delta_{\mathrm{C}}(101$ MHz, DMSO- $\left.d_{6}\right)$ : 7.9, 9.6, 31.5, 124.9, 142.6. IR (Ge-ATR): see Section 1.2.7. HRMS (ESI, $\left.\mathrm{CH}_{3} \mathrm{OH}, \mathrm{m} / \mathrm{z}\right)$ : calcd. for $\left[\mathrm{C}_{8} \mathrm{H}_{15} \mathrm{~N}_{2}\right]^{+}\left(\left[\mathrm{M}-\left[\mathrm{NO}_{3}\right]^{-}\right]^{+}\right)$: 139.1230; found: 139.1228. Calcd. for $\mathrm{C}_{8} \mathrm{H}_{15} \mathrm{~N}_{3} \mathrm{O}_{3} \cdot 0.25 \mathrm{H}_{2} \mathrm{O}$ (205.73): C 47.75, H 7.51, N 20.88 (we note that, upon standing open to air, the compound rapidly absorbed additional water). Found: C 47.42, H 7.62, N 20.45. 
General method for the synthesis of nitrate salts $5-\mathrm{NO}_{3}-\boldsymbol{n}(n=7,11,15)$

The following steps were performed in the dark. A solution of the appropriate bromide salt 5-Br$\boldsymbol{n}$ (1 equiv.) in ethanol $([\mathbf{5}-\mathbf{B r}-\boldsymbol{n}]=0.04 \mathrm{M})$ was slowly added to a solution of silver nitrate (1.1 equiv.) in ethanol $\left(\left[\mathrm{AgNO}_{3}\right]=0.1 \mathrm{M}\right)$ under stirring. The reaction mixture was stirred for $15 \mathrm{~h}$ at room temperature. Subsequently, $6 \mathrm{~mL}$ of dichloromethane was added. Insoluble products were filtered using a paper filter. The filtrate was concentrated under reduced pressure at $40{ }^{\circ} \mathrm{C}$. The residue was re-dissolved in $20 \mathrm{~mL}$ of dichloromethane and washed two times with $5 \mathrm{~mL}$ of water. The organic layer was dried over anhydrous $\mathrm{MgSO}_{4}$. After filtration, the solvent of the filtrate was removed under reduced pressure.

\section{Compound 5- $\mathrm{NO}_{3^{-}}-7$}

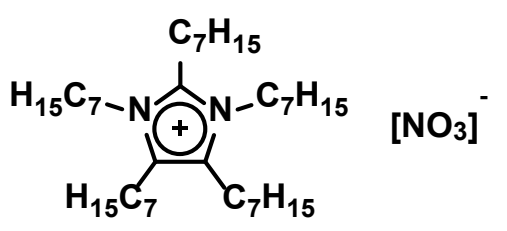

Following the general method, compound 5-NO $\mathrm{NO}_{3}-\mathbf{7}$ was synthesized from bromide salt $5-\mathrm{Br}-\mathbf{7}$ (0.099 mmol) and $\mathrm{AgNO}_{3}$. Drying under reduced pressure at $50{ }^{\circ} \mathrm{C}$ afforded the desired product as an orange-brown, viscous liquid. Small amounts of the product were dissolved in water/ethanol $(1: 1 \mathrm{v} / \mathrm{v})$ and tested with $\mathrm{NaBr}$ or $\mathrm{AgNO}_{3}$; the lack of a precipitate indicated that the slight excess of $\mathrm{AgNO}_{3}$ and the bromide salt, respectively, had been successfully removed. Yield: $78 \%(0.048 \mathrm{~g}) . \delta_{\mathrm{H}}\left(400 \mathrm{MHz}, \mathrm{CDCl}_{3}\right): 0.86-0.91\left(\mathrm{~m}, 15 \mathrm{H}, \mathrm{CH}_{2}-\mathrm{CH}_{3}\right), 1.22-1.58(\mathrm{~m}$, 44H), 1.63 (quint, $2 \mathrm{H}$, (imidazolium C-2)- $\mathrm{CH}_{2}-\mathrm{CH}_{2}, J=7.8 \mathrm{~Hz}$ ), 1.73 (quint, $4 \mathrm{H}, \mathrm{N}-\mathrm{CH}_{2}-\mathrm{CH}_{2}, J$ $=7.7 \mathrm{~Hz}$ ), 2.55 (t, 4H, (imidazolium C-4/C-5)- $\mathrm{CH}_{2}, J=8.1 \mathrm{~Hz}$ ), 3.09 (t, 2H, (imidazolium C-2)$\left.\mathrm{CH}_{2}, J=8.3 \mathrm{~Hz}\right), 4.03\left(\mathrm{t}, 4 \mathrm{H}, \mathrm{N}-\mathrm{CH}_{2}, J=8.3 \mathrm{~Hz}\right) . \delta_{\mathrm{C}}\left(101 \mathrm{MHz}, \mathrm{CDCl}_{3}\right): 14.1,14.2,22.6,22.7$, 22.7, 23.4, 24.5, 26.9, 28.4, 28.8, 28.9, 29.6, 29.7, 29.7, 30.9, 31.7, 31.8, 45.9, 70.7, 129.9, 145.7. IR (Ge-ATR): see Section 1.2.7. HRMS (ESI, $\left.\mathrm{CH}_{2} \mathrm{Cl}_{2}, \mathrm{~m} / z\right)$ : calcd. for $\left[\mathrm{C}_{38} \mathrm{H}_{75} \mathrm{~N}_{2}\right]^{+}([\mathrm{M}-$ $\left.\left[\mathrm{NO}_{3}\right]^{-}\right]^{+}$): 559.5925; found: 559.5955. Calcd. for $\mathrm{C}_{38} \mathrm{H}_{75} \mathrm{~N}_{3} \mathrm{O}_{3} \cdot 0.5 \mathrm{H}_{2} \mathrm{O}$ (631.05): C 72.33, $\mathrm{H}$ 12.14, N 6.66. Found: C 72.36, H 12.17, N 6.24. 


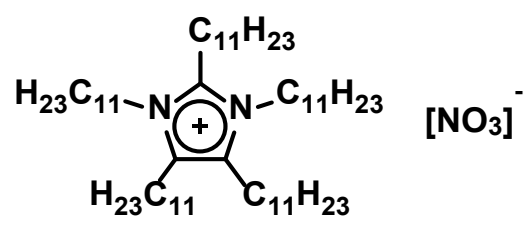

Following the general method, compound 5-NO $\mathrm{NO}_{3}-\mathbf{1 1}$ was synthesized from bromide salt 5-Br-11 (0.099 mmol) and $\mathrm{AgNO}_{3}$. Drying under reduced pressure at $50{ }^{\circ} \mathrm{C}$ afforded the desired product as a slightly sticky, dark-yellow to pale-brown solid. Small amounts of the product were dissolved in water/ethanol $(1: 1 \mathrm{v} / \mathrm{v})$ and tested with $\mathrm{NaBr}$ or $\mathrm{AgNO}_{3}$; the lack of a precipitate indicated that the slight excess of $\mathrm{AgNO}_{3}$ and the bromide salt, respectively, had been successfully removed. Yield: $80 \%(0.071 \mathrm{~g}) . \delta_{\mathrm{H}}\left(400 \mathrm{MHz}, \mathrm{CDCl}_{3}\right): 0.87\left(\mathrm{t}, 15 \mathrm{H}, \mathrm{CH}_{2}-\mathrm{CH}_{3}, J=\right.$ $6.8 \mathrm{~Hz}$ ), 1.20-1.57 (m, 84H), 1.62 (quint, 2H, (imidazolium C-2)- $\mathrm{CH}_{2}-\mathrm{CH}_{2}, J=7.9 \mathrm{~Hz}$ ), 1.72 (quint, $4 \mathrm{H}, \mathrm{N}-\mathrm{CH}_{2}-\mathrm{CH}_{2}, J=7.8 \mathrm{~Hz}$ ), 2.53 (t, 4H, (imidazolium C-4/C-5)- $\mathrm{CH}_{2}, J=8.1 \mathrm{~Hz}$ ), 3.07 (t, 2H, (imidazolium C-2)- $\mathrm{CH}_{2}, J=8.2 \mathrm{~Hz}$ ), $4.01\left(\mathrm{t}, 4 \mathrm{H}, \mathrm{N}-\mathrm{CH}_{2}, J=8.1 \mathrm{~Hz}\right) . \delta_{\mathrm{C}}(101 \mathrm{MHz}$, $\left.\mathrm{CDCl}_{3}\right): 14.2,22.8,23.4,24.3,26.9,28.3,29.2,29.3,29.5,29.5,29.5,29.6,29.6,29.6,29.7$, 29.7, 29.7, 29.8, 30.9, 32.0, 45.8, 70.7, 129.9, 145.7. IR (Ge-ATR): see Section 1.2.7. HRMS (ESI, $\left.\mathrm{CH}_{2} \mathrm{Cl}_{2}, \mathrm{~m} / z\right)$ : calcd. for $\left[\mathrm{C}_{58} \mathrm{H}_{115} \mathrm{~N}_{2}\right]^{+}\left(\left[\mathrm{M}-\left[\mathrm{NO}_{3}\right]^{-}\right]^{+}\right)$: 839.9055; found: 839.9053. Calcd. for $\mathrm{C}_{58} \mathrm{H}_{115} \mathrm{~N}_{3} \mathrm{O}_{3} \cdot 0.5 \mathrm{H}_{2} \mathrm{O}$ (911.59): C 76.42, H 12.83, N 4.61. Found: C 76.43, H 12.99, N 4.29.

\section{Compound 5-NO $\mathrm{O}_{3}-15$}

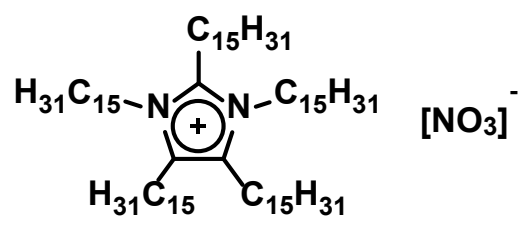

Following the general method, compound 5- $\mathrm{NO}_{3}-15$ was synthesized from bromide salt 5-Br-15 $(0.075 \mathrm{mmol})$ and $\mathrm{AgNO}_{3}$. Drying under reduced pressure at $50{ }^{\circ} \mathrm{C}$ afforded the desired product as a pale-yellow to pale-brown powder. Small amounts of the product were dissolved in water/ethanol $(1: 1 \mathrm{v} / \mathrm{v})$ and tested with $\mathrm{NaBr}$ or $\mathrm{AgNO}_{3}$; the lack of a precipitate indicated that the slight excess of $\mathrm{AgNO}_{3}$ and the bromide salt, respectively, had been successfully removed. 
Yield: $73 \%\left(0.065\right.$ g). $\delta_{\mathrm{H}}\left(400 \mathrm{MHz}, \mathrm{CDCl}_{3}\right): 0.87$ (t, 15H, $\left.\mathrm{CH}_{2}-\mathrm{CH}_{3}, J=6.8 \mathrm{~Hz}\right), 1.19-1.57(\mathrm{~m}$, 124H), 1.62 (quint, 2H, (imidazolium C-2) $-\mathrm{CH}_{2}-\mathrm{CH}_{2}, J=7.9 \mathrm{~Hz}$ ), 1.72 (quint, 4H, N-CH $-\mathrm{CH}_{2}$, $J=7.9 \mathrm{~Hz}$ ), 2.54 (t, 4H, (imidazolium C-4/C-5)- $\mathrm{CH}_{2}, J=8.1 \mathrm{~Hz}$ ), 3.09 (t, 2H, (imidazolium C2) $\left.-\mathrm{CH}_{2}, J=8.2 \mathrm{~Hz}\right), 4.02\left(\mathrm{t}, 4 \mathrm{H}, \mathrm{N}-\mathrm{CH}_{2}, J=8.3 \mathrm{~Hz}\right) . \delta_{\mathrm{C}}\left(101 \mathrm{MHz}, \mathrm{CDCl}_{3}\right): 14.3,22.8,23.4$, 24.4, 26.9, 28.4, 29.2, 29.3, 29.5, 29.6, 29.6, 29.6, 29.7, 29.7, 29.7, 29.8, 29.8, 29.8, 29.8, 29.9, 30.9, 32.1, 45.8, 70.7, 129.9, 145.7. IR (Ge-ATR): see Section 1.2.7. HRMS (ESI, $\left.\mathrm{CH}_{2} \mathrm{Cl}_{2}, \mathrm{~m} / z\right)$ : calcd. for $\left[\mathrm{C}_{78} \mathrm{H}_{155} \mathrm{~N}_{2}\right]^{+}\left(\left[\mathrm{M}-\left[\mathrm{NO}_{3}\right]^{-}\right]^{+}\right):$1120.2185; found: 1120.2164. Calcd. for $\mathrm{C}_{78} \mathrm{H}_{155} \mathrm{~N}_{3} \mathrm{O}_{3} \cdot 0.5 \mathrm{H}_{2} \mathrm{O}$ (1192.13): C 78.59, H 13.19, N 3.52. Found: C 78.27, H 13.04, N 3.31. 


\subsubsection{Syntheses of pentacyanocyclopentadienide salts $5-\mathrm{Cp}(\mathrm{CN})_{5}-n(n=1,7,11,15)$}

\section{Compound 5-Cp(CN) $)_{5-1}$}

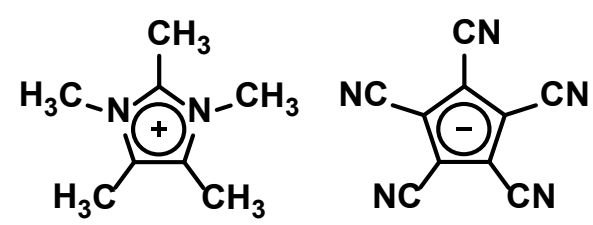

Under stirring, a solution of compound 5-I-1 $(0.353 \mathrm{mmol}, 0.0940 \mathrm{~g})$ in $3 \mathrm{~mL}$ of water was slowly added to a solution of sodium pentacyanocyclopentadienide $(0.316 \mathrm{mmol}, 0.0674 \mathrm{~g})$ in 2 $\mathrm{mL}$ of water. The reaction mixture was stirred for $1 \mathrm{~h}$ at room temperature. The off-white precipitate that had formed was filtered, washed with copious amounts of water, and then dried under reduced pressure at $50{ }^{\circ} \mathrm{C}$ for $48 \mathrm{~h}$. Yield: $77 \%(0.080 \mathrm{~g}) . \delta_{\mathrm{H}}\left(400 \mathrm{MHz}, \mathrm{DMSO}-d_{6}\right): 2.20$ (s, 6H, (imidazolium C-4/C-5)- $\mathrm{CH}_{3}$ ), 2.56 (s, 3H, (imidazolium C-2)- $\mathrm{CH}_{3}$ ), $3.61\left(\mathrm{~s}, 6 \mathrm{H}, \mathrm{N}-\mathrm{CH}_{3}\right.$ ). $\delta_{\mathrm{C}}\left(101 \mathrm{MHz}, \mathrm{DMSO}-d_{6}\right): 8.0,9.7,31.6,101.7\left(-\mathbf{C} \equiv \mathrm{N}\right.$ in $\left.\left[\mathrm{Cp}(\mathrm{CN})_{5}\right]^{-}\right), 113.0\left(\mathrm{C}_{\text {ring }}\right.$ in $\left[\mathrm{Cp}(\mathrm{CN})_{5}\right]^{-}$ ), 125.0, 142.6. IR (Ge-ATR): see Section 1.2.7. HRMS (ESI, DMSO, $m / z$ ): calcd. for $\left[\mathrm{C}_{8} \mathrm{H}_{15} \mathrm{~N}_{2}\right]^{+}$ $\left(\left[\mathrm{M}-\left[\mathrm{Cp}(\mathrm{CN})_{5}\right]^{-}\right]^{+}\right): 139.1230$; found: 139.1226. Calcd. for $\mathrm{C}_{18} \mathrm{H}_{15} \mathrm{~N}_{7}$ (329.36): C 65.64, H 4.59, N 29.77. Found: C 65.26, H 4.54, N 29.40.

General method for the synthesis of pentacyanocyclopentadienide salts $\mathbf{5 - C p}(\mathbf{C N})_{5}-\boldsymbol{n}(n=7,11$, 15)

Under stirring, a solution of the appropriate bromide salt 5-Br-n (1 equiv.) in dry acetonitrile ([5$\mathbf{B r}-\boldsymbol{n}]=0.03 \mathrm{M}$ ) was slowly added to a solution of sodium pentacyanocyclopentadienide (1 equiv. $)$ in dry acetonitrile $\left(\left[\mathrm{Na}\left[\mathrm{Cp}(\mathrm{CN})_{5}\right]\right]=0.07 \mathrm{M}\right)$. The reaction mixture was stirred for $1 \mathrm{~h}$ at room temperature (in the case of 5-Cp(CN) $)_{5} \mathbf{7}$ and $\mathbf{5 - C p}(\mathbf{C N})_{5}-11$ ) or at $65-70{ }^{\circ} \mathrm{C}$ (in the case of 5-Cp(CN)5-15). Subsequently, $30 \mathrm{~mL}$ of dichloromethane was added and the mixture was washed with $10 \mathrm{~mL}$ of water. The organic layer was separated and concentrated under reduced pressure at $40{ }^{\circ} \mathrm{C}$. The residue was further dried overnight under reduced pressure at $40{ }^{\circ} \mathrm{C}$, after which it was re-dissolved in $2.5 \mathrm{~mL}$ of dichloromethane. The solution was kept in a freezer overnight. Although visually there were no signs of precipitation, the solution was filtered using a 
PTFE syringe filter $(0.1 \mu \mathrm{m}$ pore size $)$. Finally the solution was concentrated and dried under reduced pressure at $50{ }^{\circ} \mathrm{C}$.

Compound 5-Cp(CN) $)_{5^{-7}}$
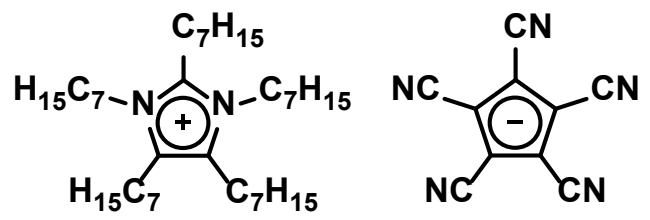

Following the general method, compound 5-Cp(CN) $)_{5}^{-7}$ was synthesized from bromide salt 5-Br$7(0.133 \mathrm{mmol})$ and $\mathrm{Na}\left[\mathrm{Cp}(\mathrm{CN})_{5}\right]$. Drying under reduced pressure at $50{ }^{\circ} \mathrm{C}$ afforded the desired product as a dark-orange liquid. Yield: $99 \%(0.098 \mathrm{~g}) . \delta_{\mathrm{H}}\left(400 \mathrm{MHz}, \mathrm{CDCl}_{3}\right): 0.88\left(\mathrm{t}, 15 \mathrm{H}, \mathrm{CH}_{2^{-}}\right.$ $\mathrm{CH}_{3}, J=6.7 \mathrm{~Hz}$ ), 1.23-1.58 (m, 44H), 1.66 (quint, 2H, (imidazolium C-2)- $\mathrm{CH}_{2}-\mathrm{CH}_{2}, J=8.1$ $\mathrm{Hz}$ ), 1.73 (quint, 4H, N-CH $2-\mathrm{CH}_{2}, J=7.6 \mathrm{~Hz}$ ), 2.57 (t, 4H, (imidazolium C-4/C-5)- $\mathrm{CH}_{2}, J=8.1$ $\mathrm{Hz}$ ), 2.88 (t, 2H, (imidazolium C-2)- $\mathrm{CH}_{2}, J=8.3 \mathrm{~Hz}$ ), 3.94 (t, 4H, N-CH $\left.2, J=8.2 \mathrm{~Hz}\right) . \delta_{\mathrm{C}}(101$ $\mathrm{MHz}_{\mathrm{CDCl}}$ ): 14.1, 14.1, 22.6, 22.6, 22.7, 23.4, 24.4, 26.8, 28.2, 28.7, 28.9, 29.6, 29.8, 30.9, 31.6, 31.6, 31.7, 45.9, $102.6\left(-\mathrm{C} \equiv \mathrm{N}\right.$ in $\left.\left[\mathrm{Cp}(\mathrm{CN})_{5}\right]^{-}\right), 113.6\left(\mathrm{C}_{\text {ring }}\right.$ in $\left.\left[\mathrm{Cp}(\mathrm{CN})_{5}\right]^{-}\right), 130.4,144.6$. IR (Ge-ATR): see Section 1.2.7. HRMS (ESI, $\left.\mathrm{CH}_{2} \mathrm{Cl}_{2}, m / z\right)$ : calcd. for $\left[\mathrm{C}_{38} \mathrm{H}_{75} \mathrm{~N}_{2}\right]^{+}([\mathrm{M}-$ $\left.\left.\left[\mathrm{Cp}(\mathrm{CN})_{5}\right]^{-}\right]^{+}\right): 559.5925$; found: 559.5928. Calcd. for $\mathrm{C}_{48} \mathrm{H}_{75} \mathrm{~N}_{7} \cdot 0.5 \mathrm{H}_{2} \mathrm{O}$ (759.19): C 75.94, $\mathrm{H}$ 10.09, N 12.92. Found: C 75.70, H 9.88, N 12.52.

\section{Compound 5-Cp(CN) $)_{5-11}$}
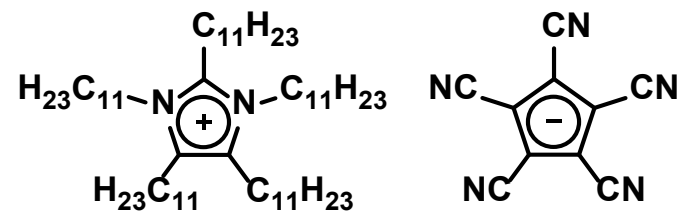

Following the general method, compound 5-Cp(CN) $)_{5}-11$ was synthesized from bromide salt 5Br-11 $(0.100 \mathrm{mmol})$ and $\mathrm{Na}\left[\mathrm{Cp}(\mathrm{CN})_{5}\right]$. Drying under reduced pressure at $50{ }^{\circ} \mathrm{C}$ afforded the desired product as a dark-yellow to dark-orange liquid. Yield: $98 \%(0.101 \mathrm{~g}) . \delta_{\mathrm{H}}(400 \mathrm{MHz}$, $\mathrm{CDCl}_{3}$ ): $0.87\left(\mathrm{t}, 15 \mathrm{H}, \mathrm{CH}_{2}-\mathrm{CH}_{3}, J=6.8 \mathrm{~Hz}\right.$ ), 1.19-1.57 (m, 84H), 1.65 (quint, 2H, (imidazolium 
C-2)- $\mathrm{CH}_{2}-\mathrm{CH}_{2}, J=8.1 \mathrm{~Hz}$ ), 1.73 (quint, $4 \mathrm{H}, \mathrm{N}-\mathrm{CH}_{2}-\mathrm{CH}_{2}, J=7.7 \mathrm{~Hz}$ ), 2.56 (t, 4H, (imidazolium $\mathrm{C}-4 / \mathrm{C}-5$ )- $\mathrm{CH}_{2}, J=8.1 \mathrm{~Hz}$ ), 2.88 (t, 2H, (imidazolium C-2)- $\mathrm{CH}_{2}, J=8.3 \mathrm{~Hz}$ ), 3.94 (t, 4H, N-CH , $J=8.2 \mathrm{~Hz}) . \delta_{\mathrm{C}}\left(101 \mathrm{MHz}, \mathrm{CDCl}_{3}\right): 14.2,22.8,23.4,24.4,26.8,28.2,29.1,29.1,29.3,29.4,29.5$, $29.5,29.5,29.6,29.6,29.7,29.7,29.7,29.8,29.8,30.9,32.0,32.0,45.9,102.6(-\mathbf{C} \equiv \mathrm{N}$ in $\left.\left[\mathrm{Cp}(\mathrm{CN})_{5}\right]^{-}\right)$, $113.6\left(\mathrm{C}_{\text {ring }}\right.$ in $\left.\left[\mathrm{Cp}(\mathrm{CN})_{5}\right]^{-}\right), 130.4,144.6$. IR (Ge-ATR): see Section 1.2.7. HRMS (ESI, $\left.\mathrm{CH}_{2} \mathrm{Cl}_{2}, m / z\right)$ : calcd. for $\left[\mathrm{C}_{58} \mathrm{H}_{115} \mathrm{~N}_{2}\right]^{+}\left(\left[\mathrm{M}-\left[\mathrm{Cp}(\mathrm{CN})_{5}\right]^{-}\right]^{+}\right)$: 839.9055; found: 839.9053 . Calcd. for $\mathrm{C}_{68} \mathrm{H}_{115} \mathrm{~N}_{7} \cdot 0.5 \mathrm{H}_{2} \mathrm{O}$ (1039.73): C 78.55, H 11.25, N 9.43. Found: C 78.55, H 11.40, N 9.16 .

Compound 5-Cp(CN) $)^{-15}$

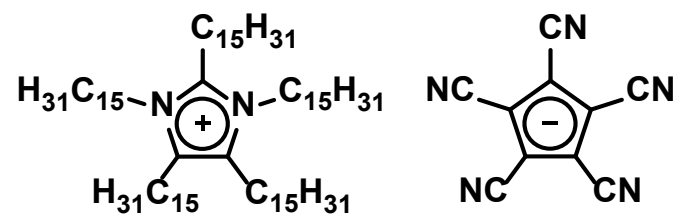

Following the general method, compound 5-Cp(CN) $)_{5}-15$ was synthesized from bromide salt 5Br-15 $(0.075 \mathrm{mmol})$ and $\mathrm{Na}\left[\mathrm{Cp}(\mathrm{CN})_{5}\right]$. Drying under reduced pressure at $50{ }^{\circ} \mathrm{C}$ afforded the desired product as a dark-yellow to dark-orange liquid. Yield: $99 \%(0.097 \mathrm{~g}) . \delta_{\mathrm{H}}(400 \mathrm{MHz}$, $\left.\mathrm{CDCl}_{3}\right): 0.88\left(\mathrm{t}, 15 \mathrm{H}, \mathrm{CH}_{2}-\mathrm{CH}_{3}, J=6.8 \mathrm{~Hz}\right), 1.18-1.78(\mathrm{~m}, 130 \mathrm{H}), 2.56(\mathrm{t}, 4 \mathrm{H}$, (imidazolium C4/C-5)- $\mathrm{CH}_{2}, J=8.1 \mathrm{~Hz}$ ), 2.88 (t, 2H, (imidazolium C-2)- $\mathrm{CH}_{2}, J=8.3 \mathrm{~Hz}$ ), 3.93 (t, 4H, N-CH,$J$ $=8.2 \mathrm{~Hz}) . \delta_{\mathrm{C}}\left(101 \mathrm{MHz}, \mathrm{CDCl}_{3}\right): 14.3,22.8,23.4,24.4,26.8,28.2,29.1,29.1,29.3,29.5,29.5$, $29.6,29.7,29.7,29.8,29.8,29.8,29.9,30.9,32.1,45.9,102.6\left(-\mathrm{C} \equiv \mathrm{N}\right.$ in $\left.\left[\mathrm{Cp}(\mathrm{CN})_{5}\right]\right), 113.6\left(\mathrm{C}_{\text {ring }}\right.$ in $\left.\left[\mathrm{Cp}(\mathrm{CN})_{5}\right]^{-}\right), 130.4,144.6$. IR (Ge-ATR): see Section 1.2.7. HRMS (ESI, $\left.\mathrm{CH}_{2} \mathrm{Cl}_{2}, \mathrm{~m} / z\right)$ : calcd. for $\left[\mathrm{C}_{78} \mathrm{H}_{155} \mathrm{~N}_{2}\right]^{+}\left(\left[\mathrm{M}-\left[\mathrm{Cp}(\mathrm{CN})_{5}\right]^{+}\right]^{+}\right): 1120.2185$; found: 1120.2162. Calcd. for $\mathrm{C}_{88} \mathrm{H}_{155} \mathrm{~N}_{7}$ (1311.26): C 80.61, H 11.92, N 7.48. Found: C 80.25, H 12.17, N 7.20. 


\subsubsection{Summary of NMR data}

The ${ }^{1} \mathrm{H}$ and ${ }^{13} \mathrm{C}$ NMR chemical shifts (recorded in $\mathrm{CDCl}_{3}$ solution) that are listed in Table $\mathrm{S} 1$ show how the $\delta_{\mathrm{C}}$ values for the imidazolium C-2 carbon atoms and the $\delta_{\mathrm{H}}$ values for the methylene groups that are directly connected to the imidazolium C-2 carbon and imidazolium nitrogen atoms are influenced by the nature of the negative counterions. The data suggested to us that the anions are in close proximity to the " $\mathrm{N}-\left[(\mathrm{C}-2)-\left(\mathrm{CH}_{2}\right)-\ldots\right]-\mathrm{N}$ " segments of the imidazolium cations in $\mathrm{CDCl}_{3}$ solution. Similar observations have been reported before for 1,3dialkylimidazolium salts with different anions; see, for example, ref. 8.

Table S1. ${ }^{1} \mathrm{H}$ NMR chemical shifts of the methylene groups that are directly connected to the imidazolium rings and ${ }^{13} \mathrm{C}$ NMR chemical shifts of the imidazolium ring carbon atoms for the series 5-Br-n, 5-NO $\mathbf{N O}_{3}-\boldsymbol{n}$ and $\mathbf{5 - C p}(\mathbf{C N})_{5}-\boldsymbol{n}(n=7,11,15)$ (all of the values were recorded in $\left.\mathrm{CDCl}_{3}\right)^{a}$

\begin{tabular}{|c|c|c|c|c|c|}
\hline Compound & $\begin{array}{c}\delta_{\mathrm{H}} \text { (imidazolium } \\
\mathrm{C}-4 / \mathrm{C}-5)-\mathrm{C} \underline{H}_{2} \\
(\mathrm{ppm})\end{array}$ & $\begin{array}{c}\delta_{\mathrm{H}}(\text { imidazolium } \\
\mathrm{C}-2)-\mathrm{C} \underline{\mathrm{H}}_{2} \\
(\mathrm{ppm})\end{array}$ & $\begin{array}{c}\boldsymbol{\delta}_{\mathrm{H}} \mathrm{N}-\mathrm{C} \underline{\mathrm{H}}_{2} \\
(\mathrm{ppm})\end{array}$ & $\begin{array}{c}\delta_{\mathrm{C}} \text { imidazo- } \\
\underset{(\mathrm{ppm})}{\operatorname{lium} \underline{\mathrm{C}-4} / \underline{\mathrm{C}-5}}\end{array}$ & $\begin{array}{c}\delta_{\mathrm{C}} \text { imidazo- } \\
\underset{(\mathrm{ppm})}{\operatorname{lium} \underline{\mathrm{C}-2}}\end{array}$ \\
\hline $5-\mathrm{Br}-7$ & 2.54 & 3.25 & 4.10 & 129.8 & 146.1 \\
\hline $5-\mathrm{NO}_{3}-7$ & 2.55 & 3.09 & 4.03 & 129.9 & 145.7 \\
\hline 5-Cp(CN) $)_{5}^{-7}$ & 2.57 & 2.88 & 3.94 & 130.4 & 144.6 \\
\hline 5-Br-11 & 2.52 & 3.19 & 4.07 & 129.8 & 145.9 \\
\hline $5-\mathrm{NO}_{3}-11$ & 2.53 & 3.07 & 4.01 & 129.9 & 145.7 \\
\hline $5-\mathrm{Cp}(\mathrm{CN})_{5}-11$ & 2.56 & 2.88 & 3.94 & 130.4 & 144.6 \\
\hline 5-Br-15 & 2.54 & 3.26 & 4.10 & 129.8 & 146.1 \\
\hline $5-\mathrm{NO}_{3}-15$ & 2.54 & 3.09 & 4.02 & 129.9 & 145.7 \\
\hline 5-Cp $(\mathrm{CN})_{5}-15$ & 2.56 & 2.88 & 3.93 & 130.4 & 144.6 \\
\hline
\end{tabular}

We note that the ${ }^{13} \mathrm{C}$ chemical shifts (recorded in $\mathrm{CDCl}_{3}$ solution) of the imidazole $\mathrm{C}-2$ carbon atoms in precursors 4- $\boldsymbol{n}(n=7,11,15)$ (i.e., $146.0 \mathrm{ppm}, 145.9 \mathrm{ppm}$ and $145.9 \mathrm{ppm}$, respectively) did not change significantly upon alkylation of the nitrogen atoms to form compounds $\mathbf{5}-\mathbf{B r}-\boldsymbol{n}(\boldsymbol{n}$ $=7,11,15)$ (see Table $\mathrm{S} 1$ for the values in ppm). 


\subsubsection{NMR spectra}

Compound 4-7 ( $\left.{ }^{l} \mathrm{H}, 400 \mathrm{MHz}, \mathrm{CDCl}_{3}\right)$

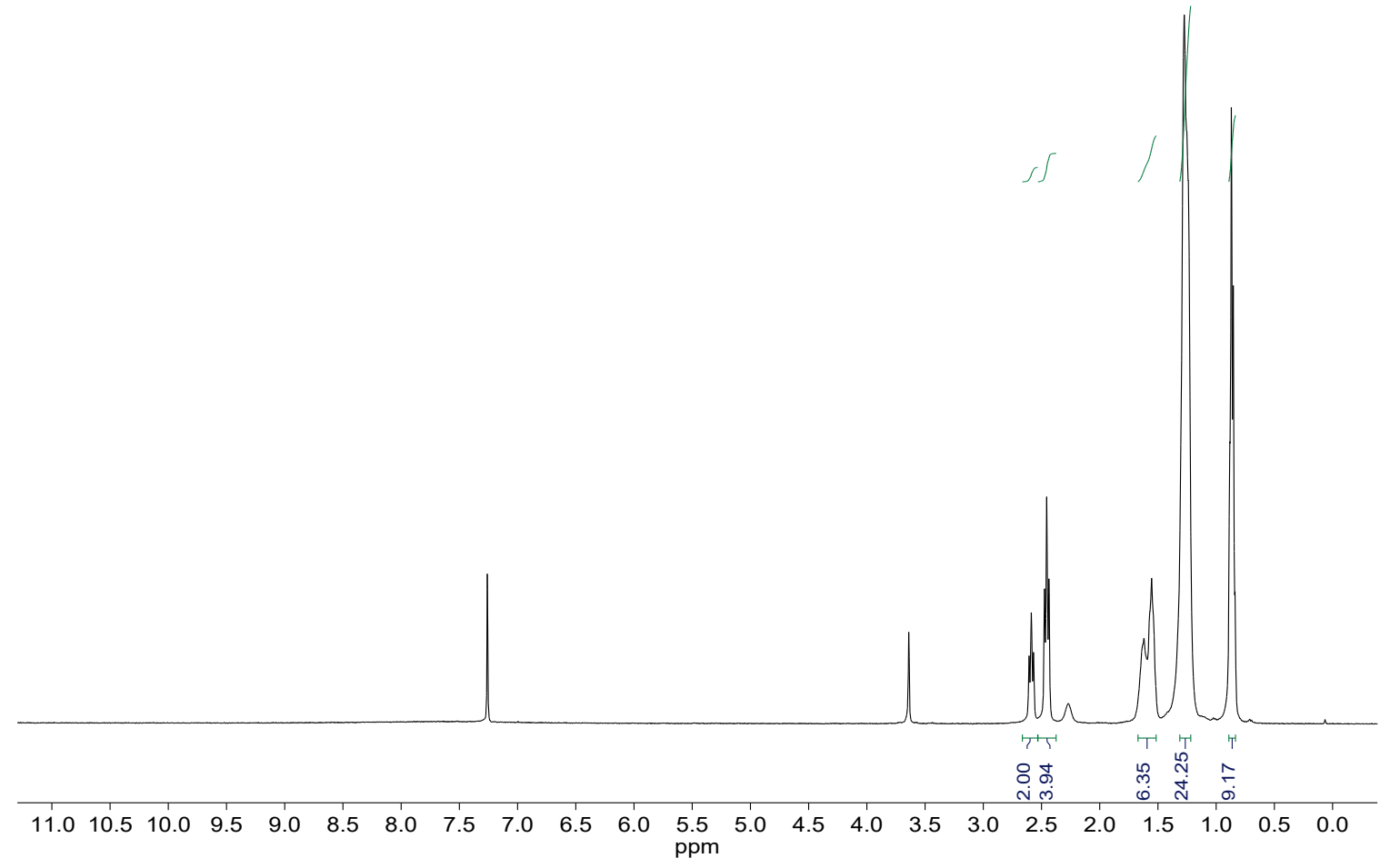

Magnification of the N-H signal:

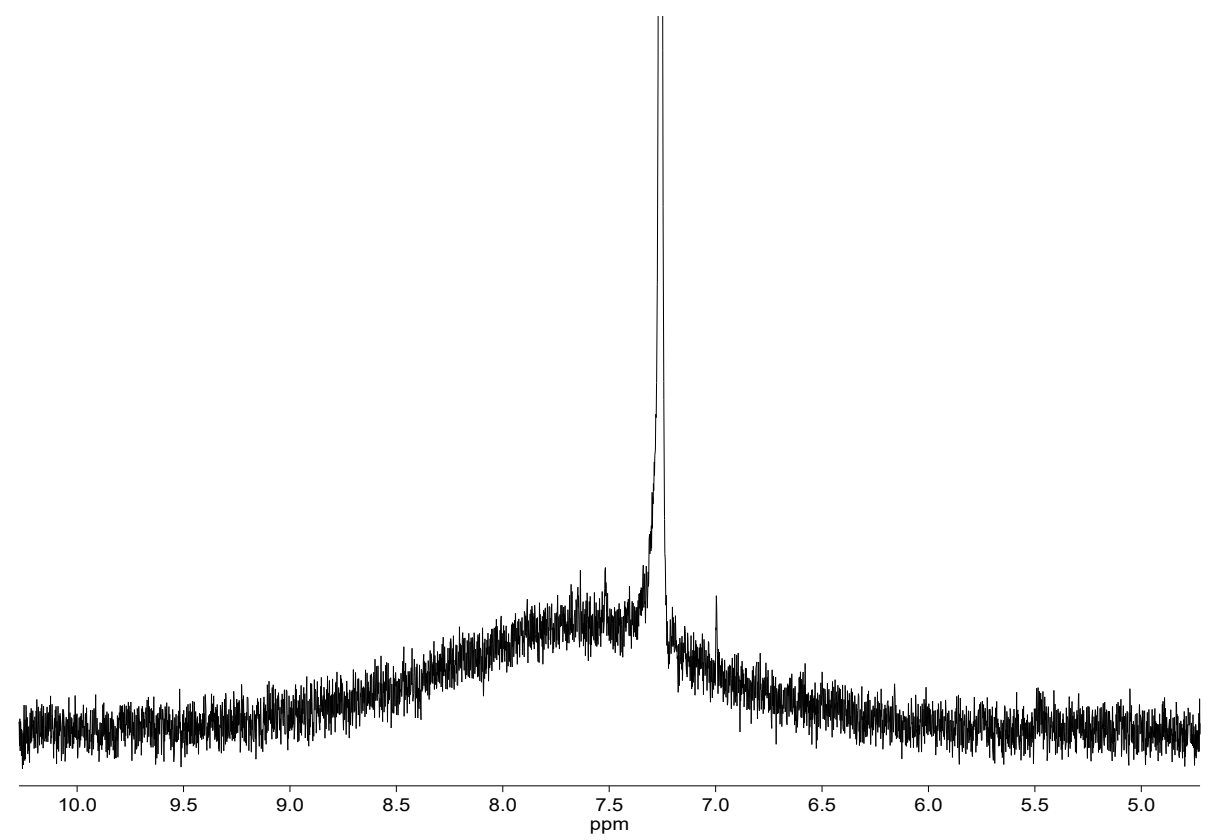


Compound 4-7 $\left.\left({ }^{13} \mathrm{C}_{\{}{ }^{1} \mathrm{H}\right\}, 101 \mathrm{MHz}, \mathrm{CDCl}_{3}\right)$

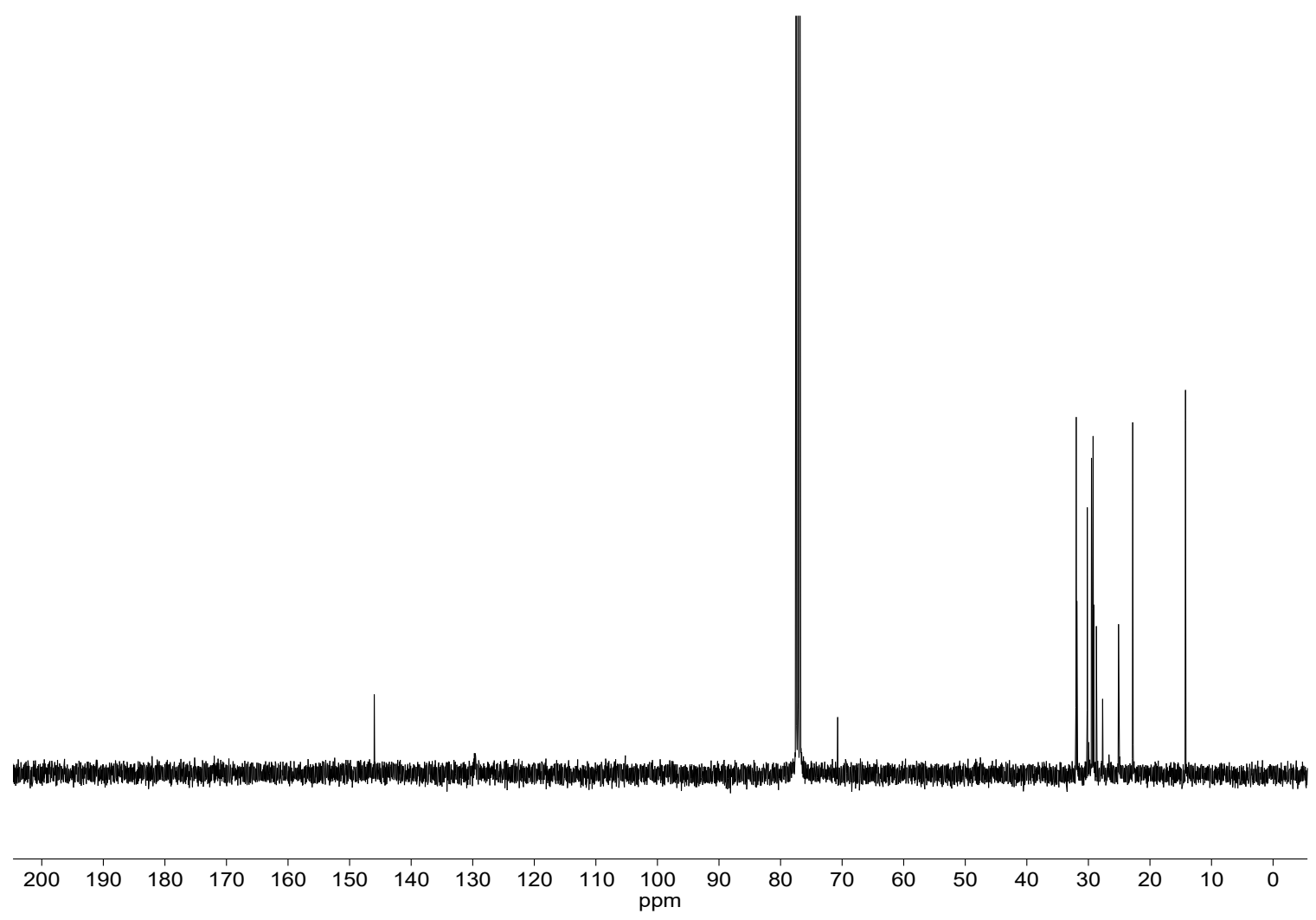


Compound 4-11 ( $\left.{ }^{1} \mathrm{H}, 300 \mathrm{MHz}, \mathrm{CDCl}_{3}\right)$

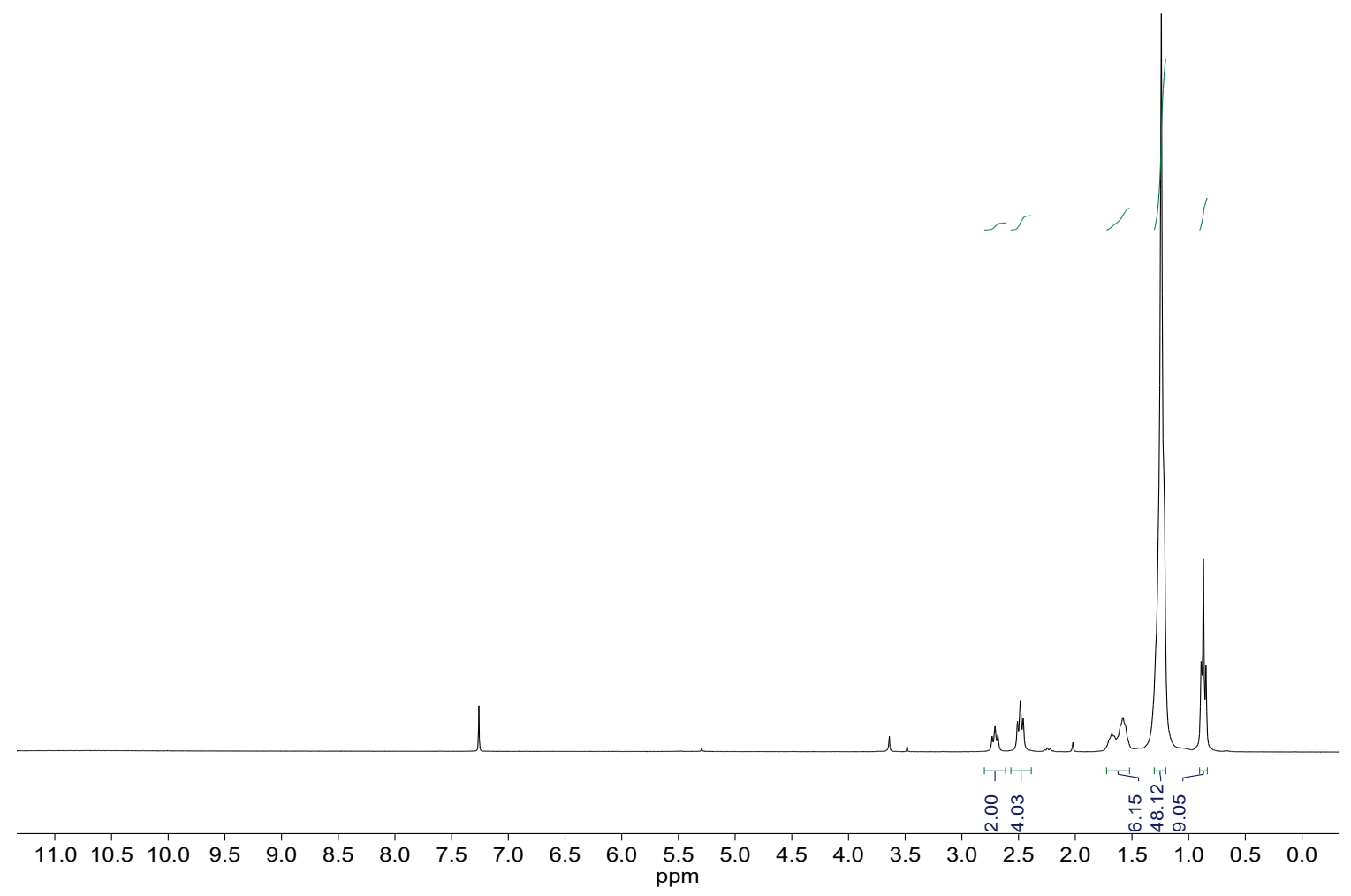

Magnification of the N-H signal:

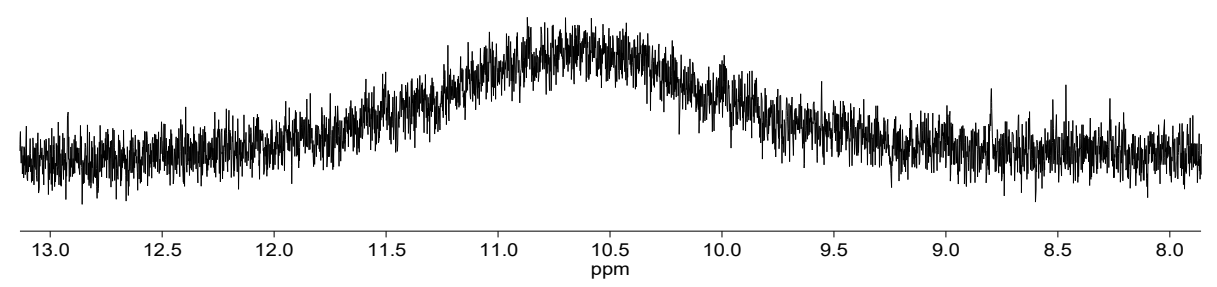


Compound 4-11 $\left.\left({ }^{13} \mathrm{C}_{\{}{ }^{1} \mathrm{H}\right\}, 75 \mathrm{MHz}, \mathrm{CDCl}_{3}\right)$

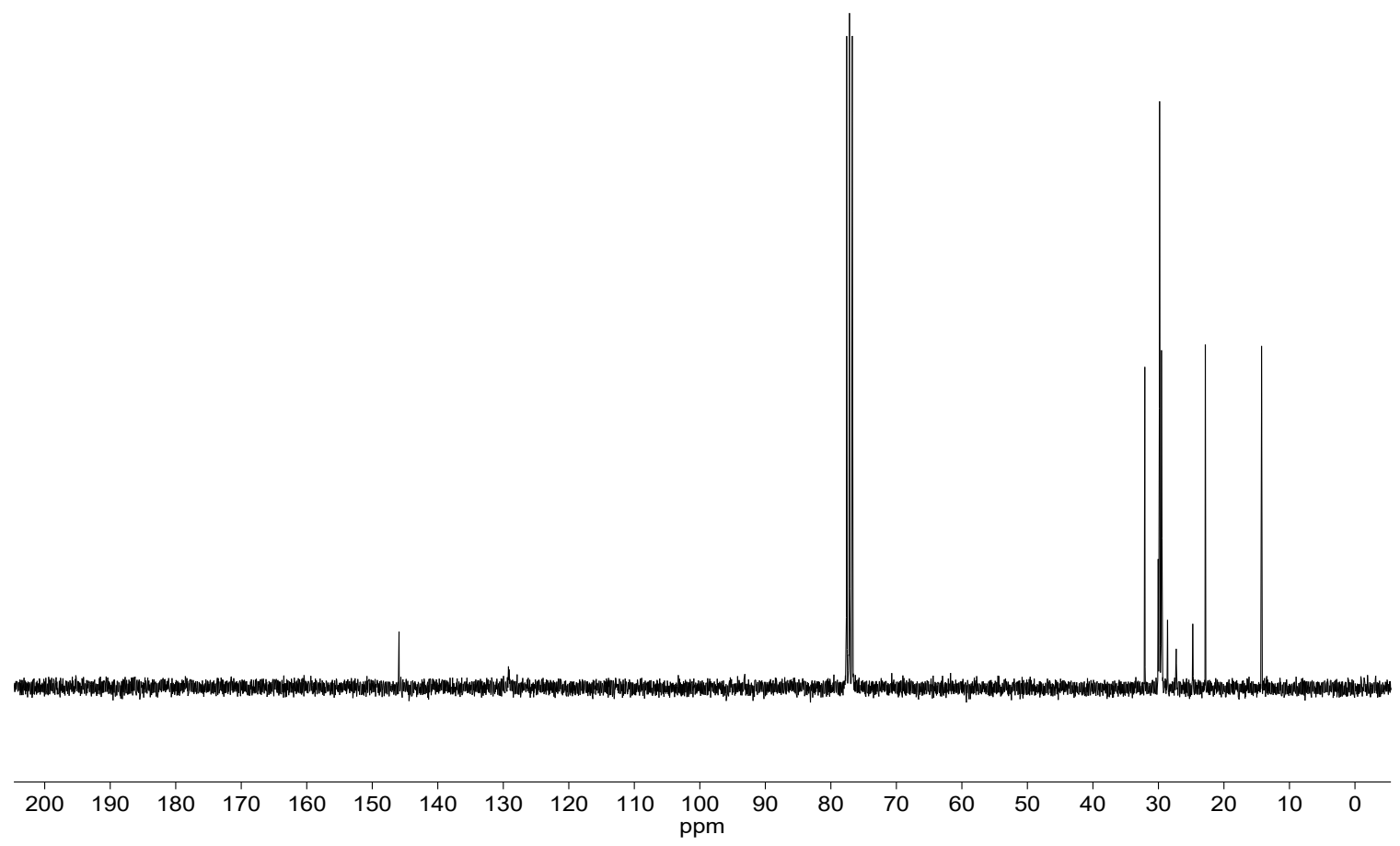


Compound 4-15 ( $\left.{ }^{1} \mathrm{H}, 300 \mathrm{MHz}, \mathrm{CDCl}_{3}\right)$

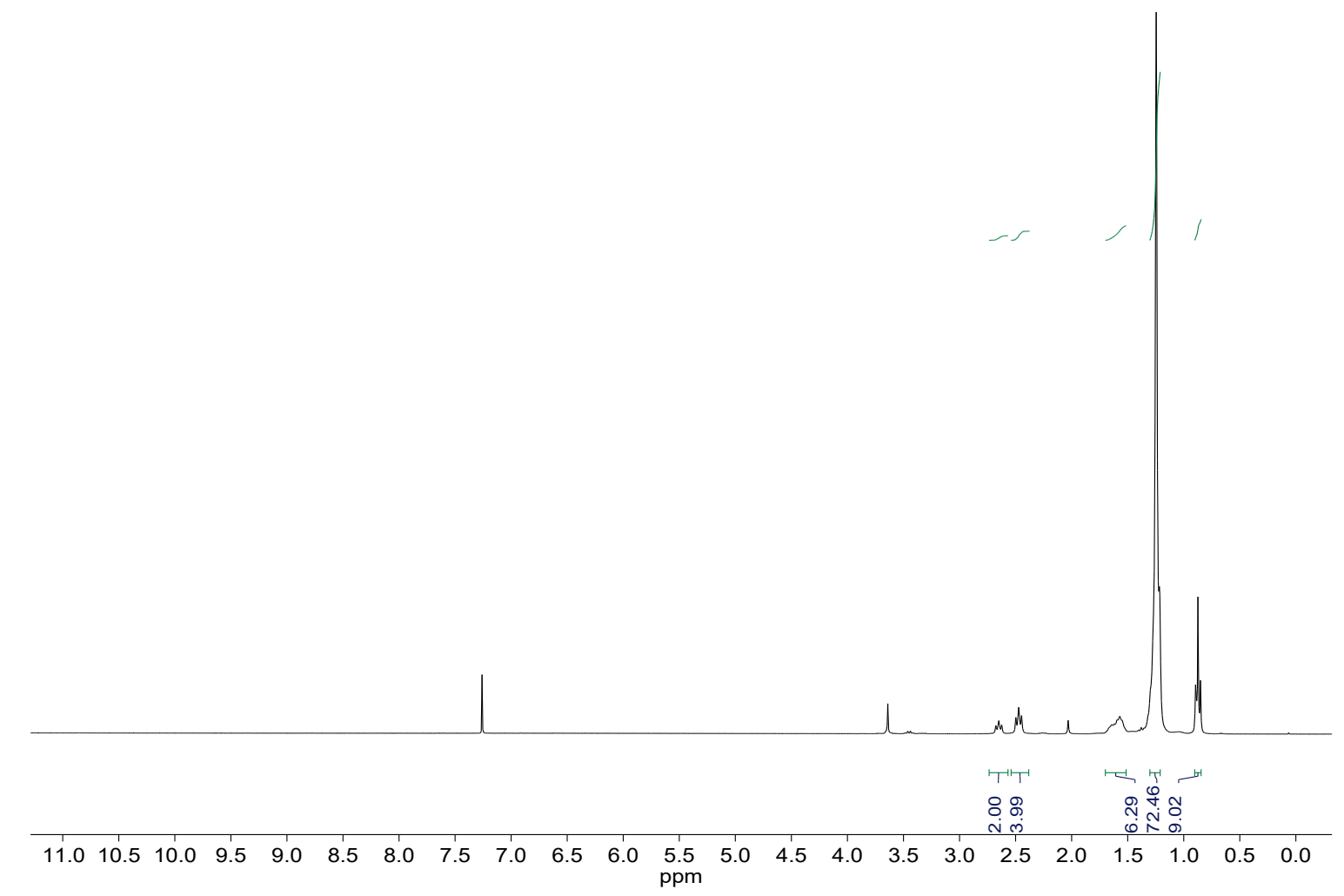

Magnification of the N-H signal:

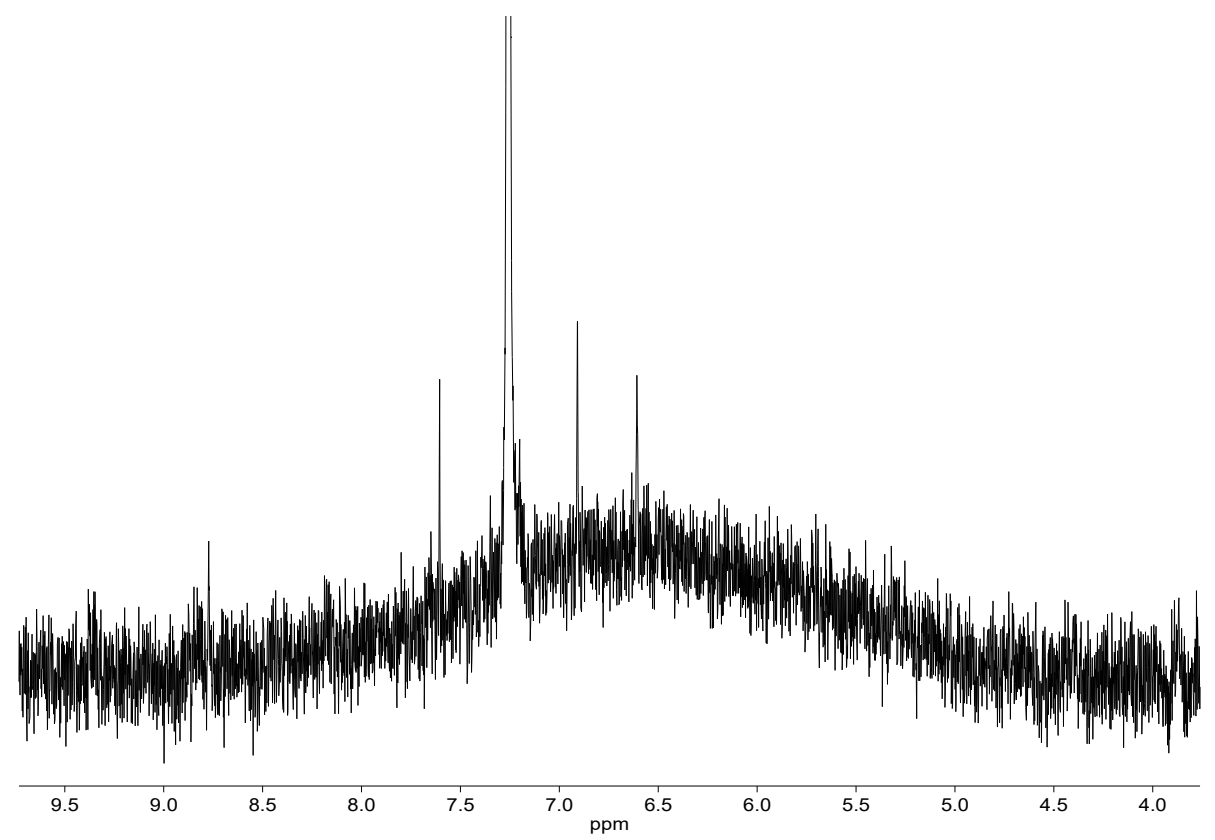


Compound 4-15 $\left.\left({ }^{13} \mathrm{C}_{\{}{ }^{1} \mathrm{H}\right\}, 75 \mathrm{MHz}, \mathrm{CDCl}_{3}\right)$

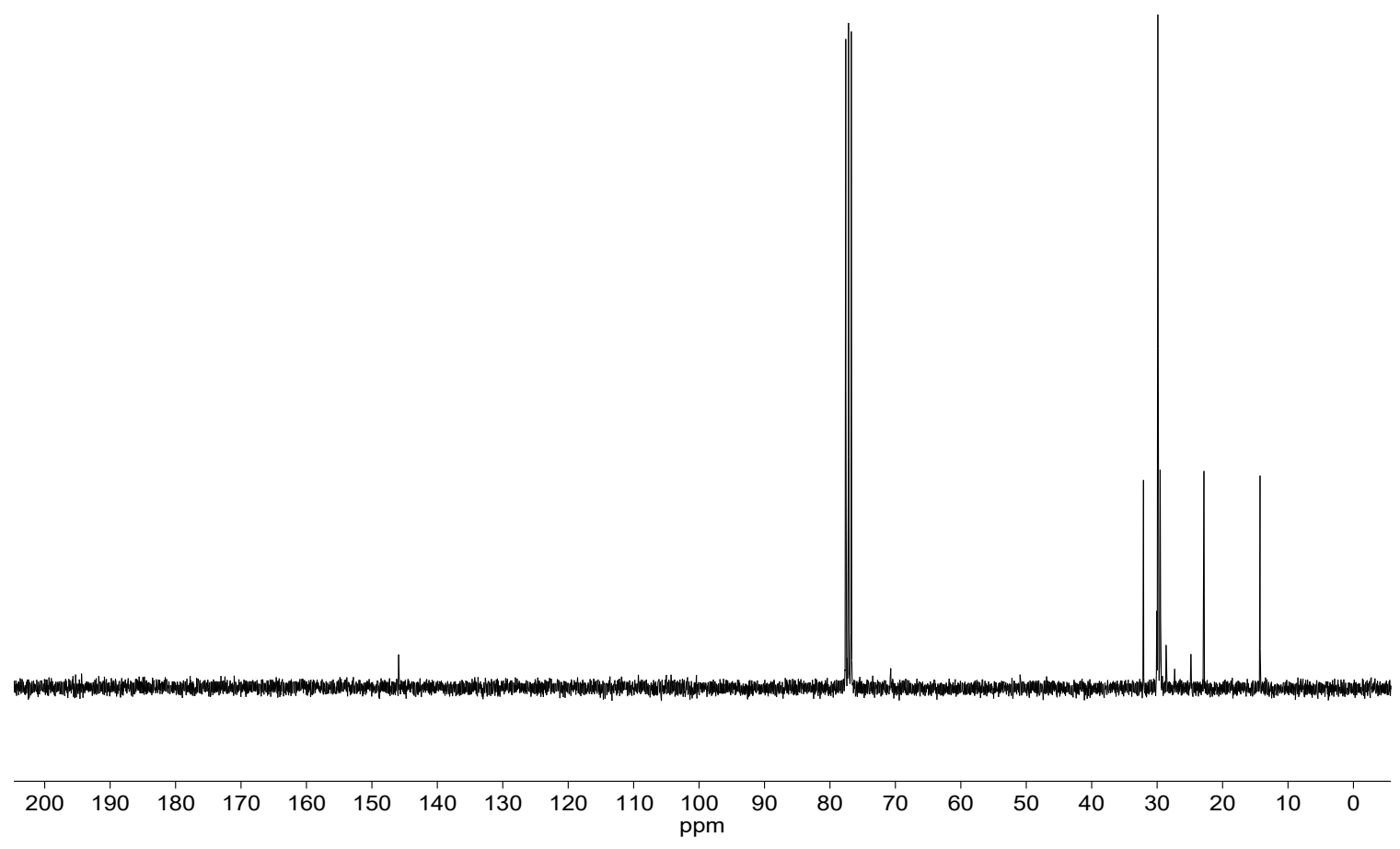


Compound 5-Br-7 ( $\left.{ }^{l} \mathrm{H}, 400 \mathrm{MHz}, \mathrm{CDCl}_{3}\right)$

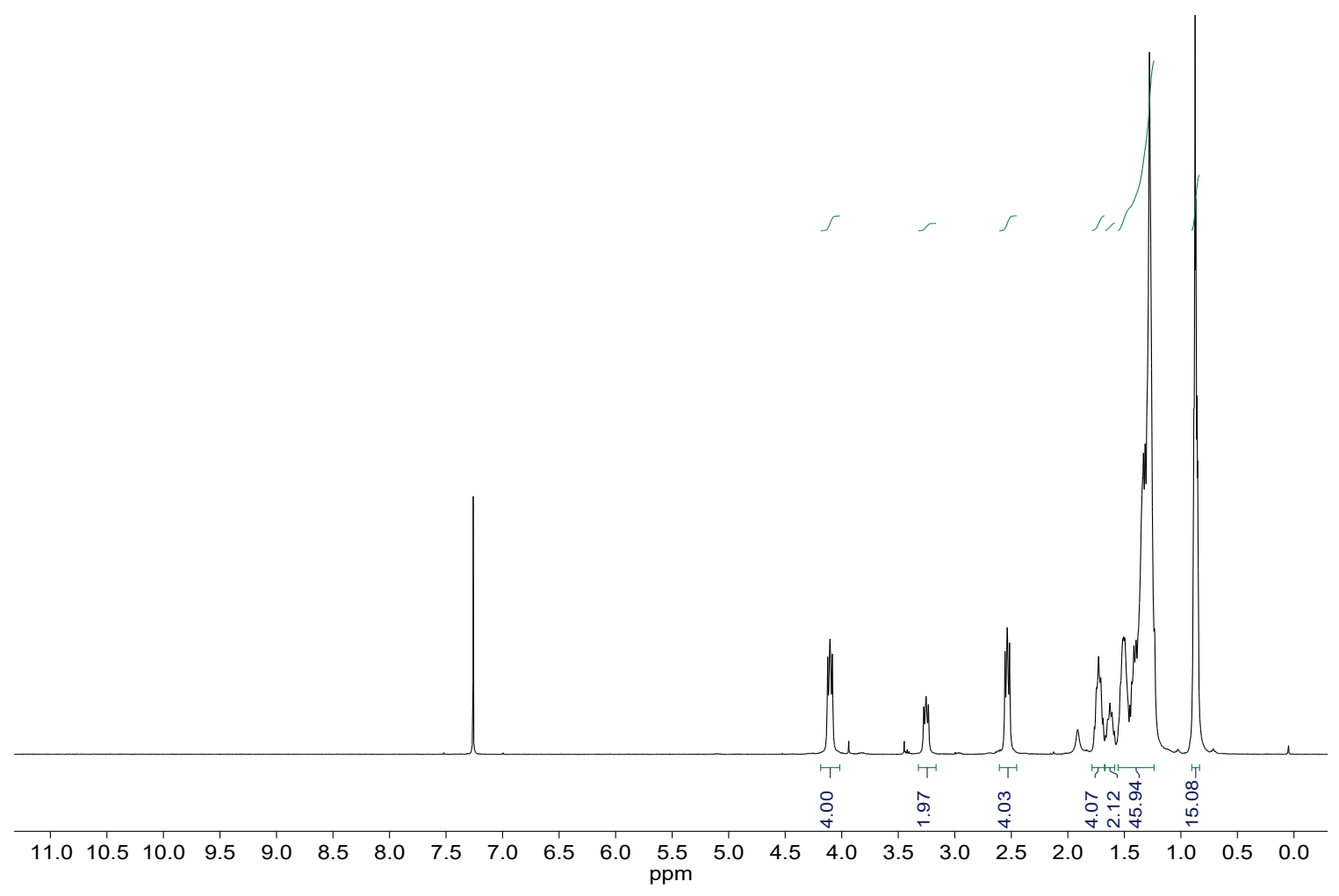

Compound 5-Br-7 $\left({ }^{13} \mathrm{C}_{\{}^{\left\{^{1} \mathrm{H}\right\}, 101 \mathrm{MHz}, \mathrm{CDCl}} 3\right)$

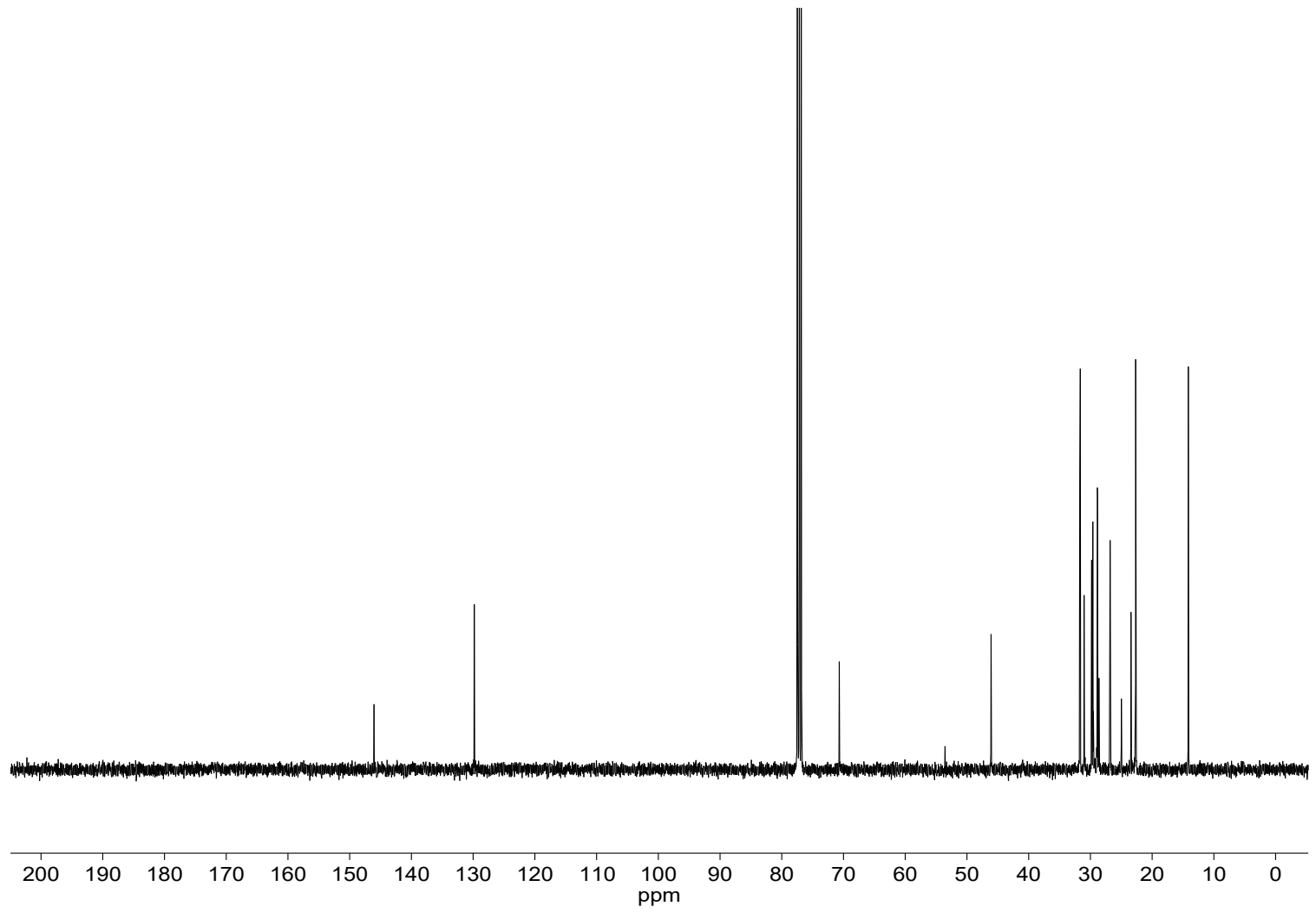

S27 
Compound 5-Br-11 ( $\left.{ }^{1} \mathrm{H}, 300 \mathrm{MHz}, \mathrm{CDCl}_{3}\right)$

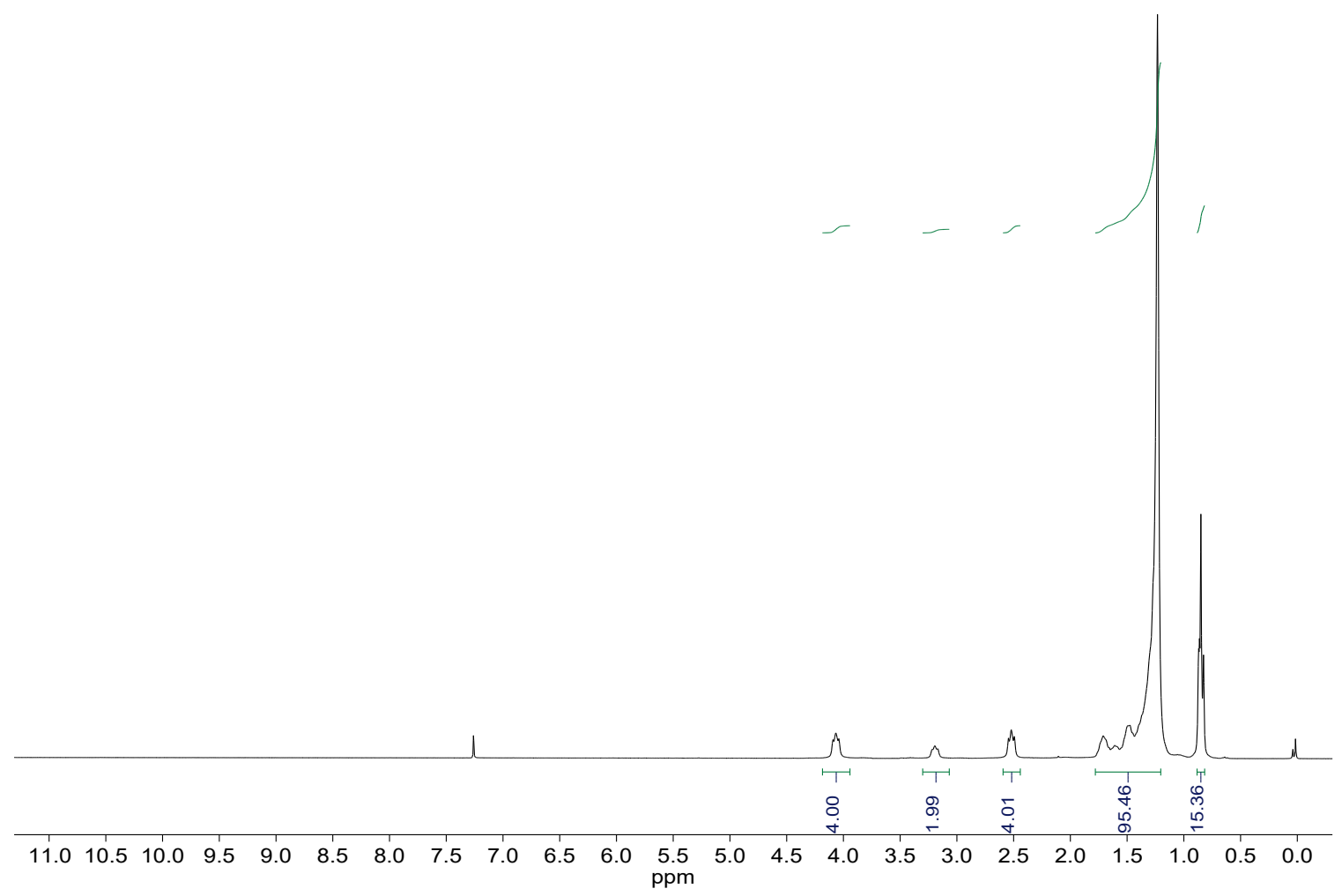

Compound 5-Br-11 $\left({ }^{13} \mathrm{C}_{\{}^{1} \mathrm{H}_{\}}, 75 \mathrm{MHz}, \mathrm{CDCl}_{3}\right)$

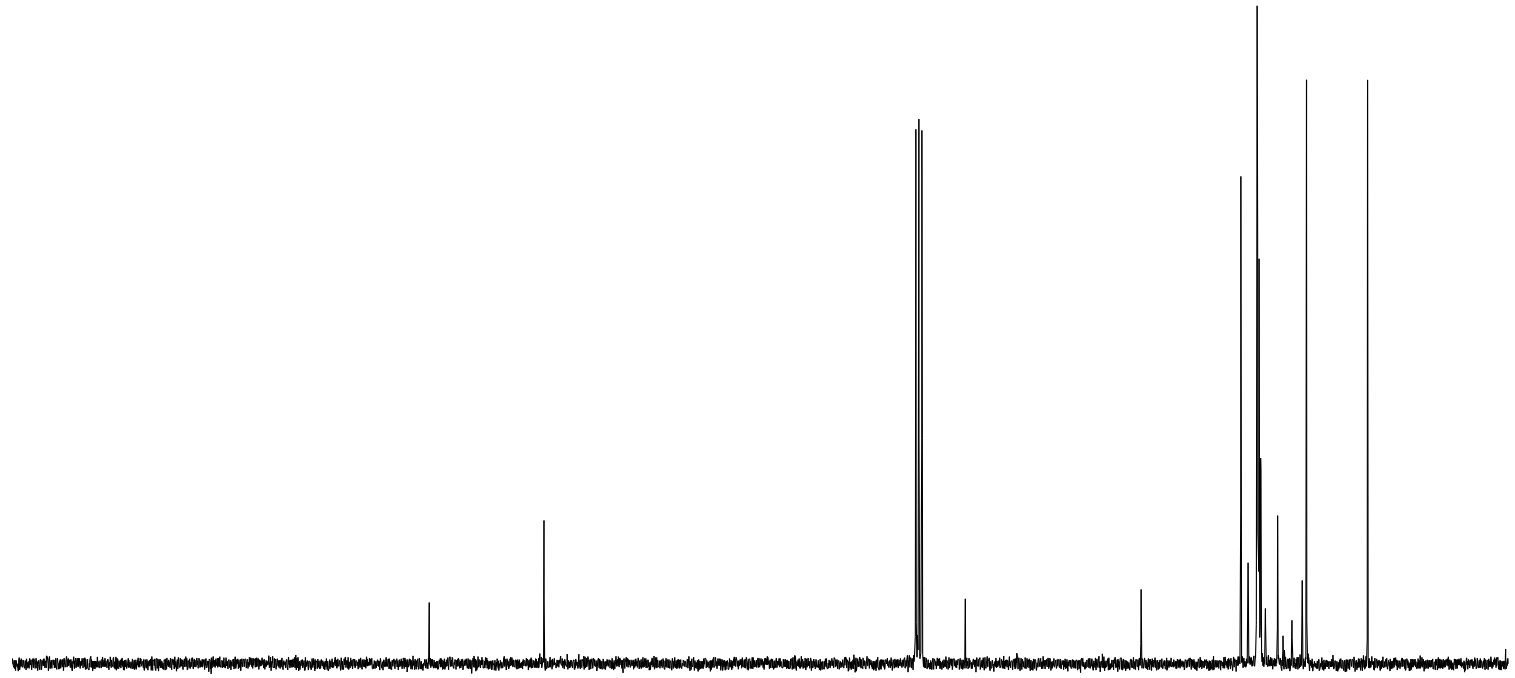

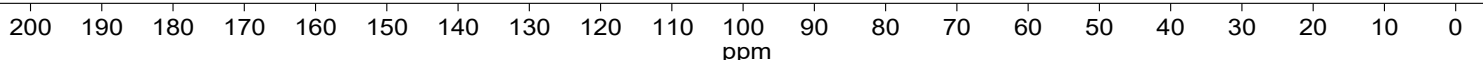


Compound 5-Br-15 ( $\left.{ }^{l} \mathrm{H}, 400 \mathrm{MHz}, \mathrm{CDCl}_{3}\right)$

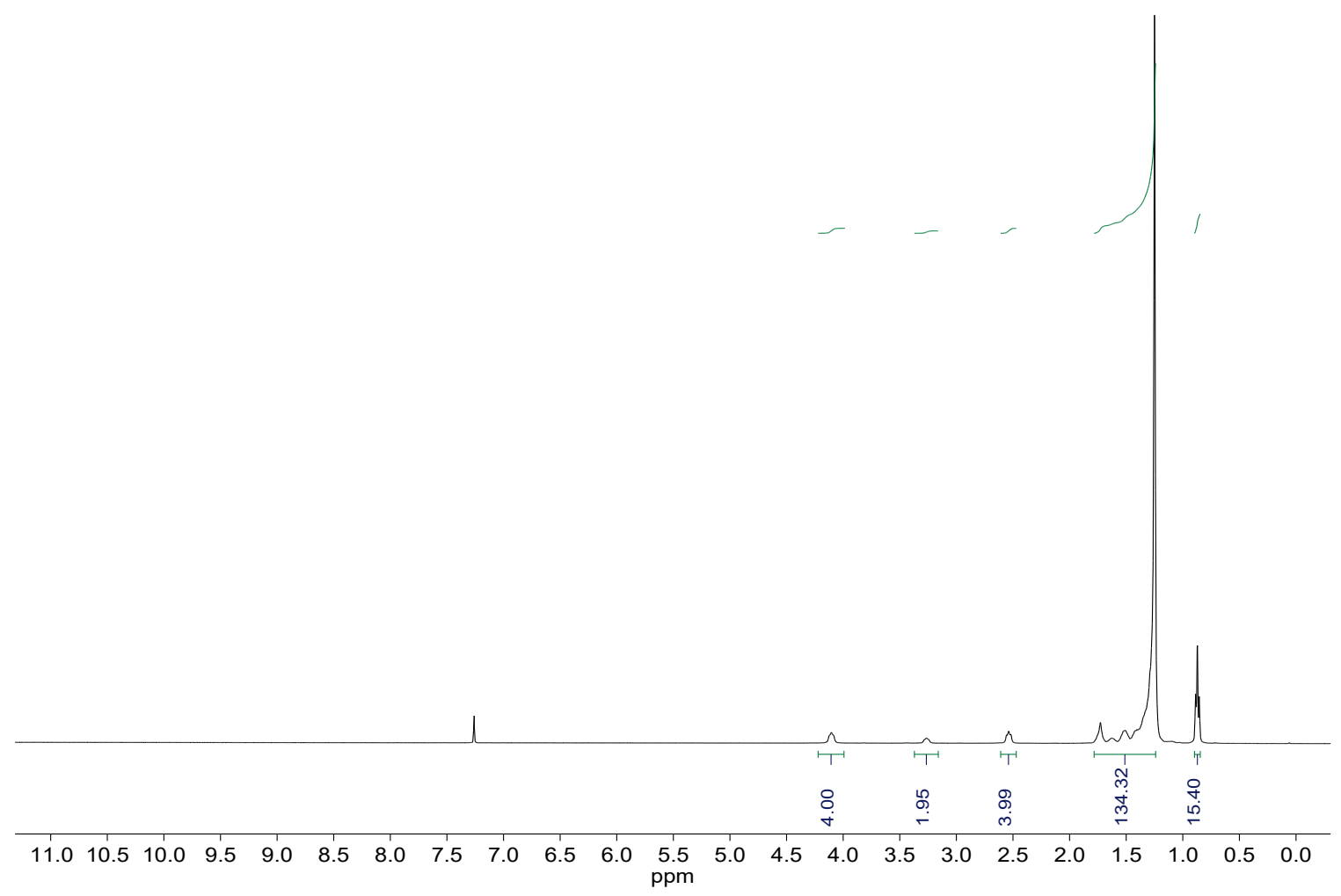

Compound 5-Br-15 $\left({ }^{13} \mathrm{C}_{\{}^{1} \mathrm{H}_{\}}, 101 \mathrm{MHz}, \mathrm{CDCl}_{3}\right)$

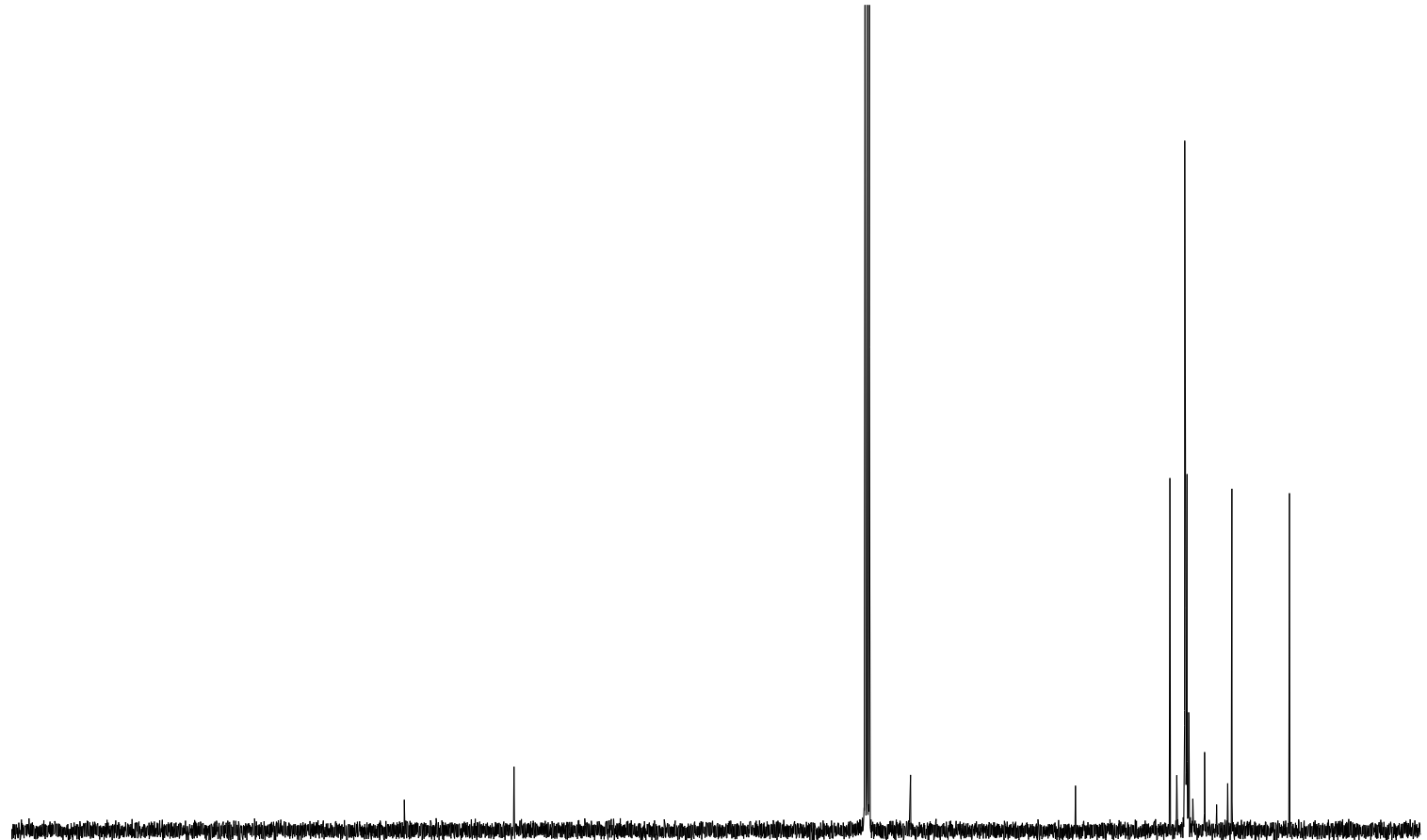

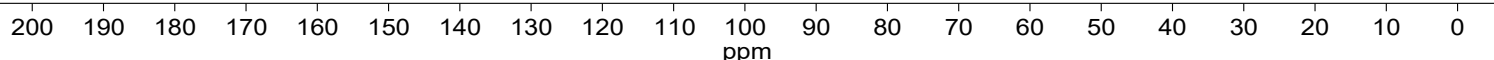


Compound 5-NO $\mathrm{O}_{3}-1\left({ }^{1} \mathrm{H}, 400 \mathrm{MHz}, \mathrm{DMSO}-d_{6}\right)$

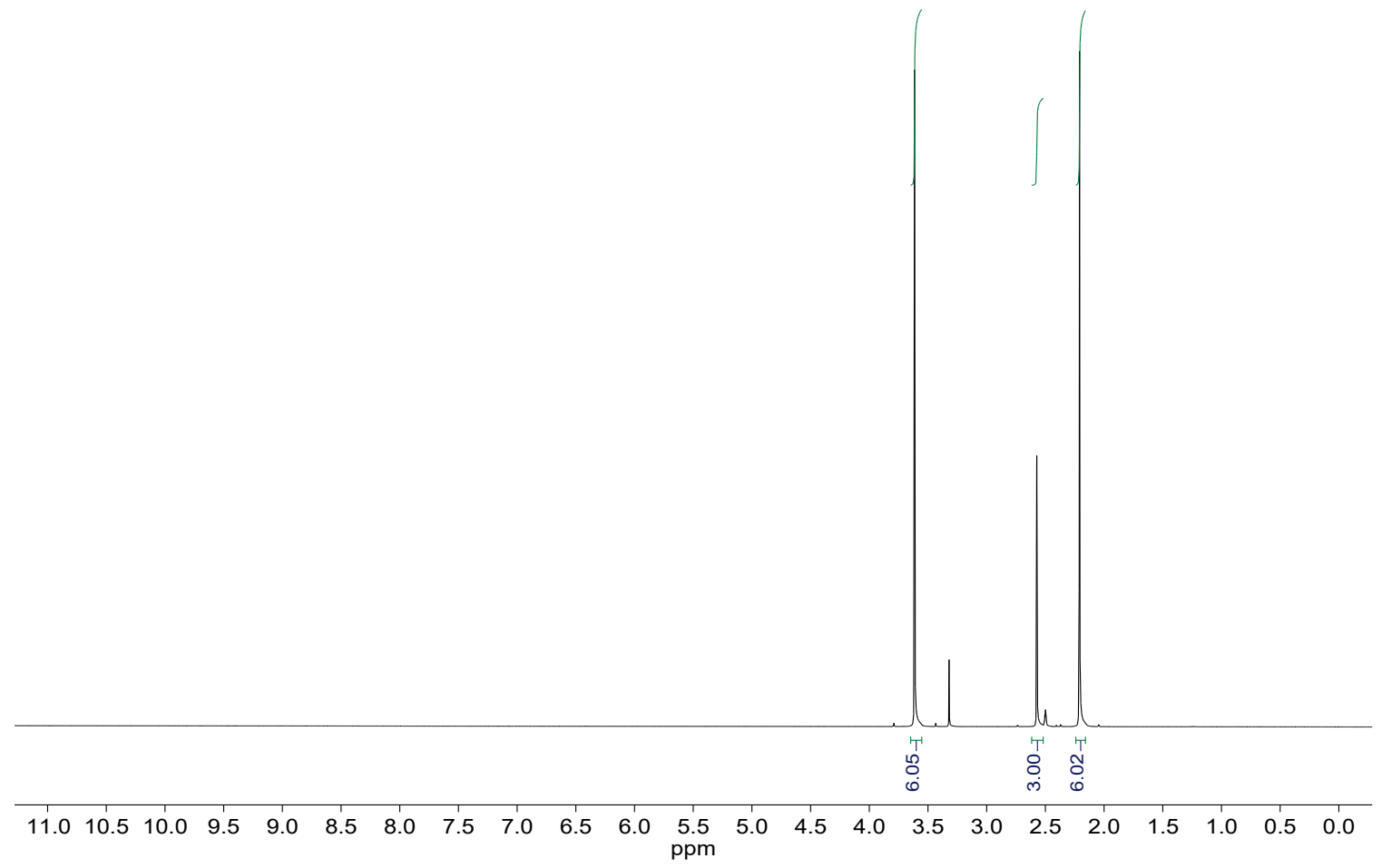

Compound 5-NO $\left.{ }_{3}-1{ }^{13} C_{\{}^{\prime 1} H\right\}, 101 \mathrm{MHz}$, DMSO-d $\left.d_{6}\right)$

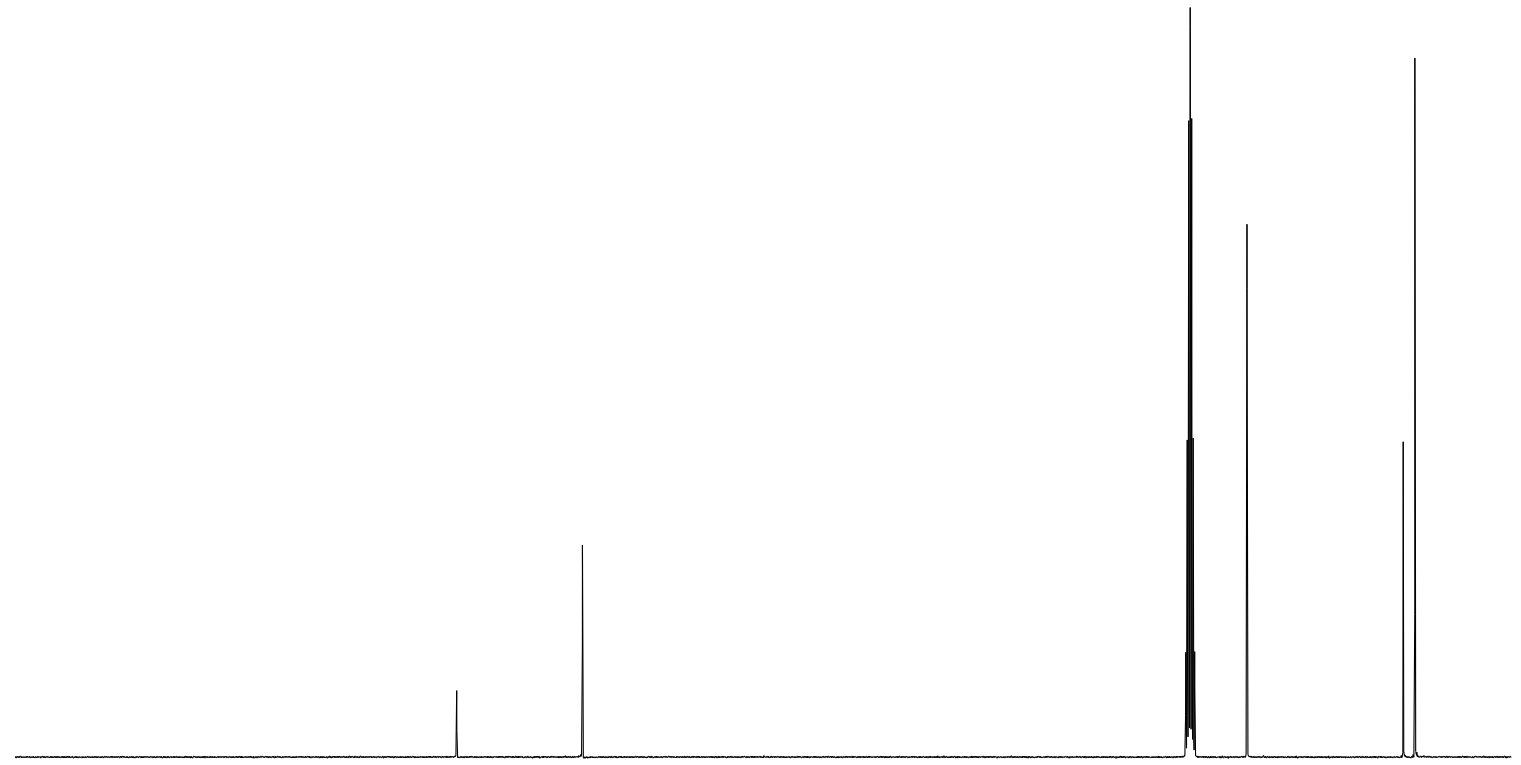

$\begin{array}{lllllllllllllllllllll}200 & 190 & 180 & 170 & 160 & 150 & 140 & 130 & 120 & 110 & \begin{array}{c}100 \\ \mathrm{ppm}\end{array} & 90 & 80 & 70 & 60 & 50 & 40 & 30 & 20 & 10 & 0\end{array}$ 
Compound 5-NO $\mathbf{3}^{-7}$ ( $\left.{ }^{1} \mathrm{H}, 400 \mathrm{MHz}, \mathrm{CDCl}_{3}\right)$

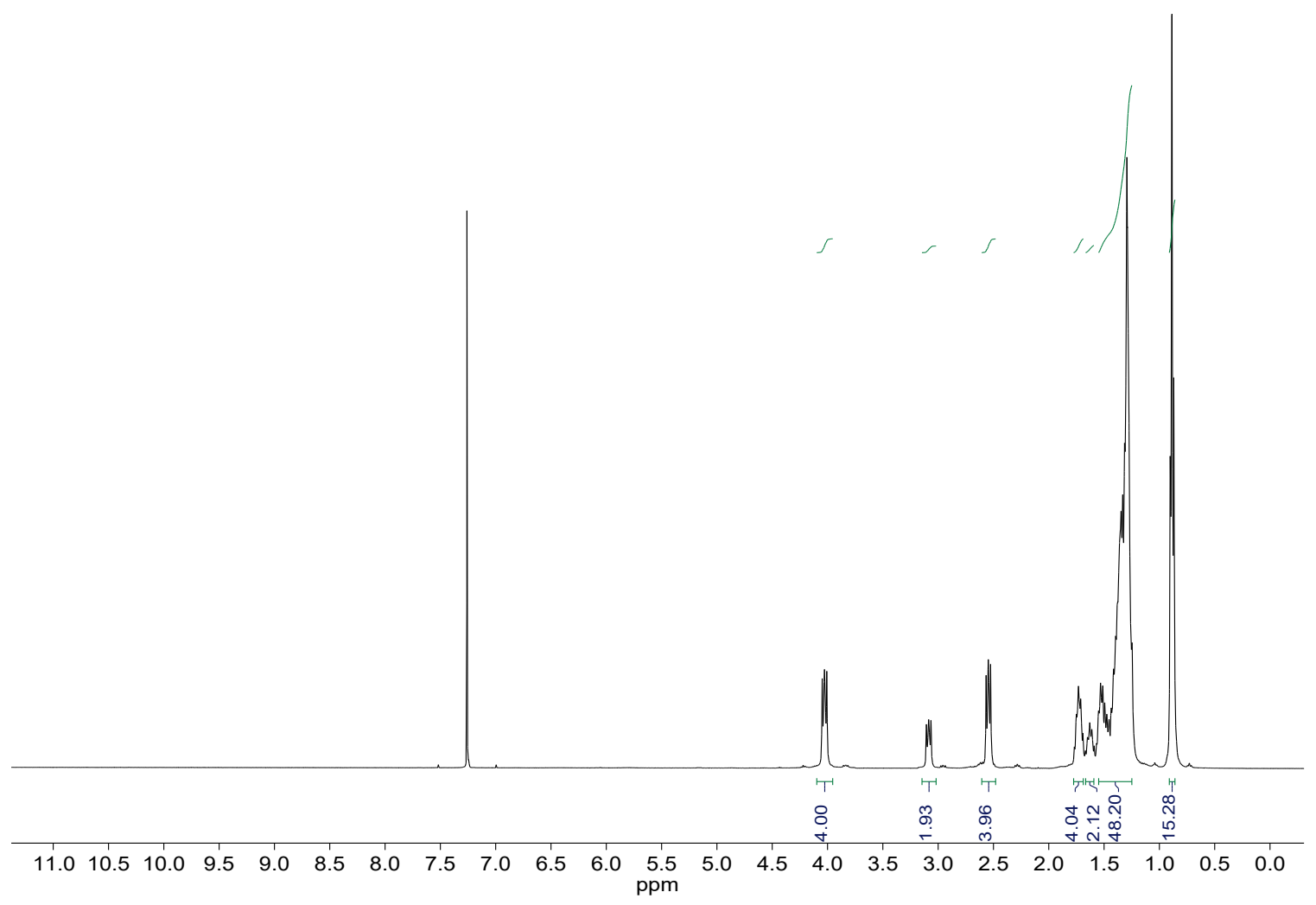

Compound 5-NO ${ }_{3-7}-7\left({ }^{13} \mathrm{C}_{\{}^{1} \mathrm{H}_{\}}, 101 \mathrm{MHz}, \mathrm{CDCl}_{3}\right)$

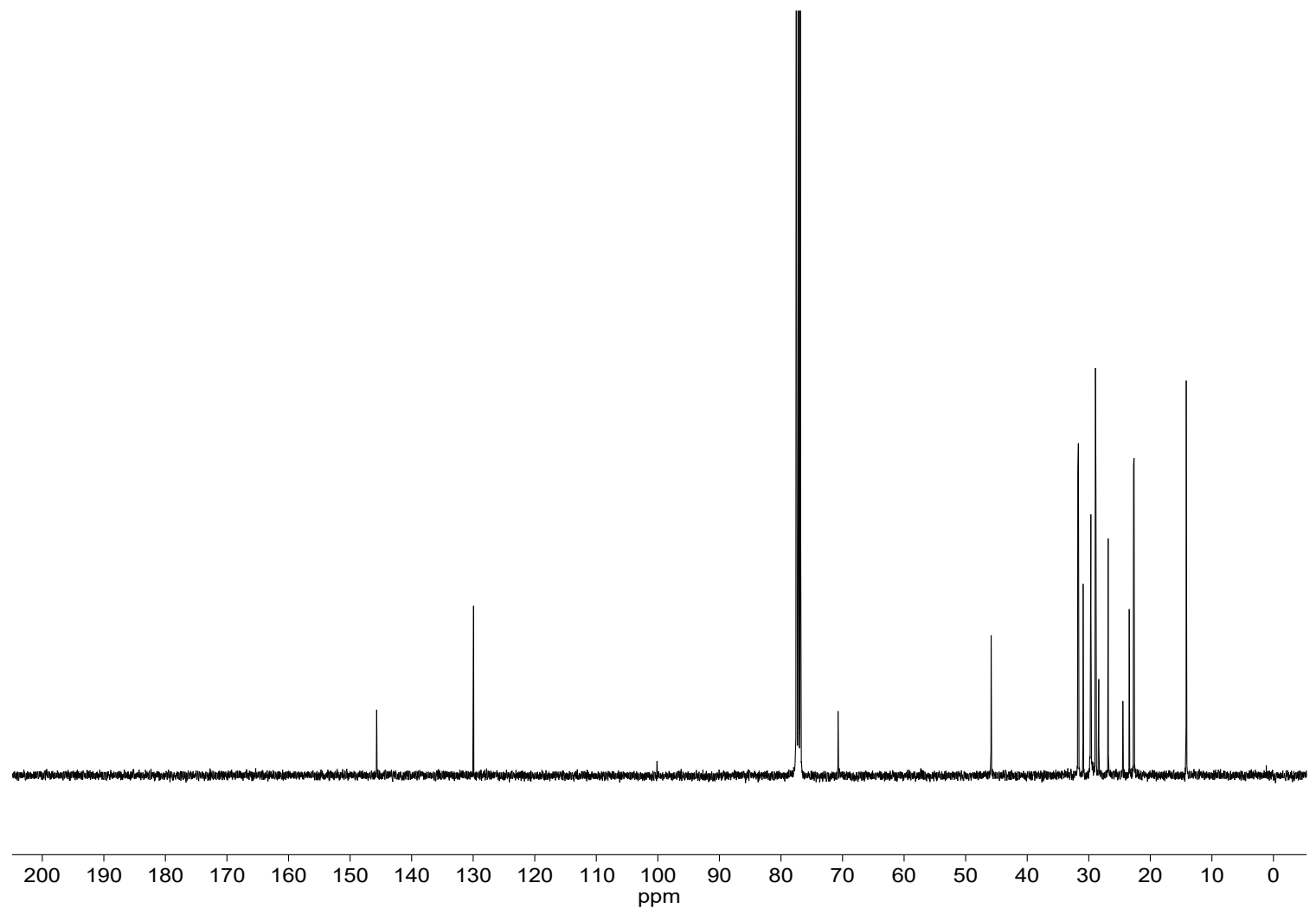

S31 
Compound 5-NO $\mathbf{O}_{3}-11\left({ }^{1} \mathrm{H}, 400 \mathrm{MHz}, \mathrm{CDCl}_{3}\right)$

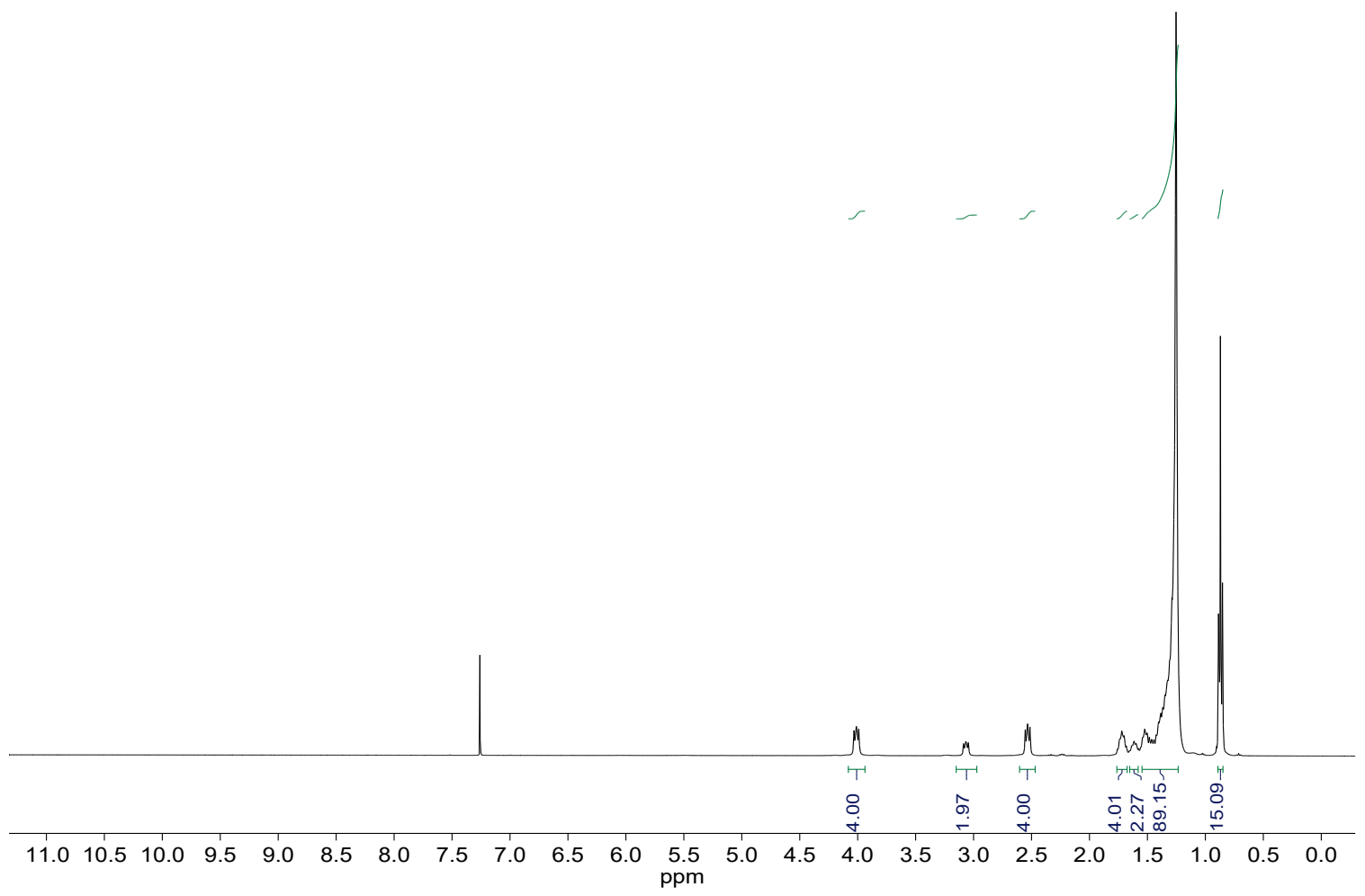

Compound 5-NO $\left.\mathrm{O}_{3}-11\left({ }^{13} \mathrm{C}_{\{}{ }^{1} \mathrm{H}\right\}, 101 \mathrm{MHz}, \mathrm{CDCl}_{3}\right)$

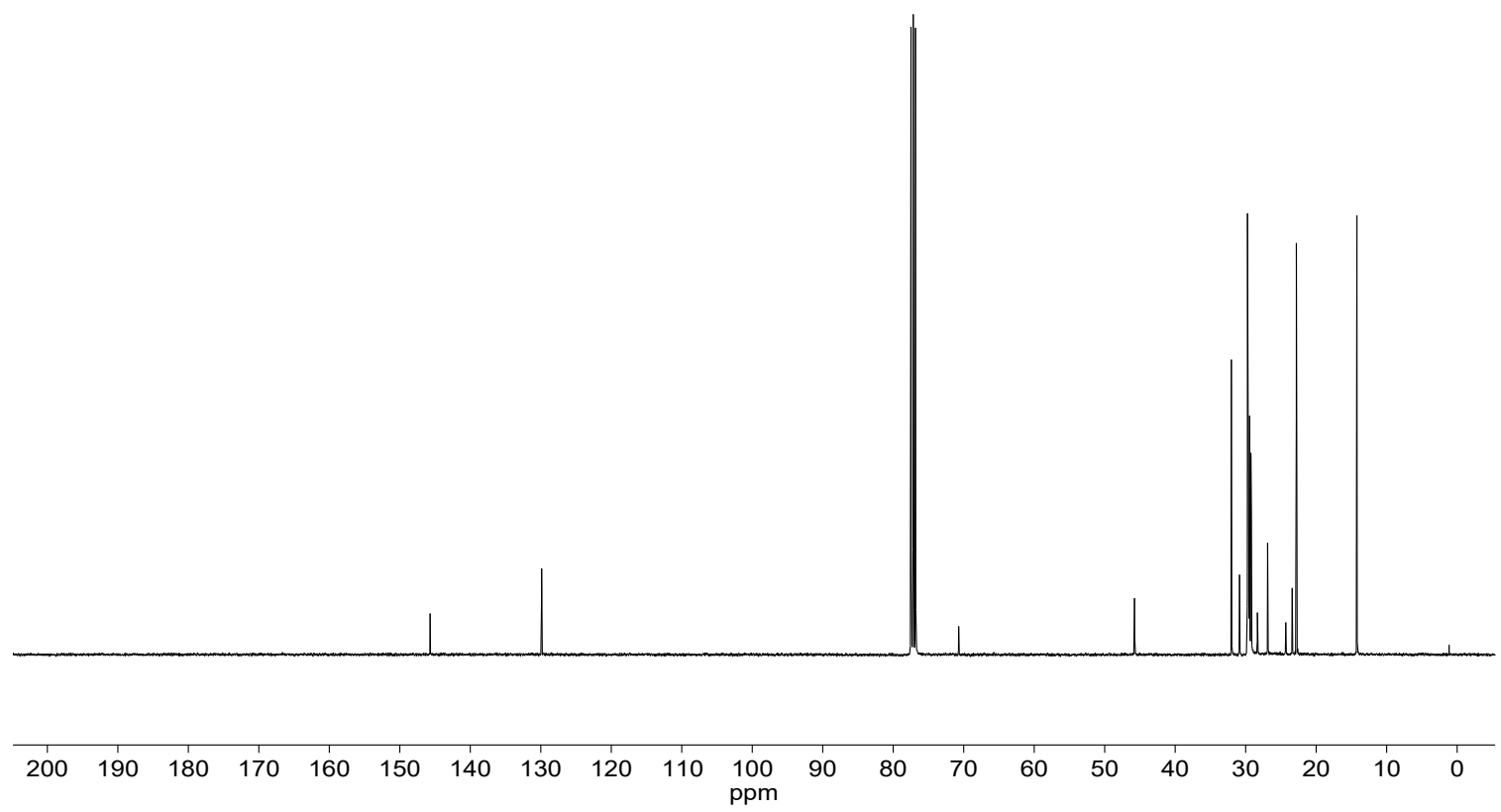

S32 
Compound 5-NO $-15\left({ }^{1} \mathrm{H}, 400 \mathrm{MHz}, \mathrm{CDCl}_{3}\right)$

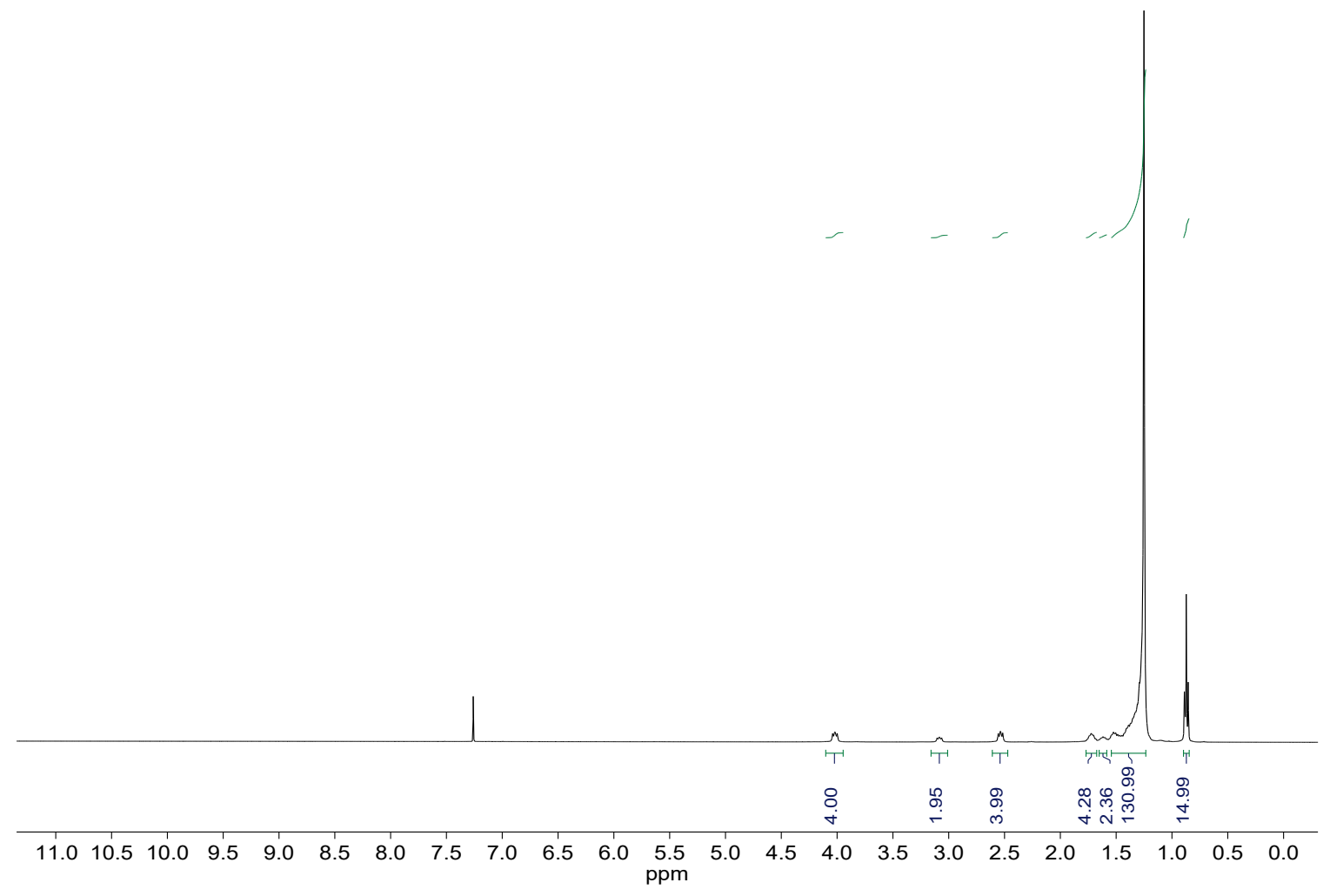

Compound 5-NO $\left.\mathrm{O}_{3}-15\left({ }^{13} \mathrm{C}_{\{}^{\prime l} \mathrm{H}\right\}, 101 \mathrm{MHz}, \mathrm{CDCl}_{3}\right)$

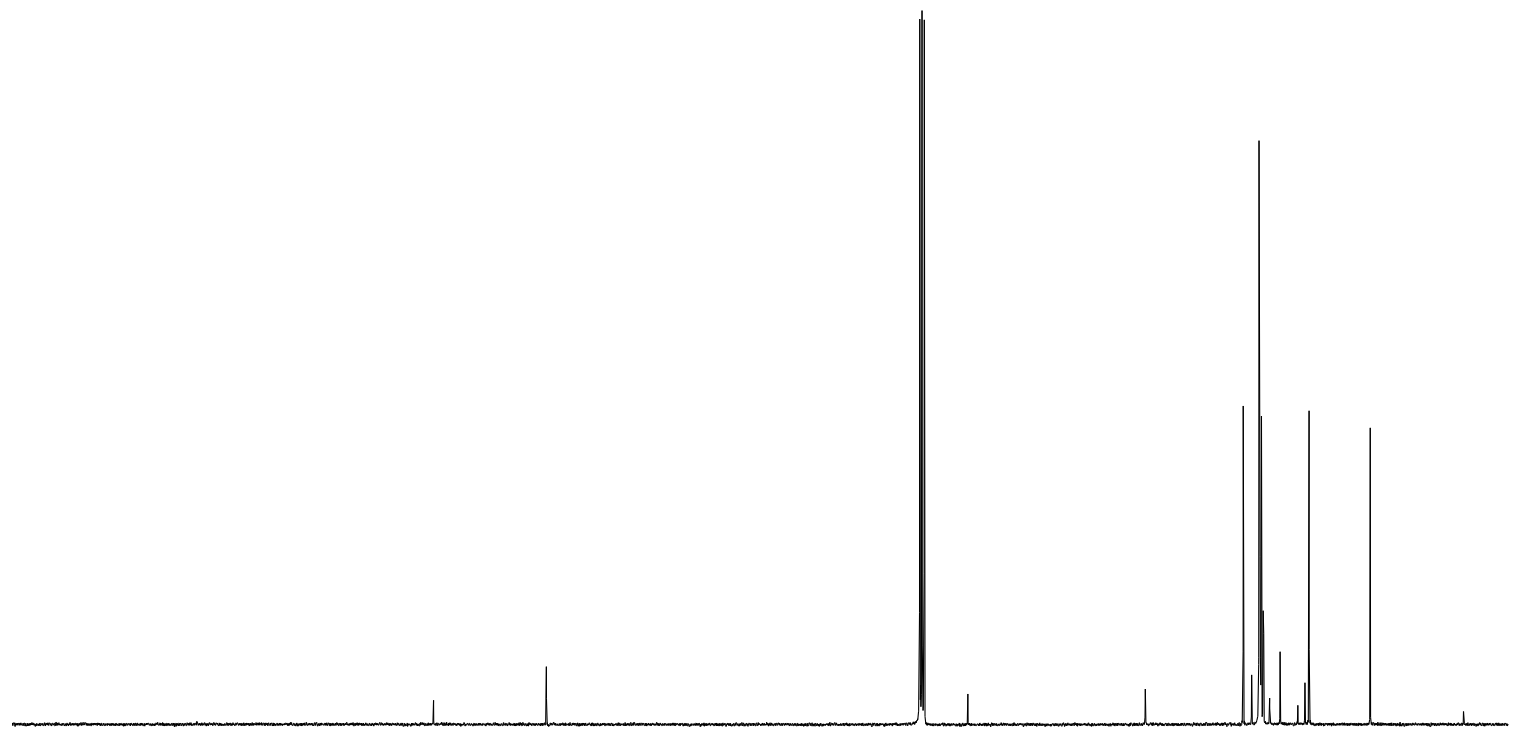

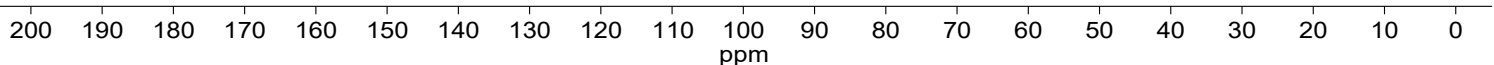


Compound 5-Cp(CN) ${ }_{5}-1\left({ }^{1} H, 400 \mathrm{MHz}, \mathrm{DMSO}-d_{6}\right)$

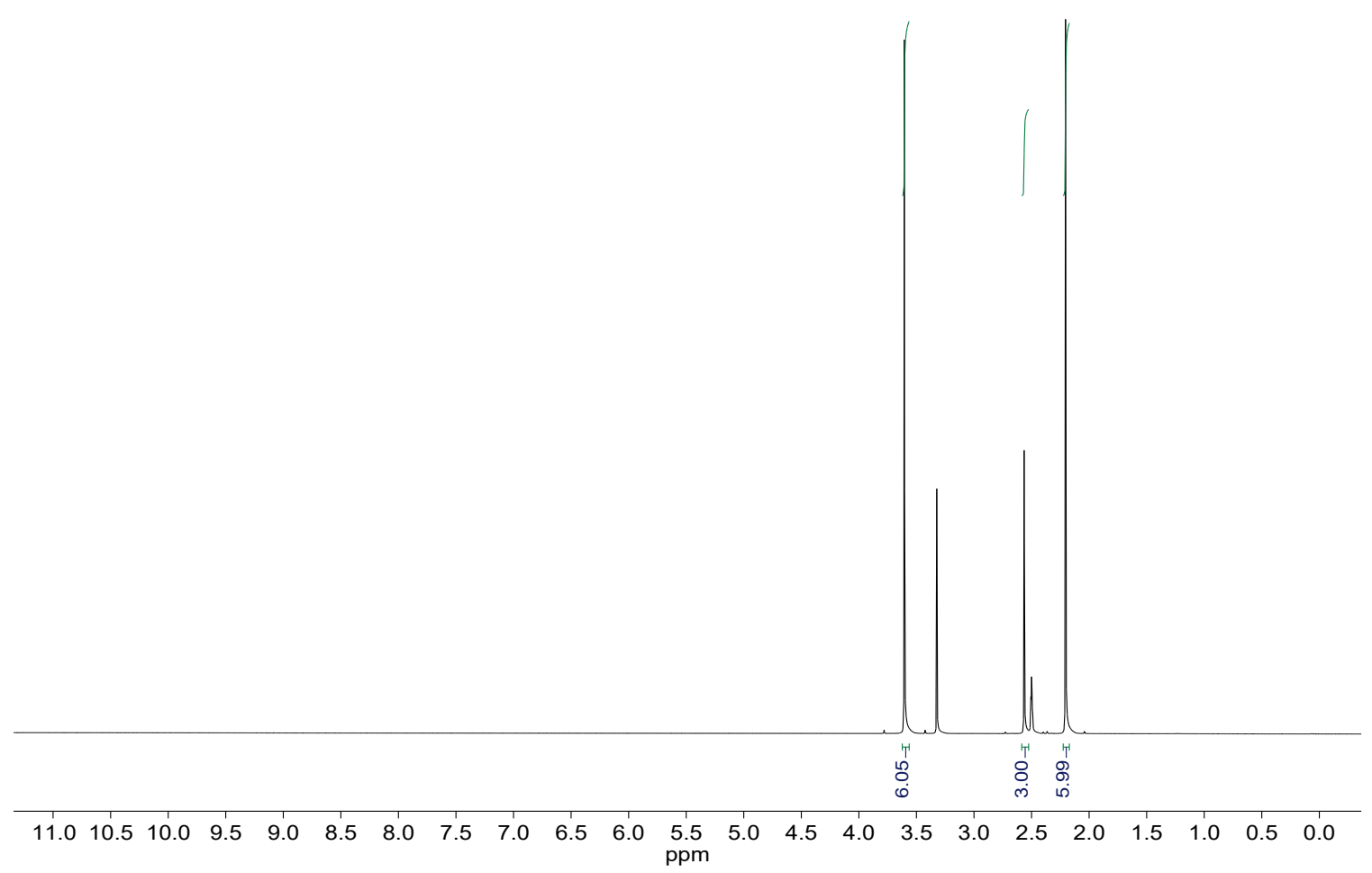

Compound 5-Cp(CN) ${ }_{5-1}\left({ }^{13} C_{\{}{ }^{1} H\right\}, 101 \mathrm{MHz}$, DMSO-d $\left.d_{6}\right)$

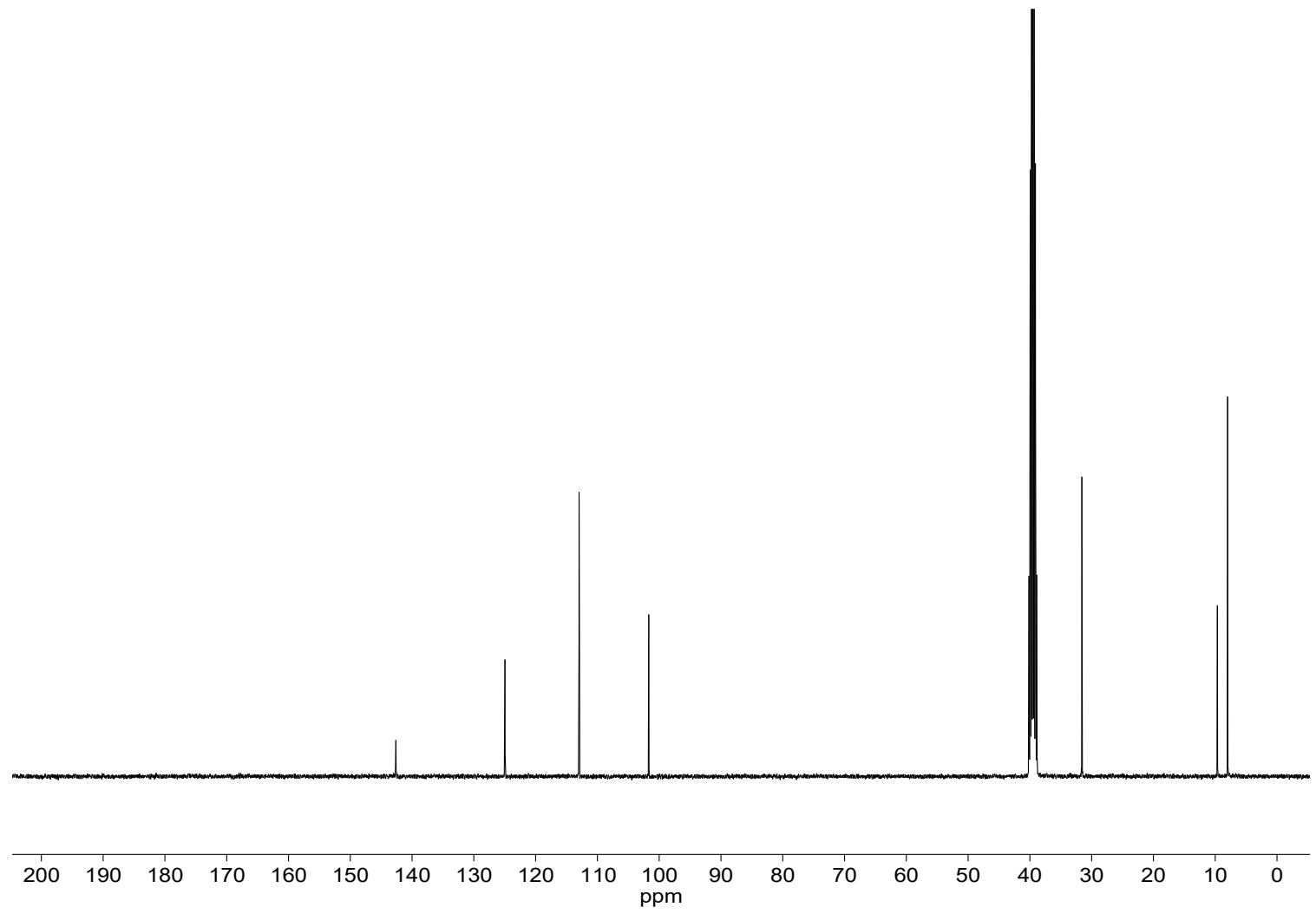

S34 
Compound 5-Cp(CN) $5^{-7}\left({ }^{l} \mathrm{H}, 400 \mathrm{MHz}, \mathrm{CDCl}_{3}\right)$

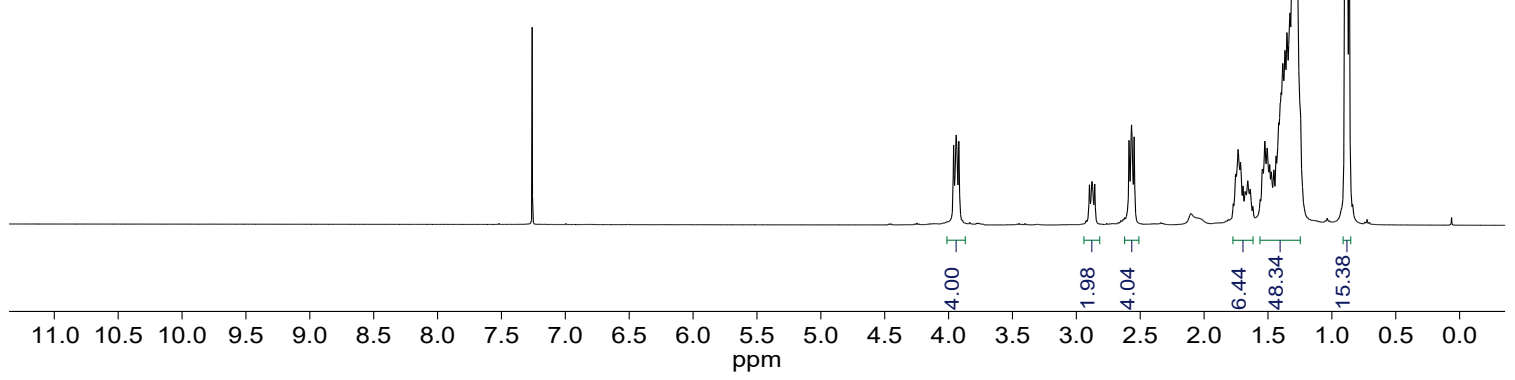

Compound 5-Cp(CN) $\left.{ }_{5^{-7}}\left(^{13} C_{\{}^{\{l} H\right\}, 101 \mathrm{MHz}, \mathrm{CDCl}_{3}\right)$

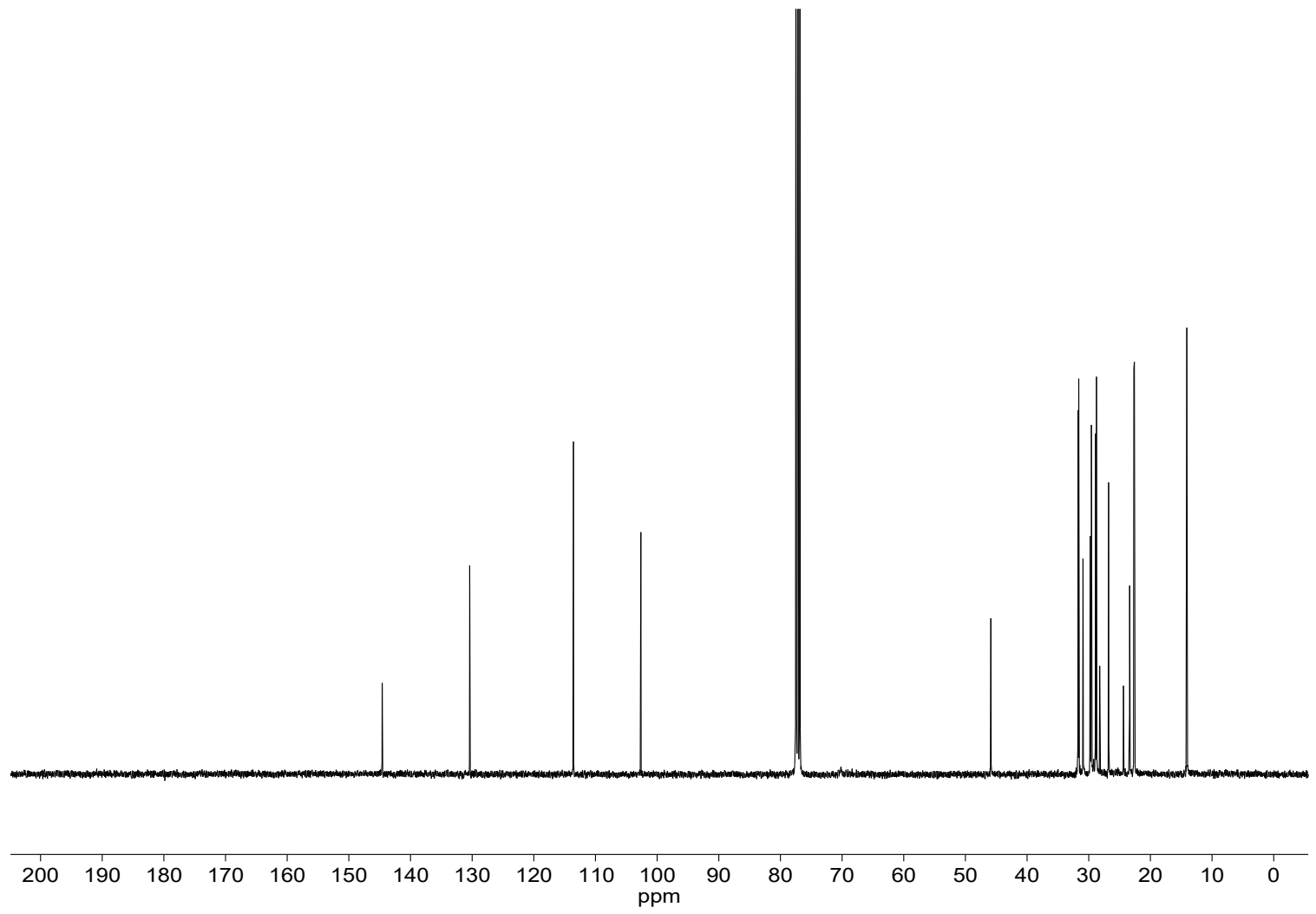

S35 
Compound 5-Cp(CN) $)^{-11}\left({ }^{1} \mathrm{H}, 400 \mathrm{MHz}, \mathrm{CDCl}_{3}\right)$

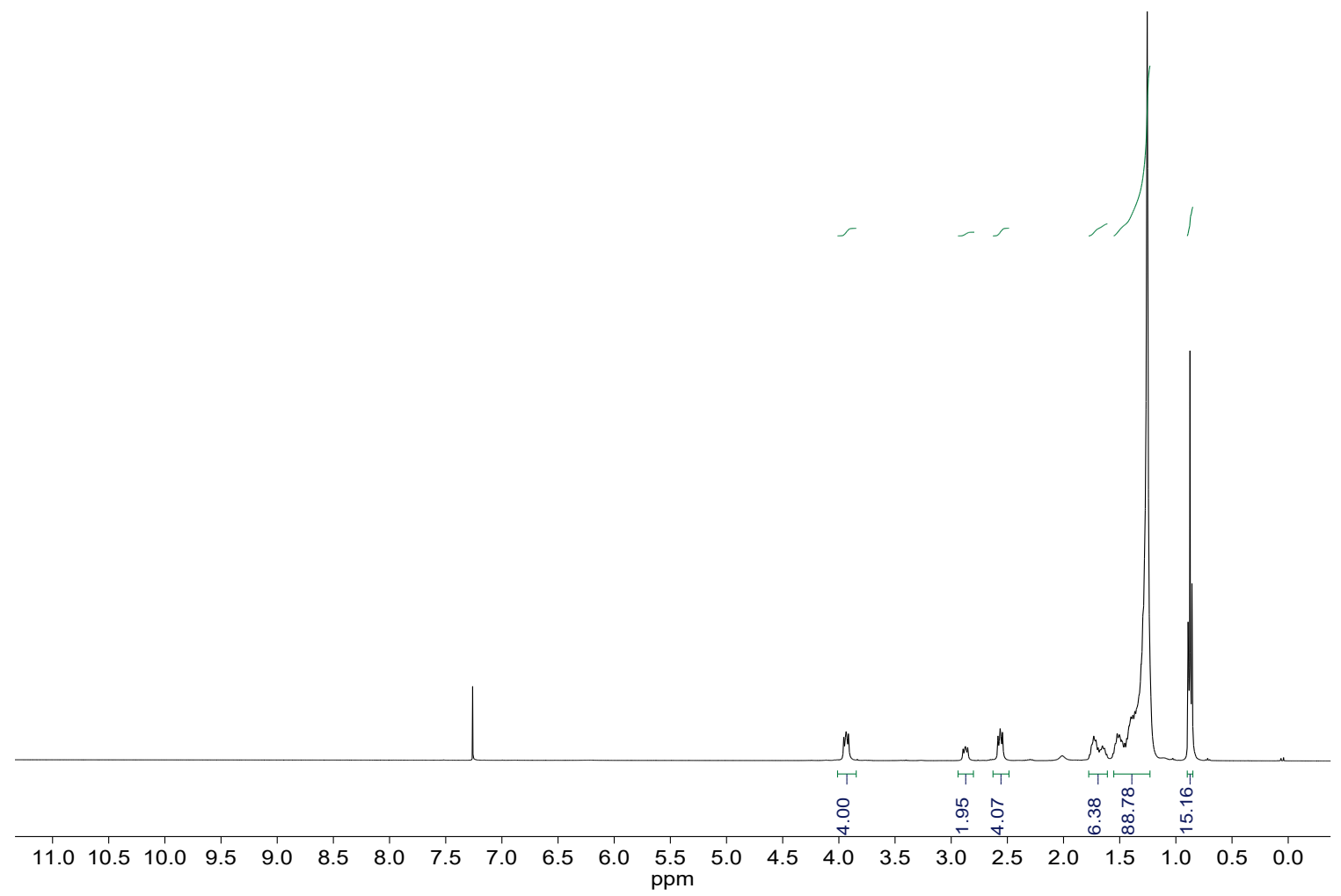

Compound 5-Cp(CN) $\left.)_{5^{-1}} 11\left({ }^{13} \mathrm{C}_{\{}{ }^{1} \mathrm{H}\right\}, 101 \mathrm{MHz}, \mathrm{CDCl} l_{3}\right)$

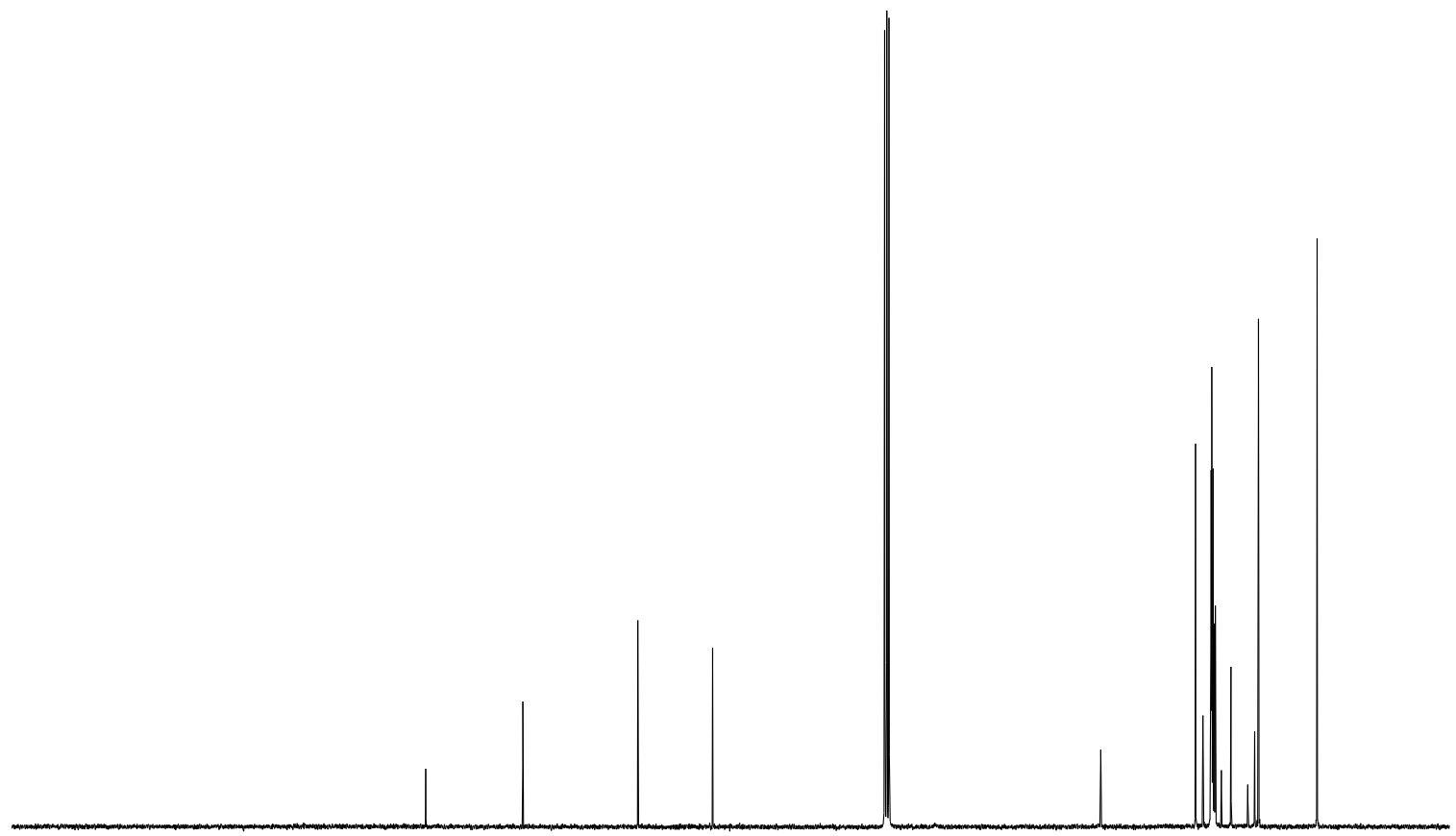

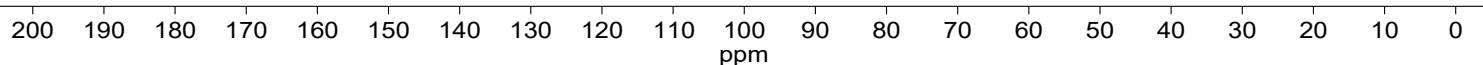


Compound 5-Cp(CN) $)^{-15}\left({ }^{1} \mathrm{H}, 400 \mathrm{MHz}, \mathrm{CDCl}_{3}\right)$

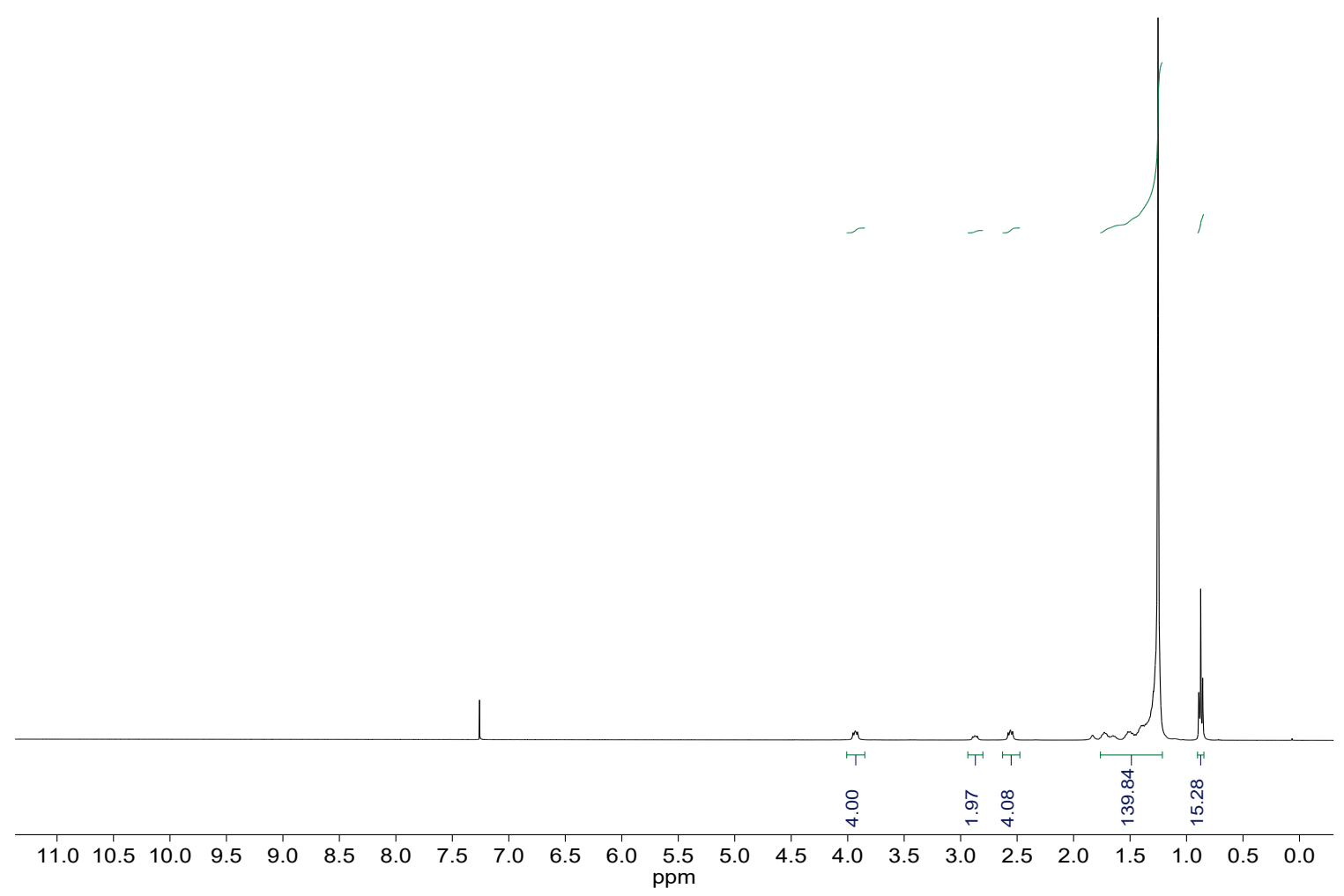

Compound 5-Cp(CN) $\left.)_{5-15}\left({ }^{13} C_{\{}{ }^{1} H\right\}, 101 \mathrm{MHz}, C D C l_{3}\right)$

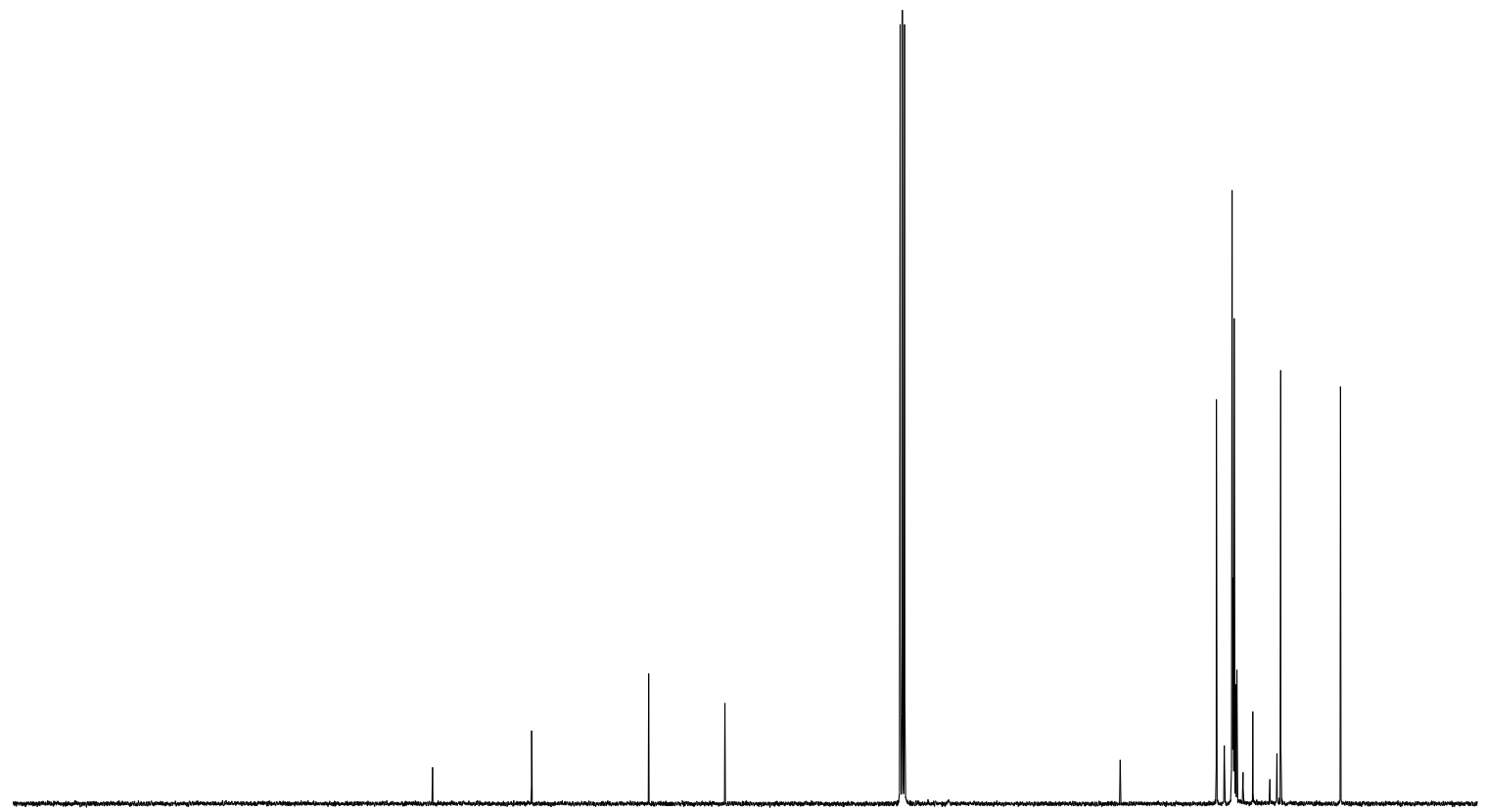

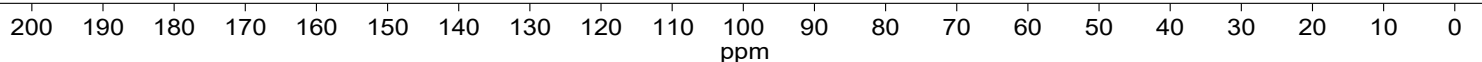




\subsubsection{ATR-FT-IR spectra}

Compounds 5-X-1 $\left(\mathrm{X}=\mathrm{I}^{-},\left[\mathrm{NO}_{3}\right]^{-},\left[\mathrm{Cp}(\mathrm{CN})_{5}\right]^{-} ; \mathrm{Ge}-\mathrm{ATR}\right)$

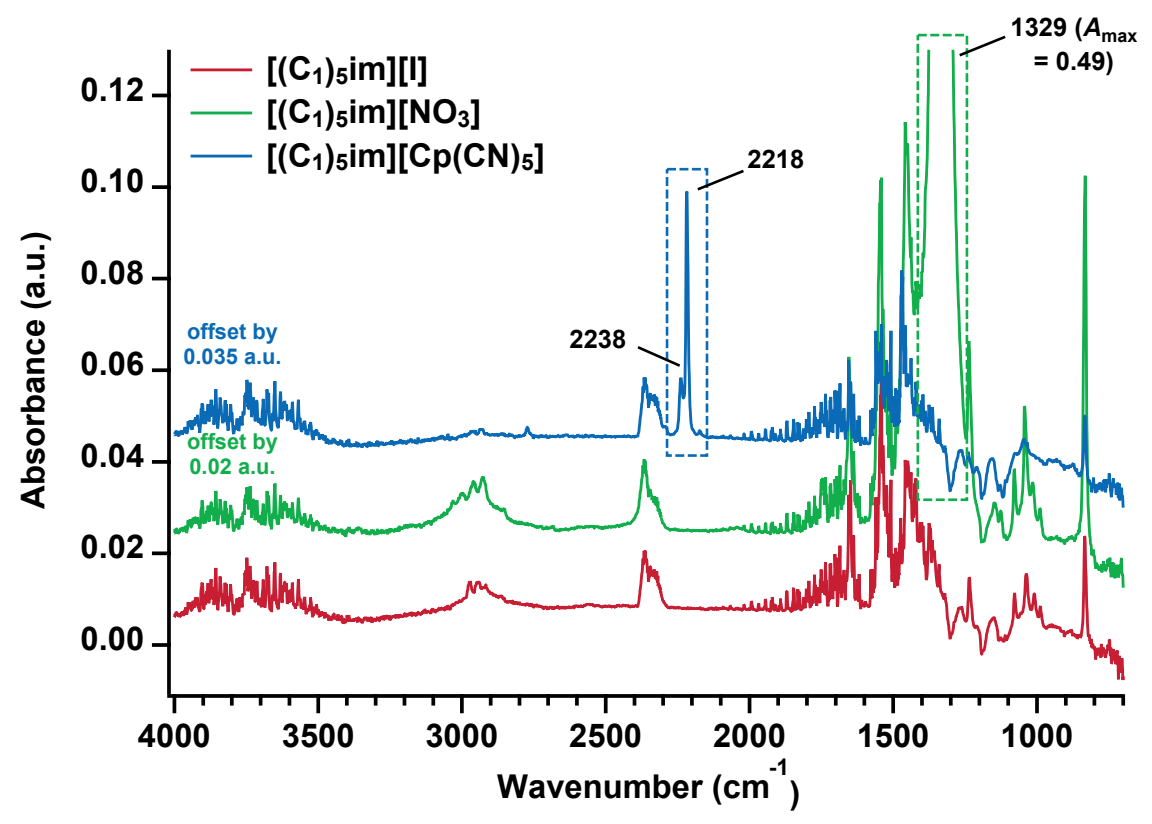

Compounds 5-X-7 ( $\left.\mathrm{X}=\mathrm{Br}^{-},\left[\mathrm{NO}_{3}\right]^{-},\left[\mathrm{Cp}(\mathrm{CN})_{5}\right]^{-} ; \mathrm{Ge}-\mathrm{ATR}\right)$

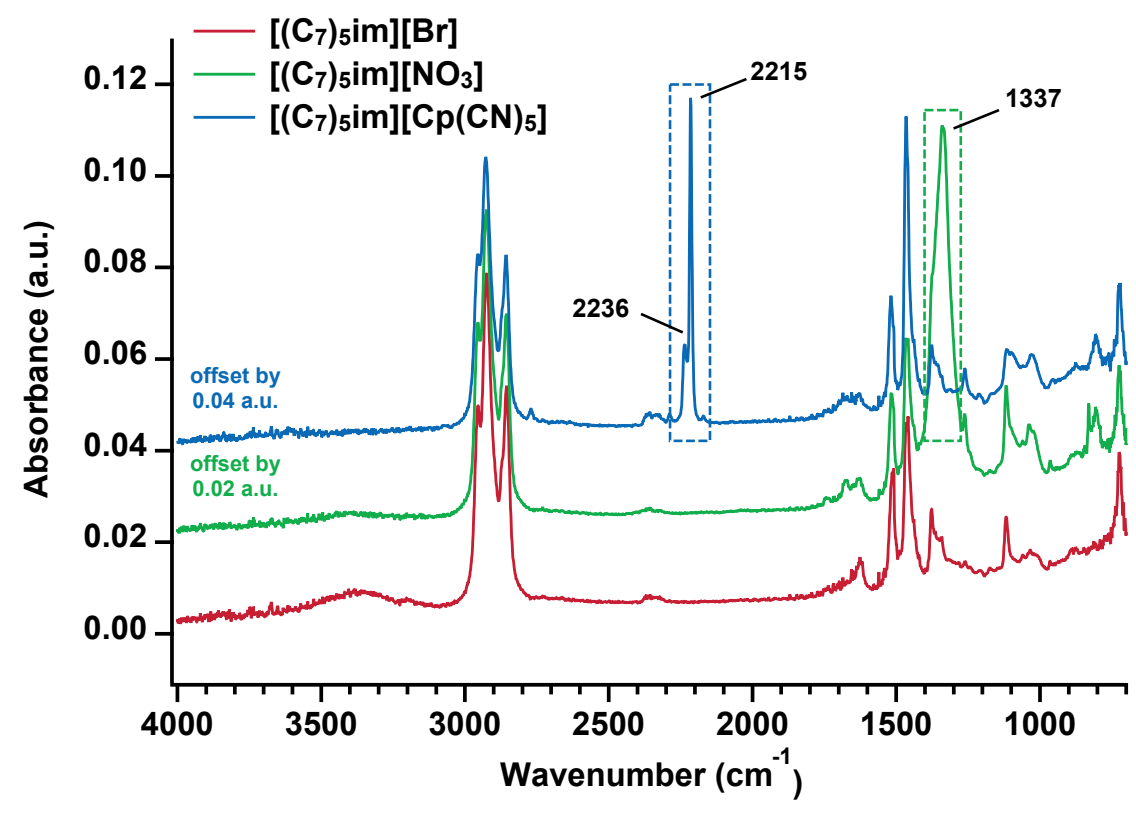


Compounds 5-X-11 ( $\mathrm{X}=\mathrm{Br}^{-},\left[\mathrm{NO}_{3}\right]^{-},\left[\mathrm{Cp}(\mathrm{CN})_{5}\right]^{-}$; Ge-ATR)

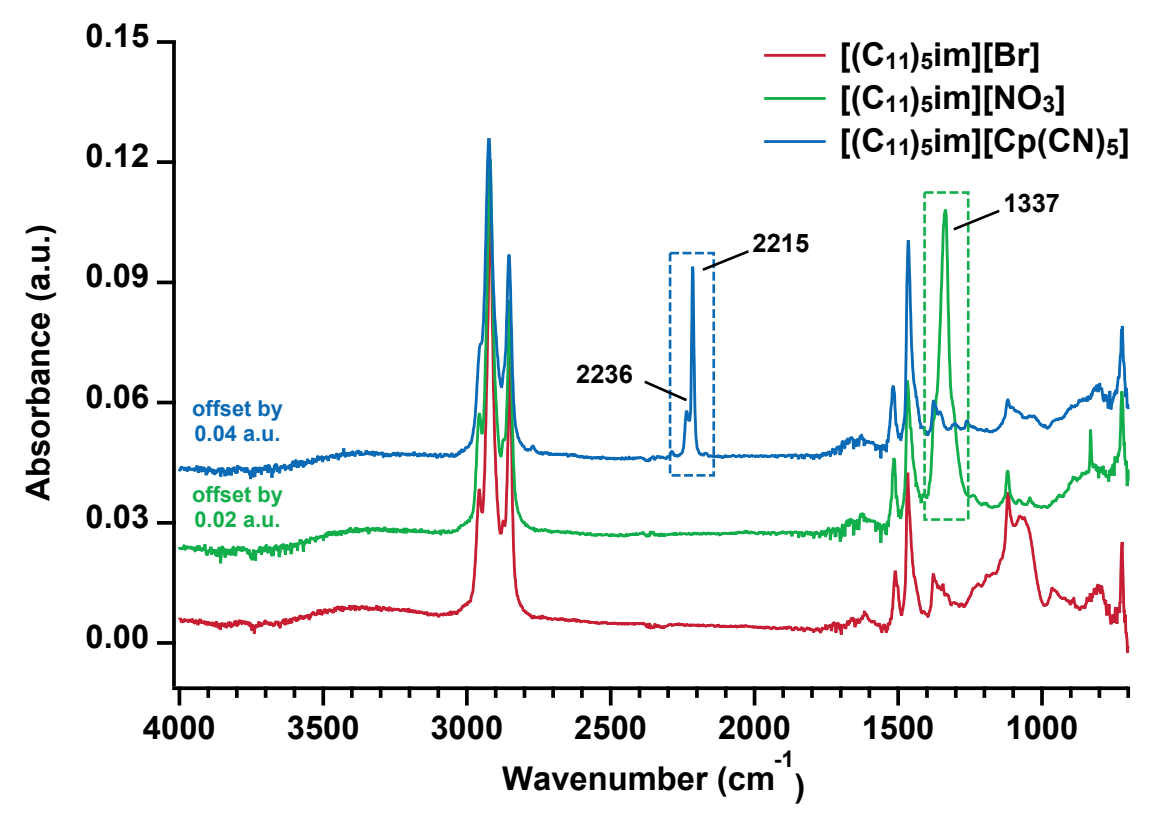

Compounds 5-X-15 ( $\mathrm{X}=\mathrm{Br}^{-},\left[\mathrm{NO}_{3}\right]^{-},\left[\mathrm{Cp}(\mathrm{CN})_{5}\right]^{-}$; Ge-ATR)

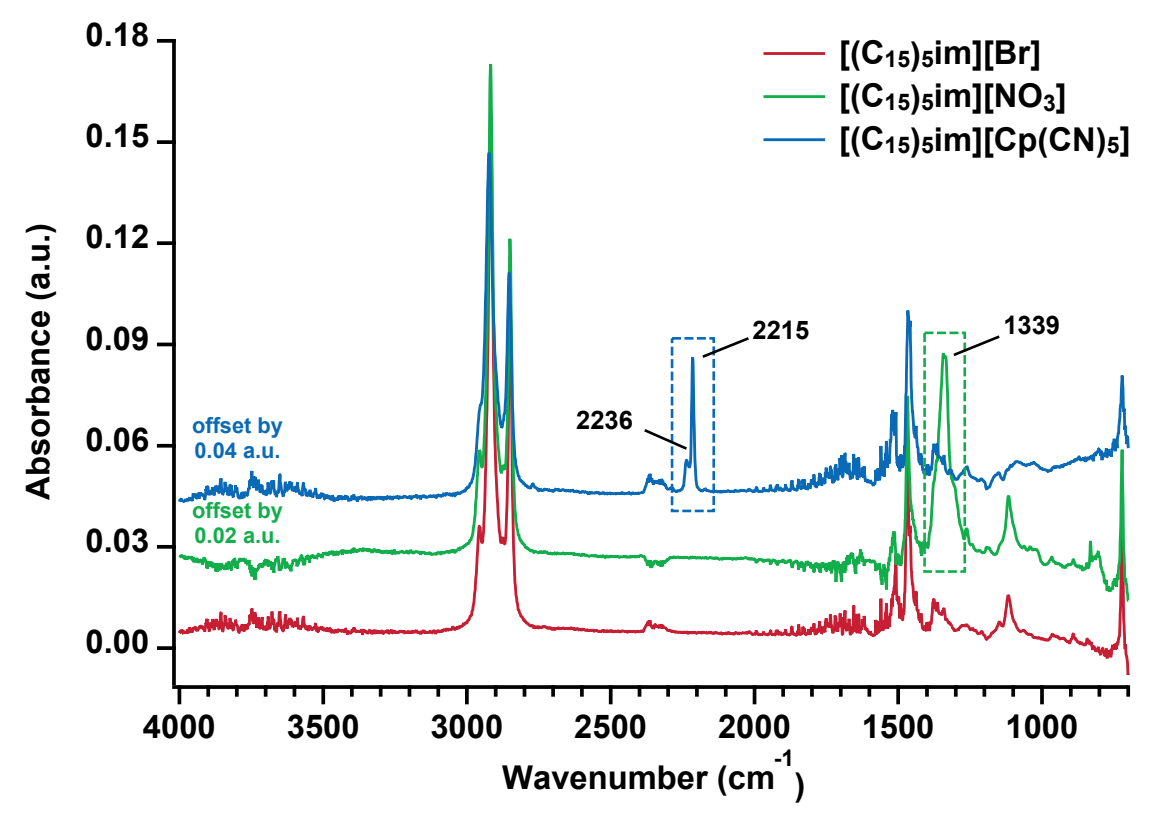


Precursor $\mathrm{Na}\left[\mathrm{Cp}(\mathrm{CN})_{5}\right]$

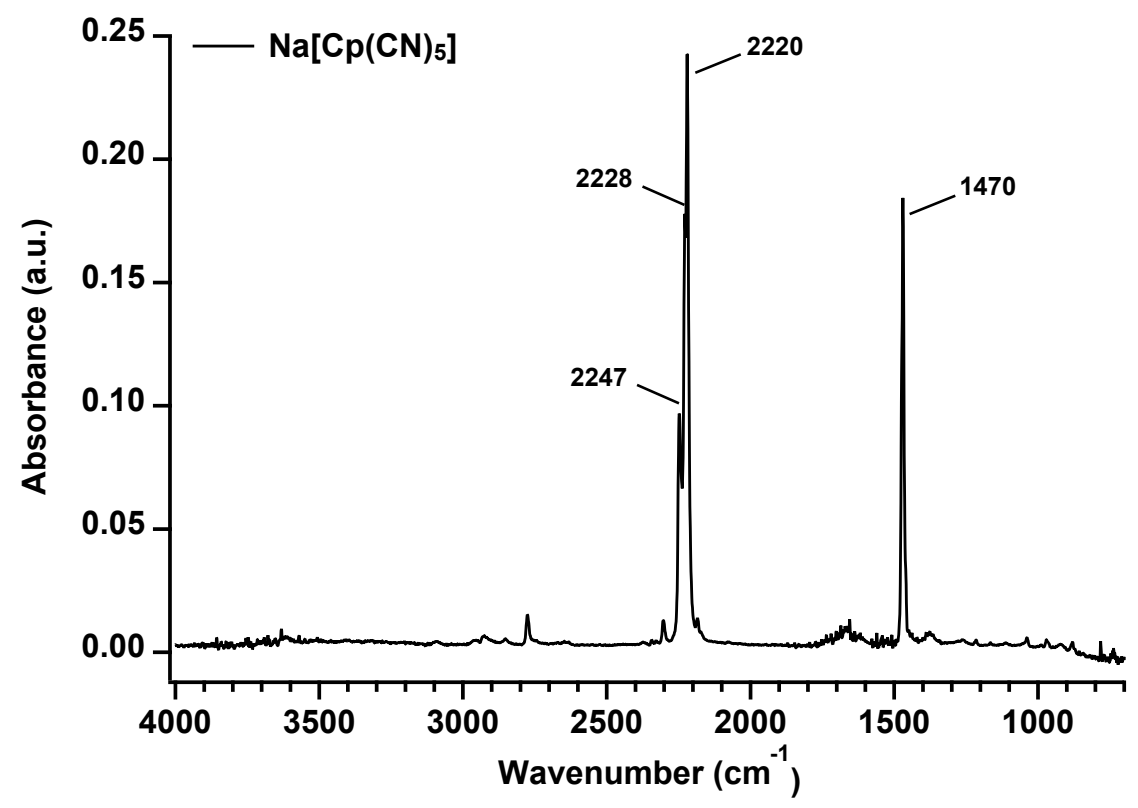




\section{Polarized-light optical microscopy (POM) data}

Note: All images were recorded using crossed polarizers.

(a)

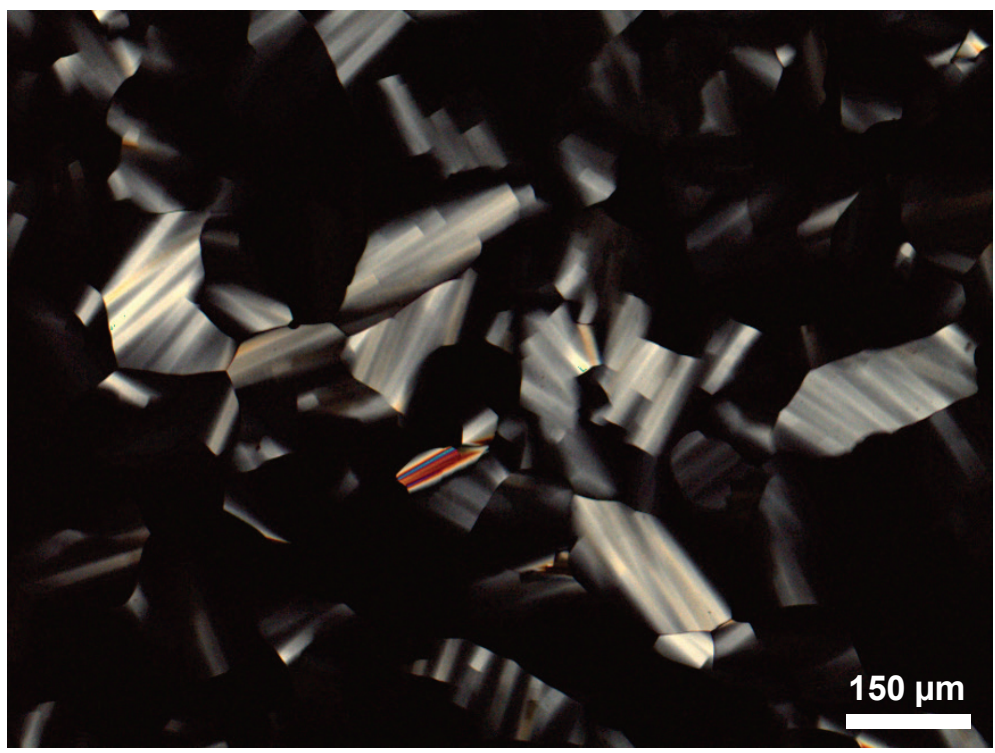

(b)

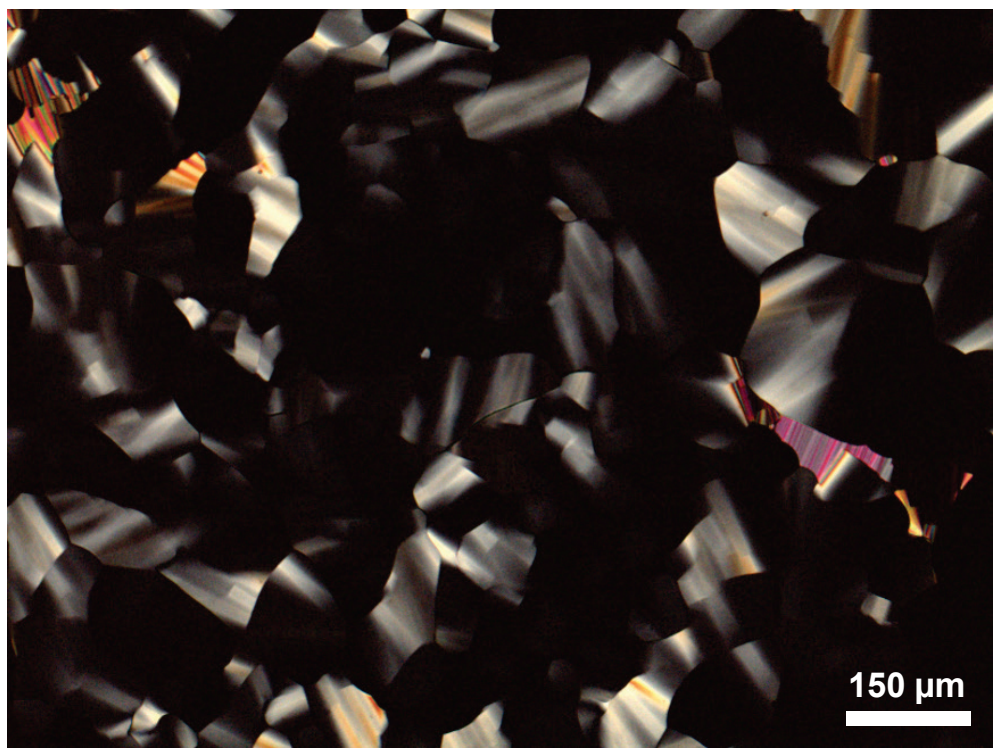

Figure S1. POM images of the $\mathrm{M}_{\text {hex }}$ phase of $5-\mathbf{C p}(\mathbf{C N})_{5}-1$ at $238^{\circ} \mathrm{C}$. 
(a)

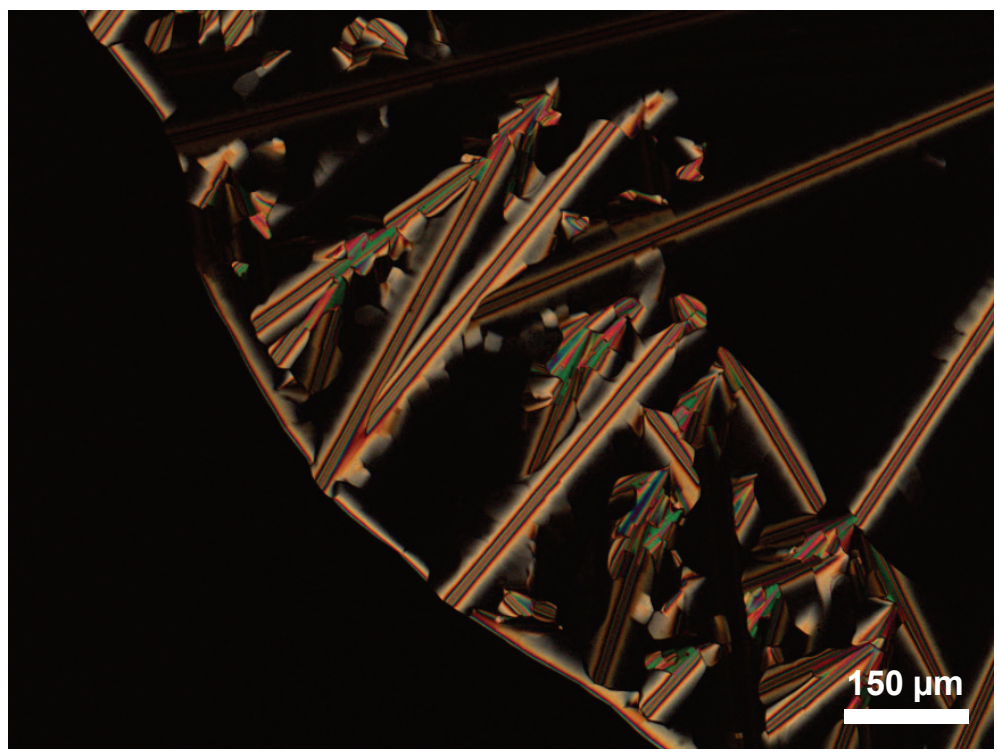

(b)

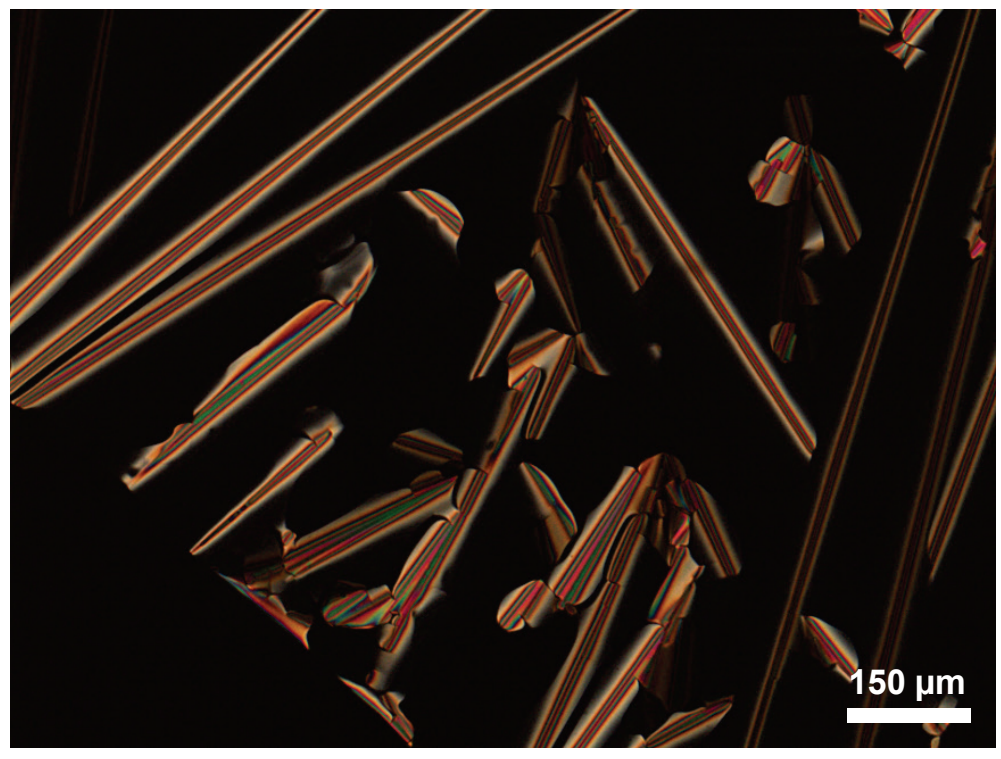

Figure S2. POM images of the $\mathrm{M}_{\text {hex }}$ phase of 5-Cp(CN) $)_{5}-\mathbf{1}$ at $239^{\circ} \mathrm{C}$. 


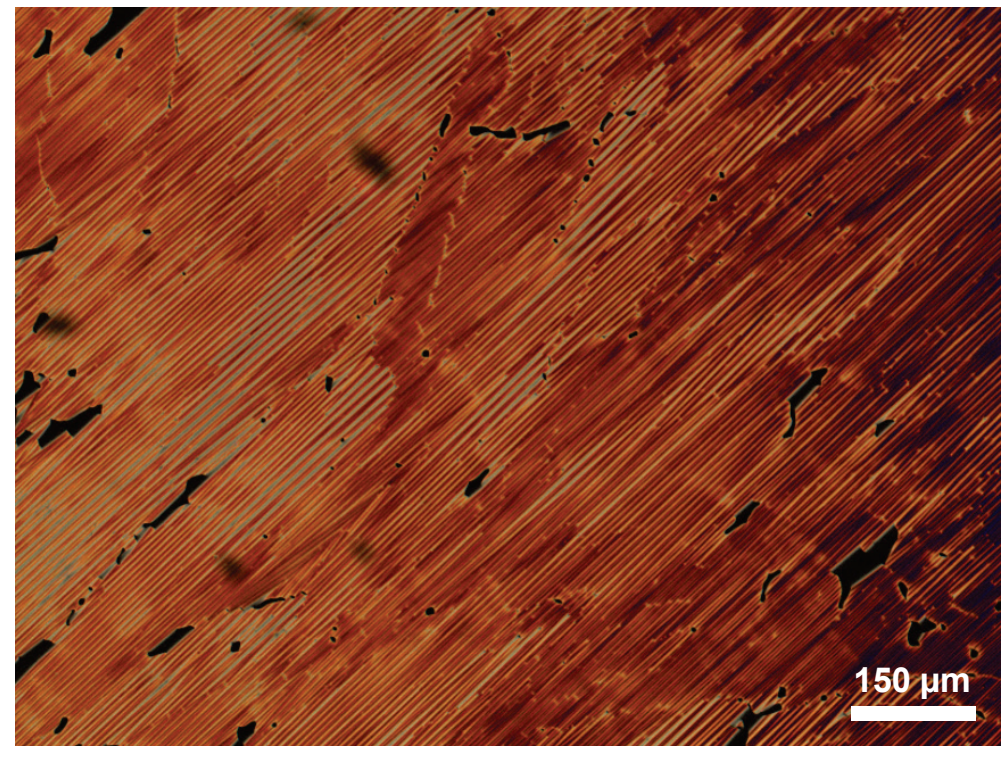

Figure S3. POM image of the $\mathrm{M}_{\text {hex }}$ phase of $\mathbf{5 - C p}(\mathbf{C N})_{5}-1$ at $238{ }^{\circ} \mathrm{C}$ as observed upon cooling from its isotropic liquid state and after pressing the sample with a needle. 

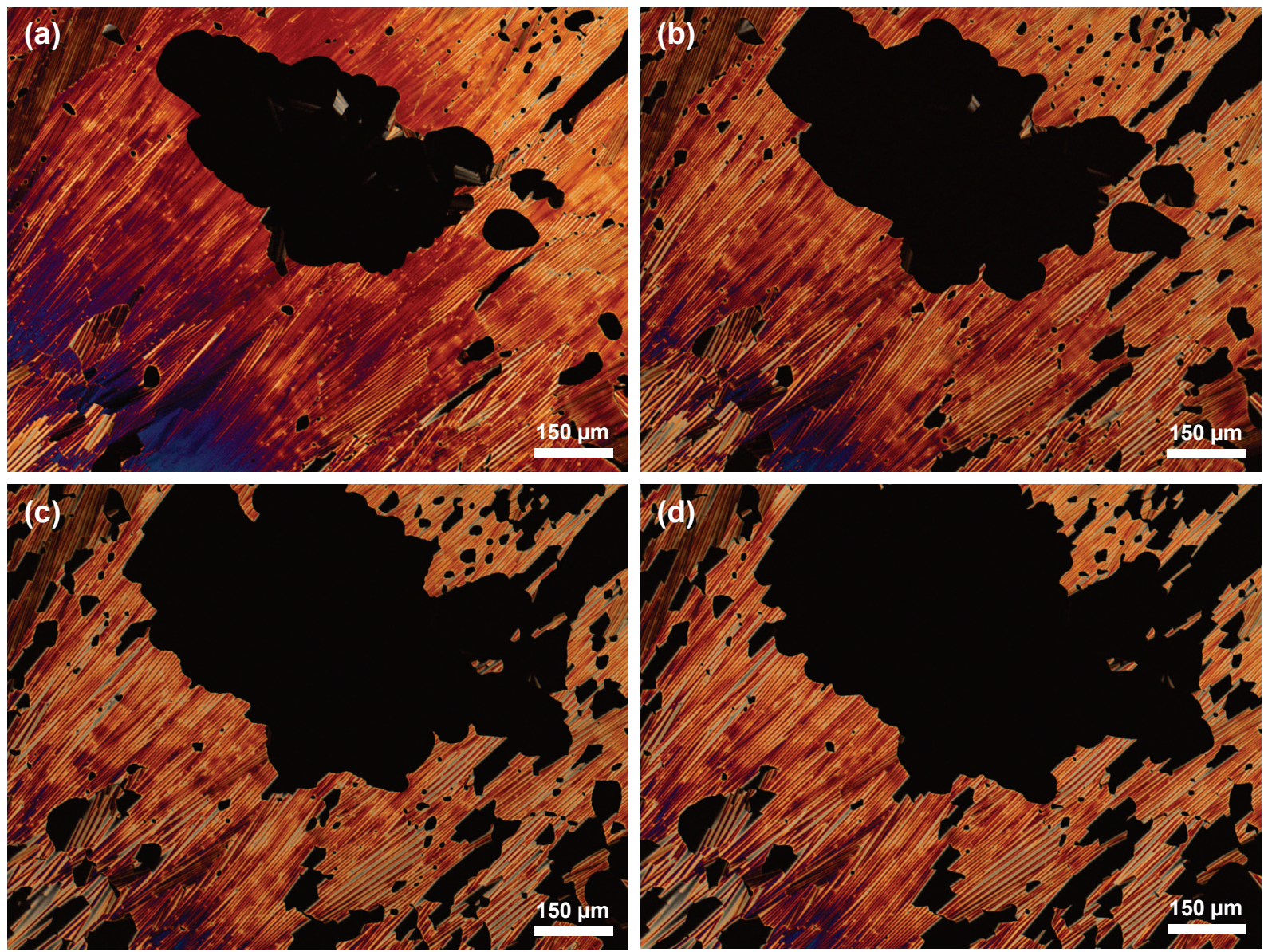

Figure S4. POM images of the $\mathrm{M}_{\text {hex }}$ phase of $\mathbf{5 - C p}(\mathbf{C N})_{5}-\mathbf{1}$ at $239{ }^{\circ} \mathrm{C}$. The picture in (a) was taken immediately after pressing the sample with a needle. Pictures (b)-(d) show the evolution of the texture with time (40 s) when no pressure was applied to the sample (the same sample area is shown in all of the images). 

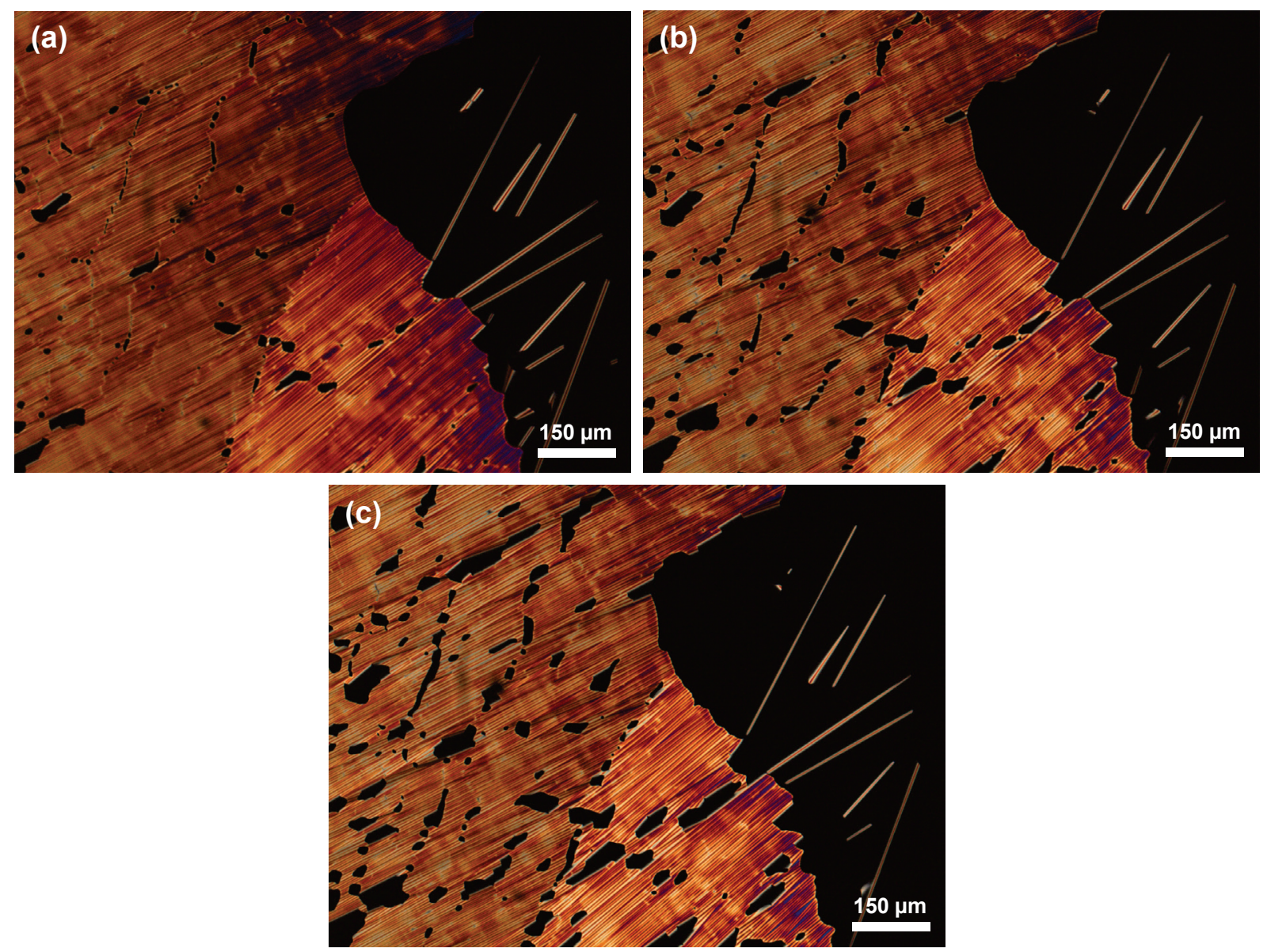

Figure S5. POM images of the $\mathrm{M}_{\text {hex }}$ phase of $\mathbf{5 - C p}(\mathbf{C N})_{5}-\mathbf{1}$ at $238{ }^{\circ} \mathrm{C}$ as observed upon cooling from its isotropic liquid state. The picture in (a) was taken immediately after pressing the sample with a needle. Pictures (b)-(c) show the evolution of the texture with time (20 s) when no pressure was applied to the sample (the same sample area is shown in all of the images). 
(a)

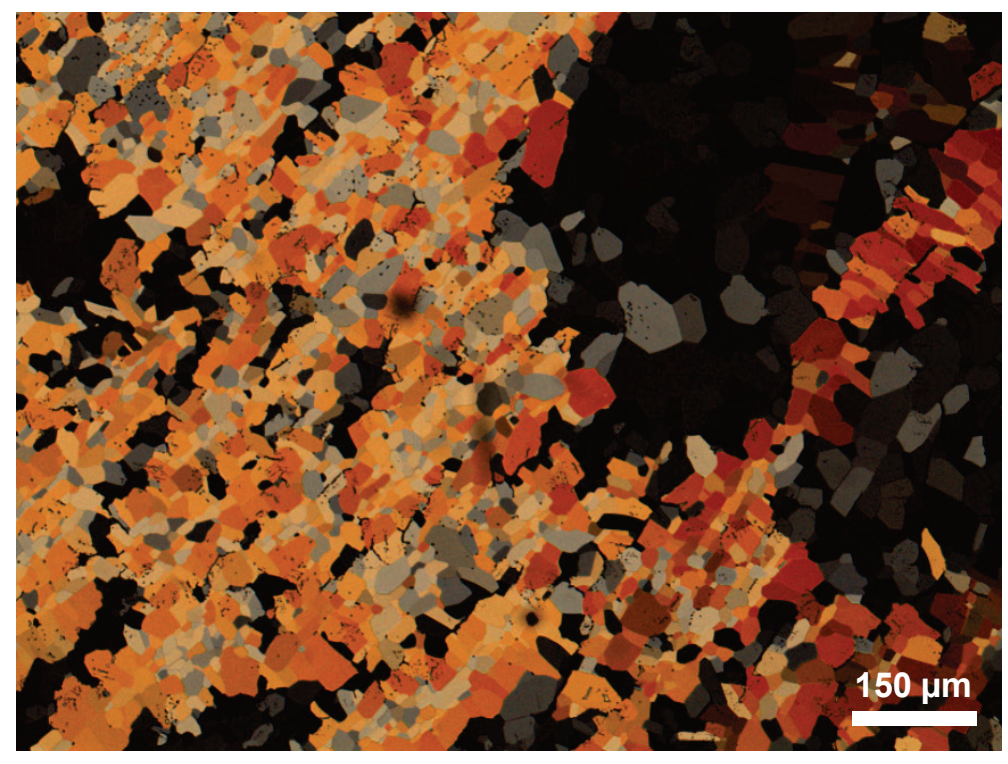

(b)

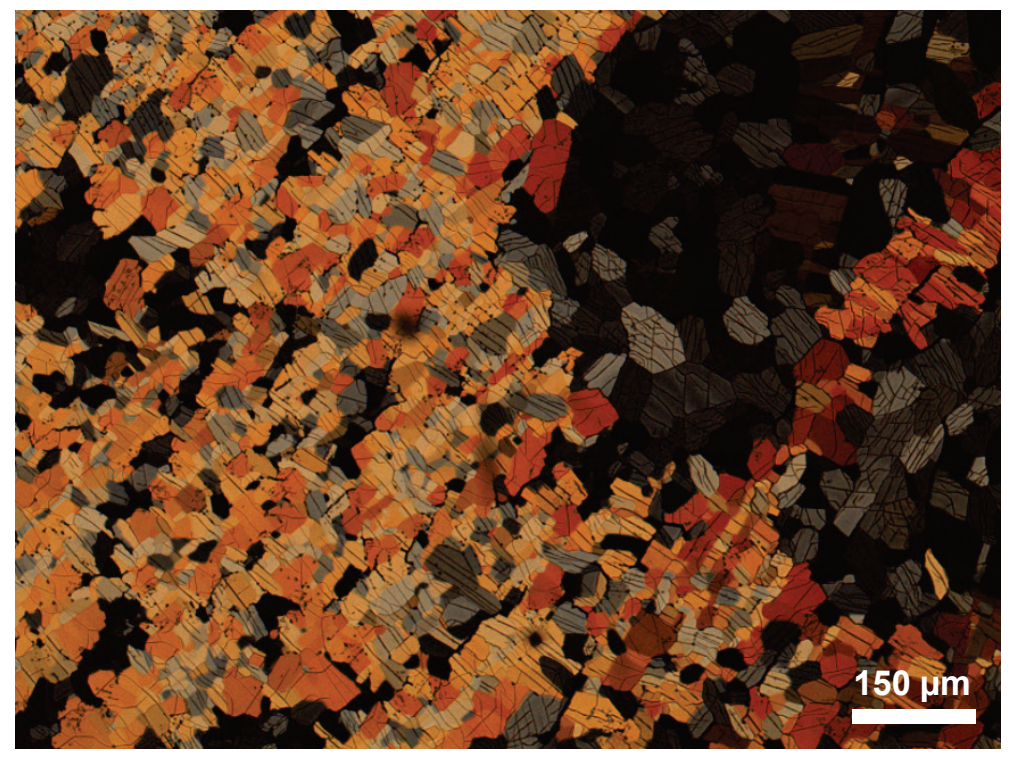

Figure S6. POM images of 5-Cp(CN) $)_{5}-1$ as observed upon cooling from its $\mathrm{M}_{\text {hex }}$ phase: (a) $\mathrm{Cr}_{2}$ phase at 206 ${ }^{\circ} \mathrm{C}$; (b) $\mathrm{Cr}_{1}$ phase at $45{ }^{\circ} \mathrm{C}$ (the same sample area is shown in both images and the textures were obtained without applying pressure to the sample). 
(a)

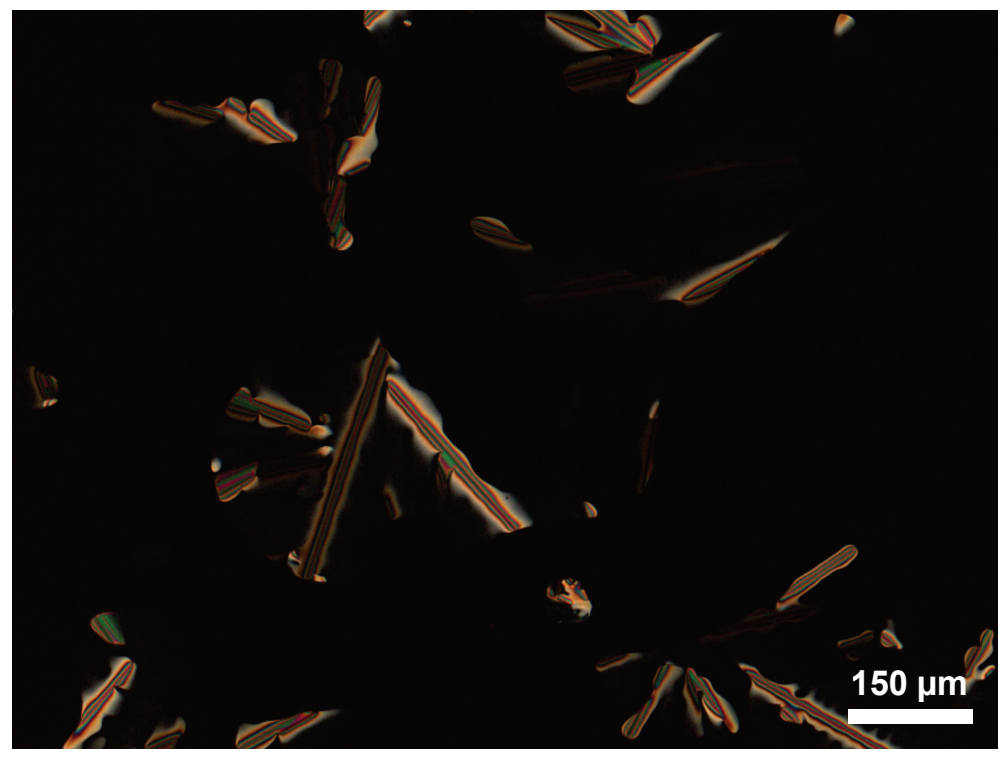

(b)

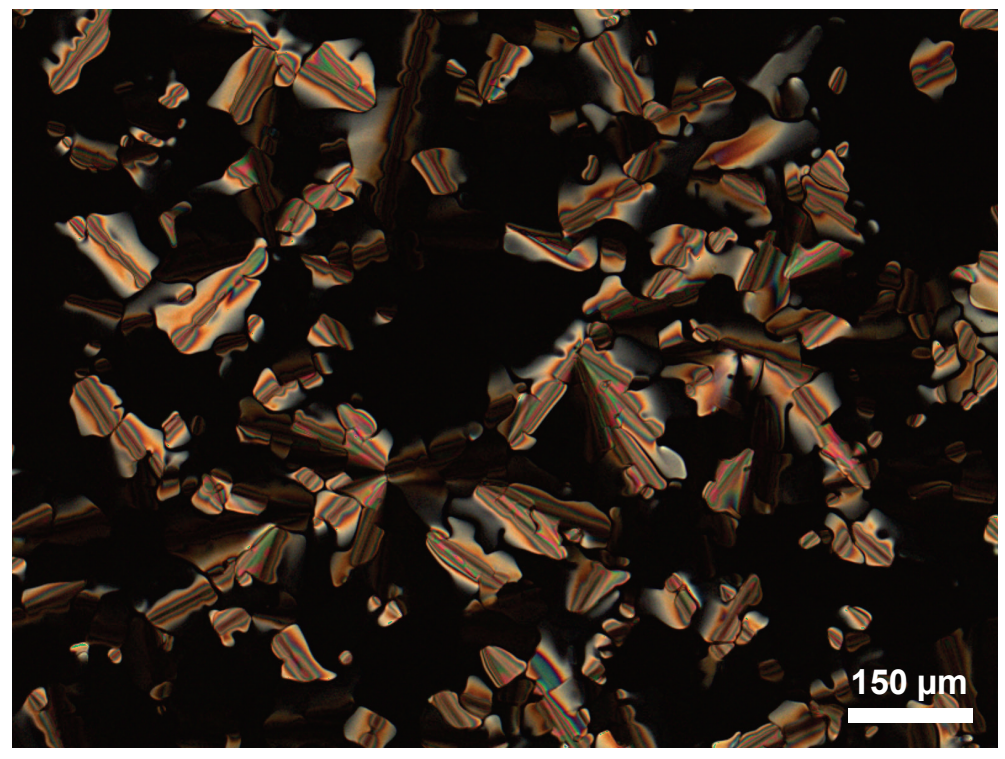

Figure S7. (a) POM image of the OIPC phase adopted by a mixture of $\mathbf{5 - C p}(\mathbf{C N})_{5}-\mathbf{1}$ and $\mathrm{Na}\left[\mathrm{Cp}(\mathrm{CN})_{5}\right]($ molar ratio: $0.975: 0.025)$ at $233^{\circ} \mathrm{C}$ as observed upon cooling from its isotropic liquid state. (b) POM image of the OIPC phase adopted by a mixture of $\mathbf{5 - C p}(\mathbf{C N})_{5}-\mathbf{1}$ and $\mathrm{Na}\left[\mathrm{Cp}(\mathrm{CN})_{5}\right]\left(\right.$ molar ratio: $0.95: 0.05$ ) at $230{ }^{\circ} \mathrm{C}$ as observed upon cooling from its isotropic liquid state. 


\section{Differential scanning calorimetry (DSC) data}

\subsection{DSC thermograms}

Abbreviations used in the figures below: $\mathrm{M}_{\mathrm{hex}}=$ plastic crystal mesophase with $2 \mathrm{D}$ hexagonal symmetry; Iso = isotropic liquid phase. Tabulated DSC data can be found in Section 3.2.

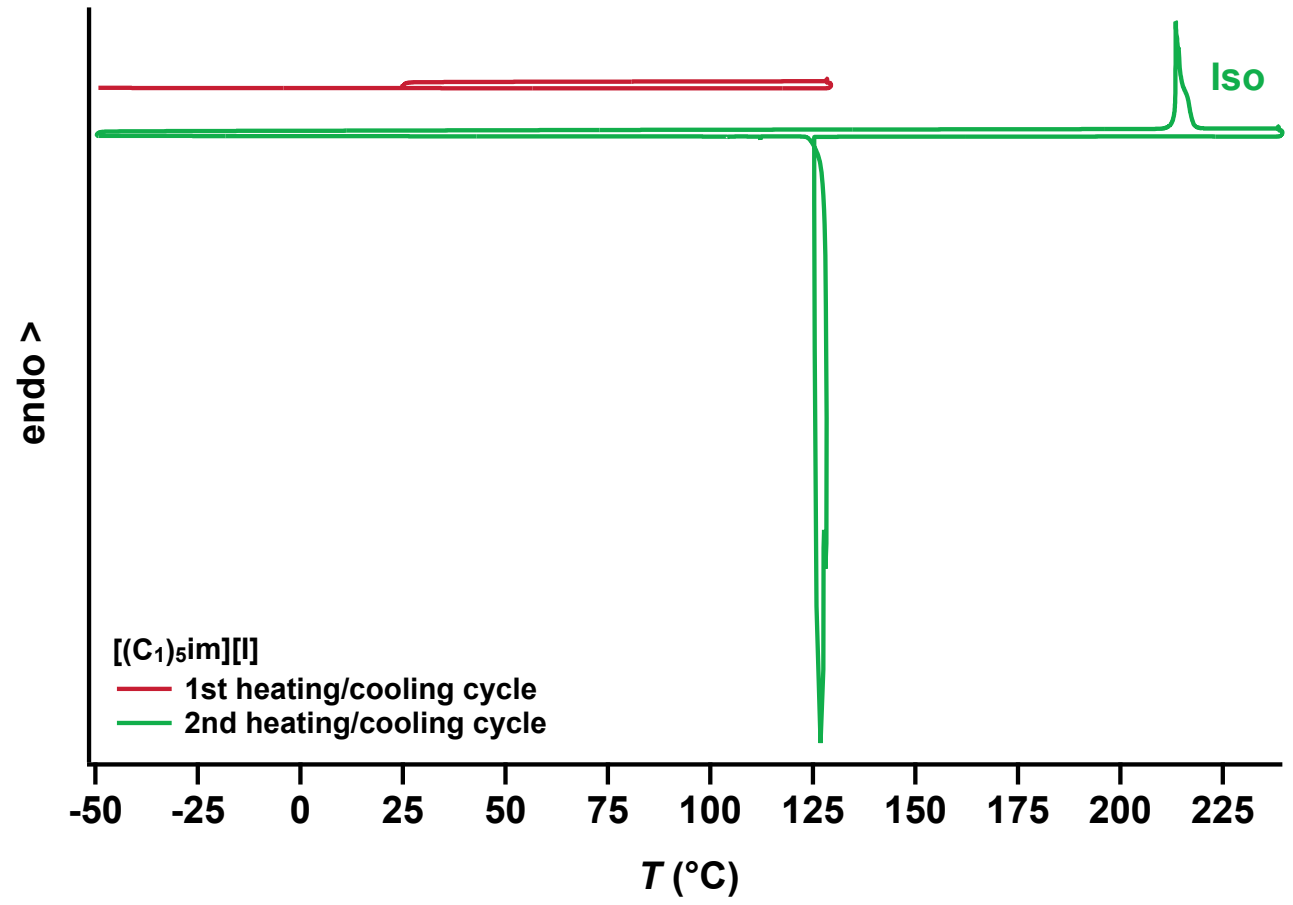

Figure S8. DSC data recorded for compound 5-I-1 at a heating rate of $10{ }^{\circ} \mathrm{C} \min ^{-1}$ and a cooling rate of $5{ }^{\circ} \mathrm{C}$ $\min ^{-1}$ under an atmosphere of $\mathrm{N}_{2}$. Endothermic peaks point upward. 


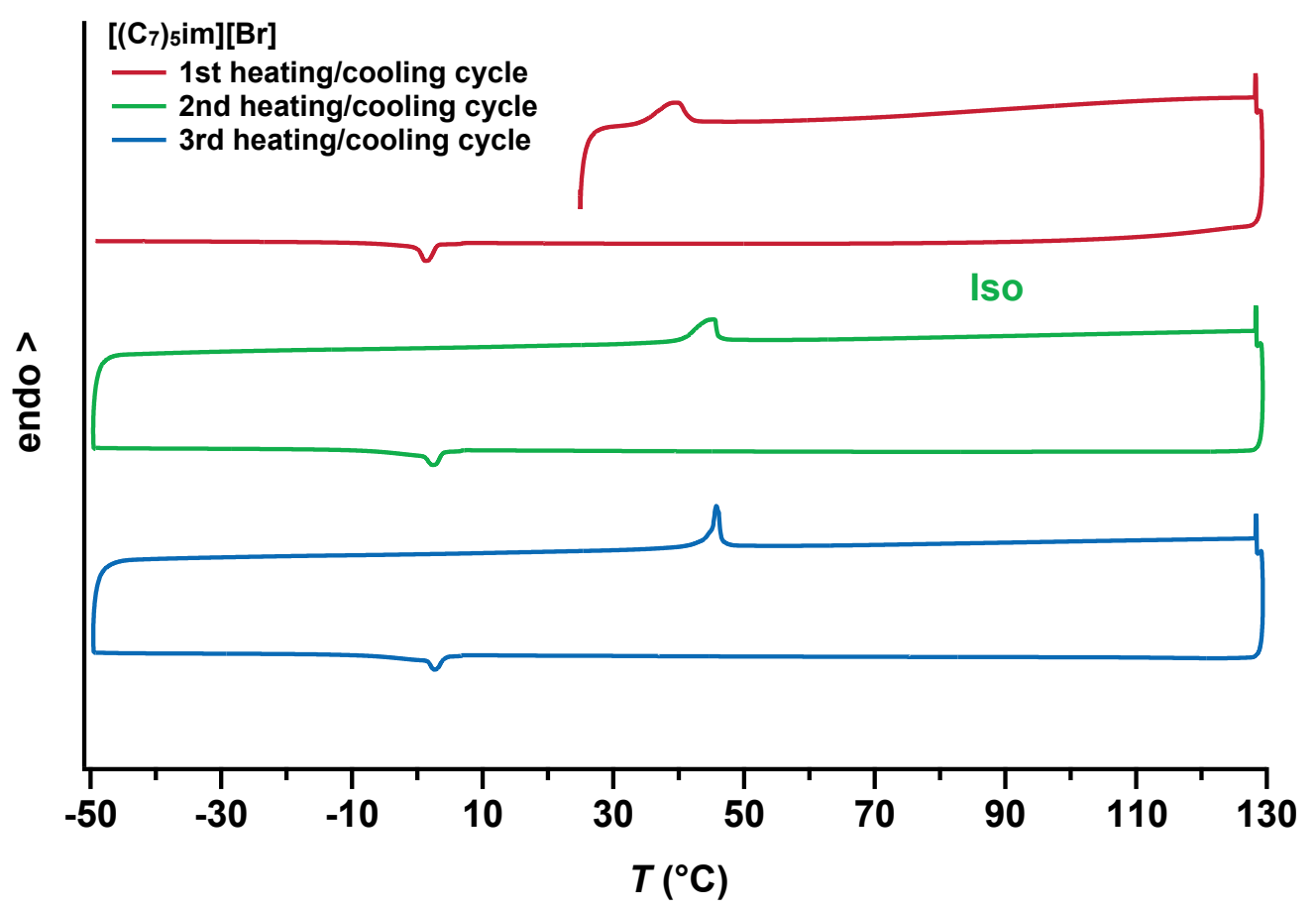

Figure S9. DSC data recorded for compound 5-Br-7 at a heating rate of $10{ }^{\circ} \mathrm{C} \mathrm{min}^{-1}$ and a cooling rate of 5 ${ }^{\circ} \mathrm{C} \min ^{-1}$ under an atmosphere of $\mathrm{N}_{2}$. Endothermic peaks point upward.

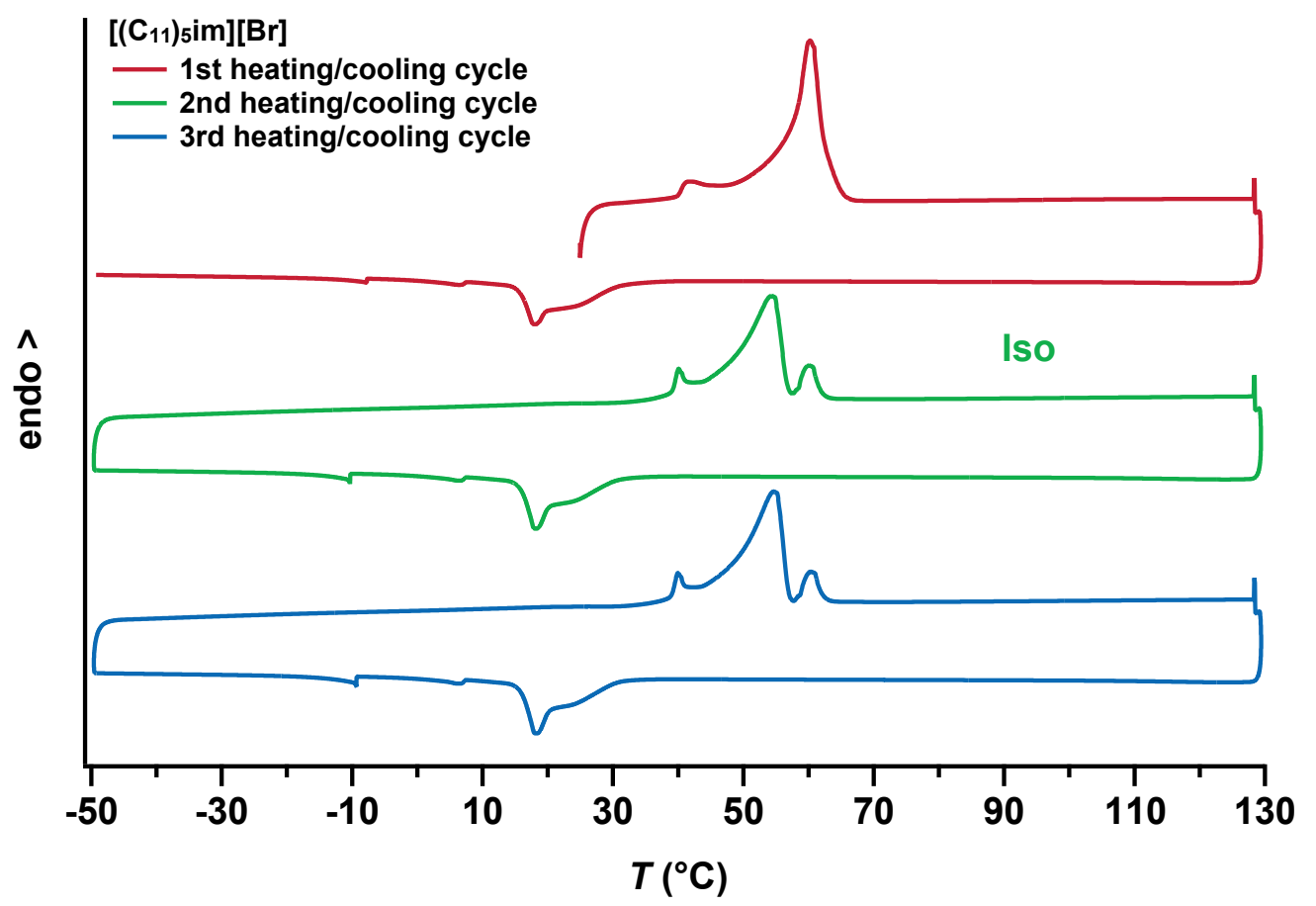

Figure S10. DSC data recorded for compound 5-Br-11 at a heating rate of $10{ }^{\circ} \mathrm{C} \mathrm{min}^{-1}$ and a cooling rate of 5 ${ }^{\circ} \mathrm{C} \min ^{-1}$ under an atmosphere of $\mathrm{N}_{2}$. Endothermic peaks point upward. 


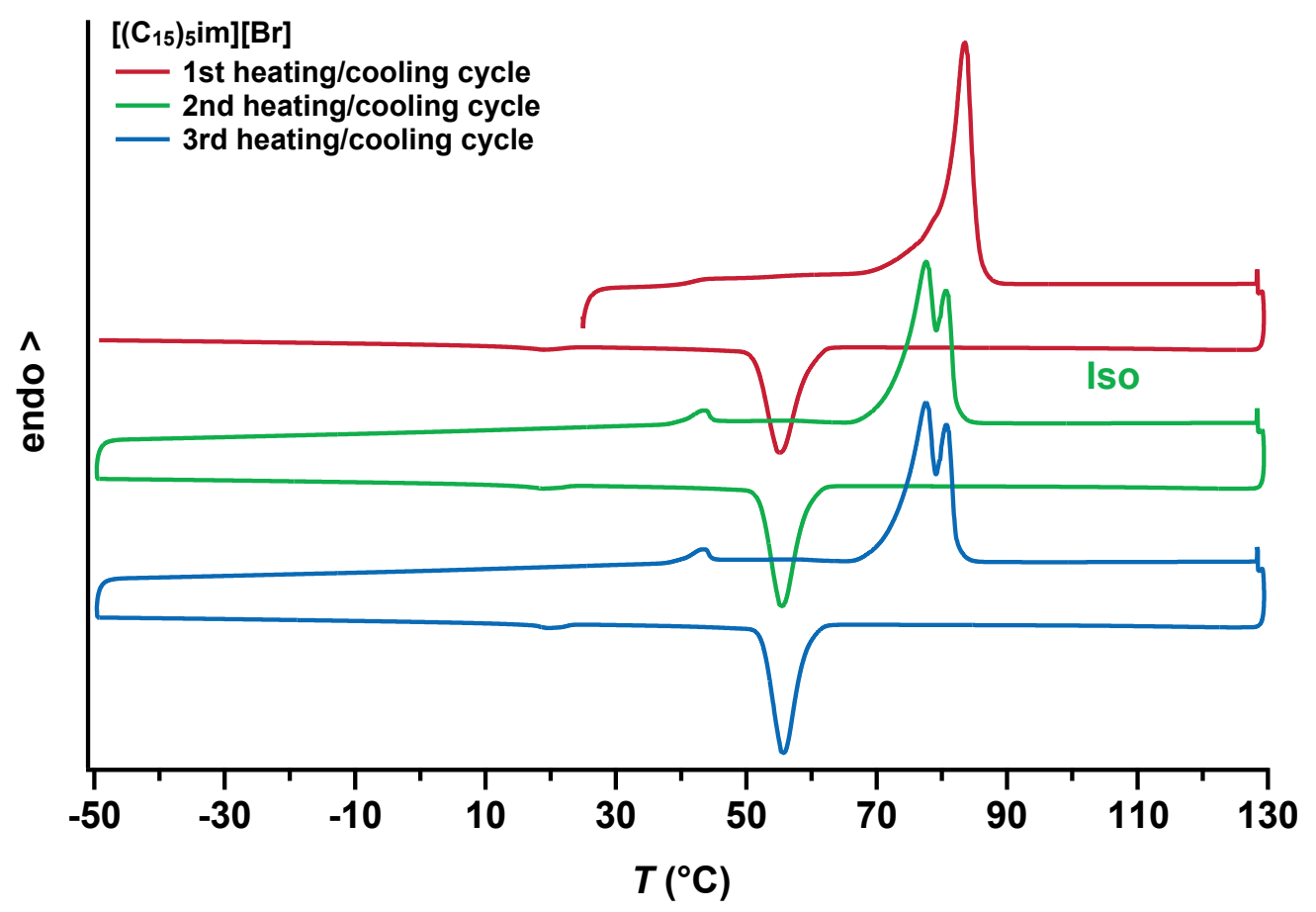

Figure S11. DSC data recorded for compound 5-Br-15 at a heating rate of $10{ }^{\circ} \mathrm{C} \min ^{-1}$ and a cooling rate of 5 ${ }^{\circ} \mathrm{C} \min ^{-1}$ under an atmosphere of $\mathrm{N}_{2}$. Endothermic peaks point upward.

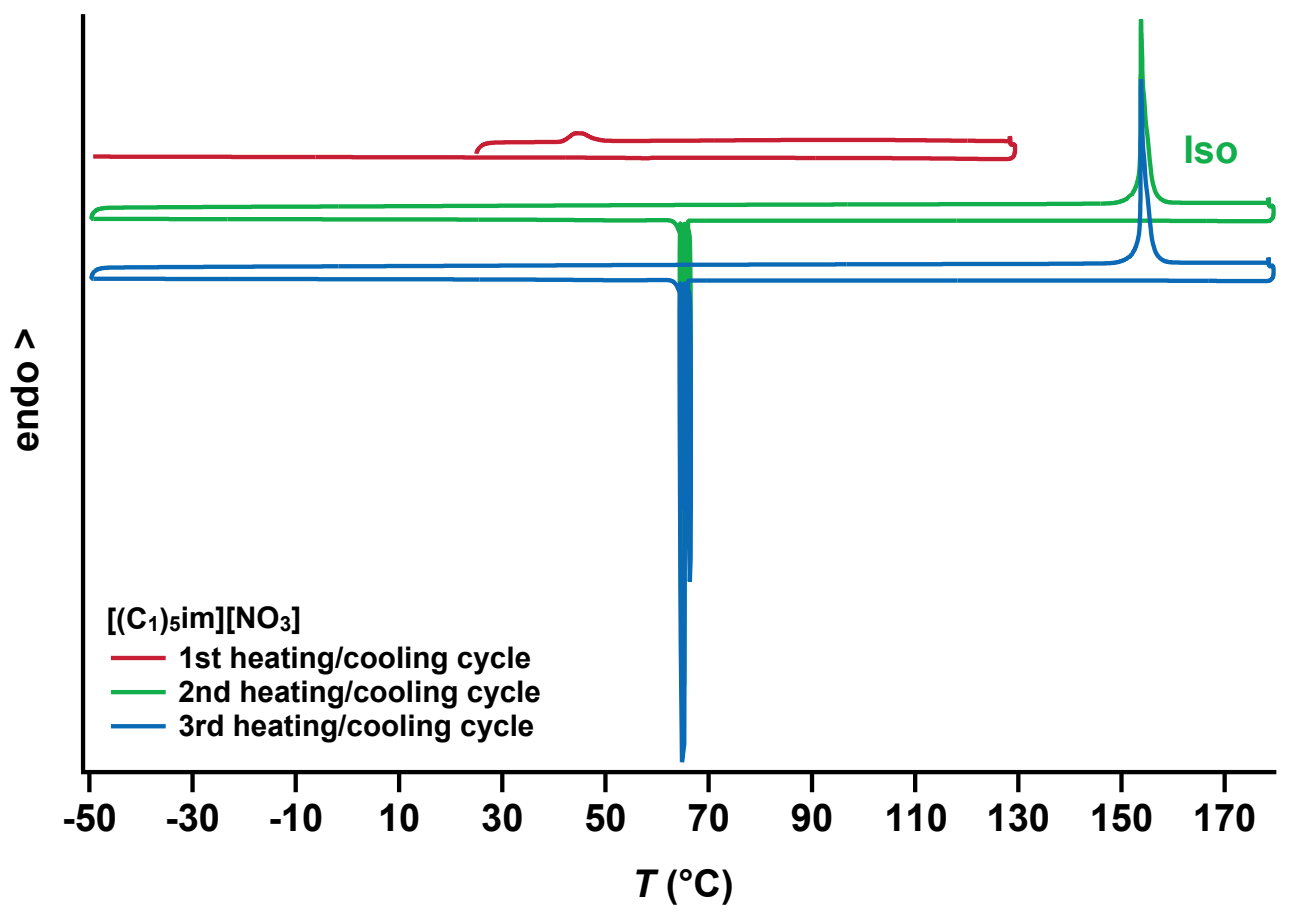

Figure S12. DSC data recorded for compound 5- $\mathrm{NO}_{3}-\mathbf{1}$ at a heating rate of $10{ }^{\circ} \mathrm{C} \min ^{-1}$ and a cooling rate of $5{ }^{\circ} \mathrm{C} \mathrm{min}^{-1}$ under an atmosphere of $\mathrm{N}_{2}$. Endothermic peaks point upward. 


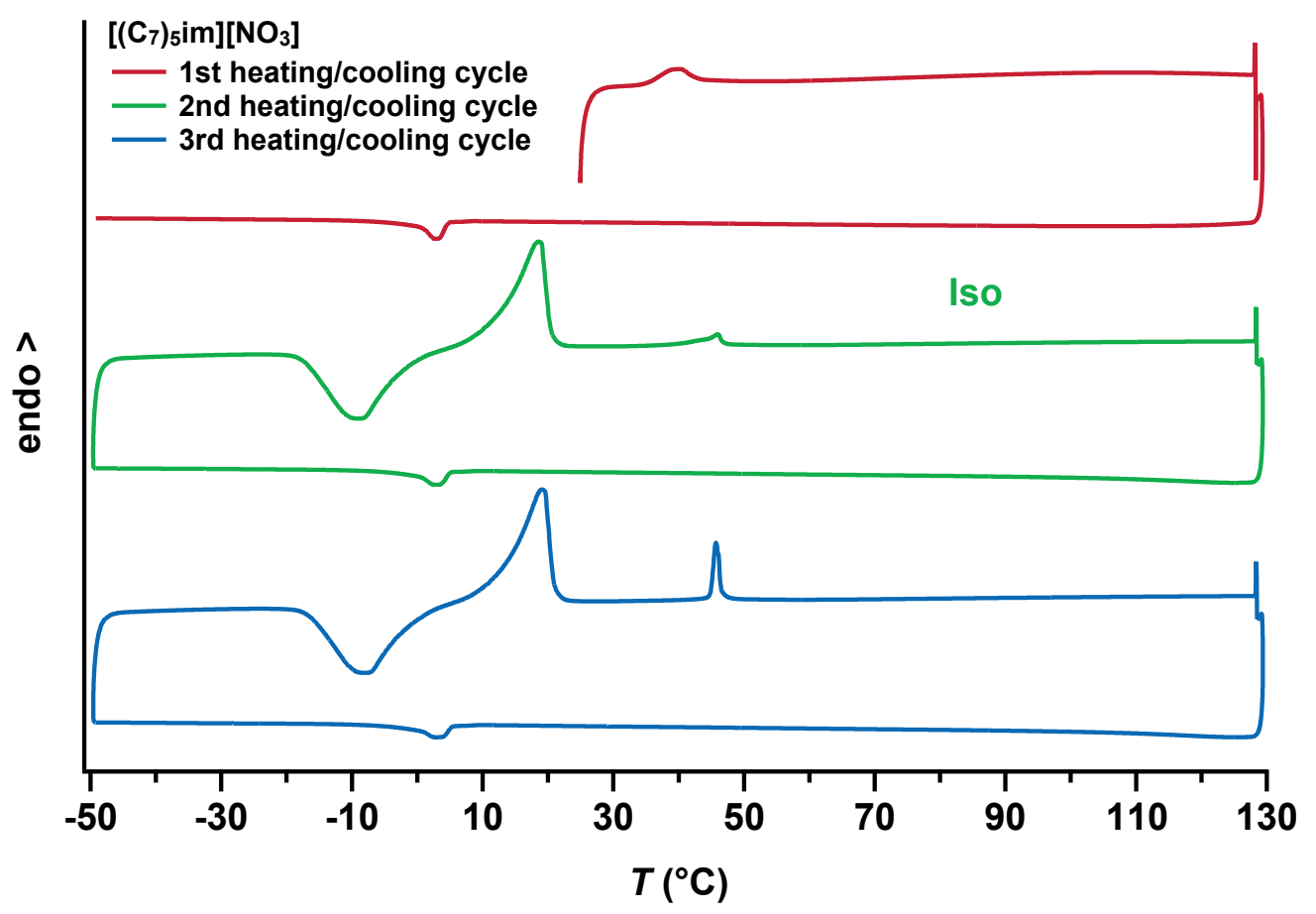

Figure S13. DSC data recorded for compound 5- $\mathrm{NO}_{3}-7$ at a heating rate of $10{ }^{\circ} \mathrm{C} \min ^{-1}$ and a cooling rate of $5{ }^{\circ} \mathrm{C} \mathrm{min}^{-1}$ under an atmosphere of $\mathrm{N}_{2}$. Endothermic peaks point upward.

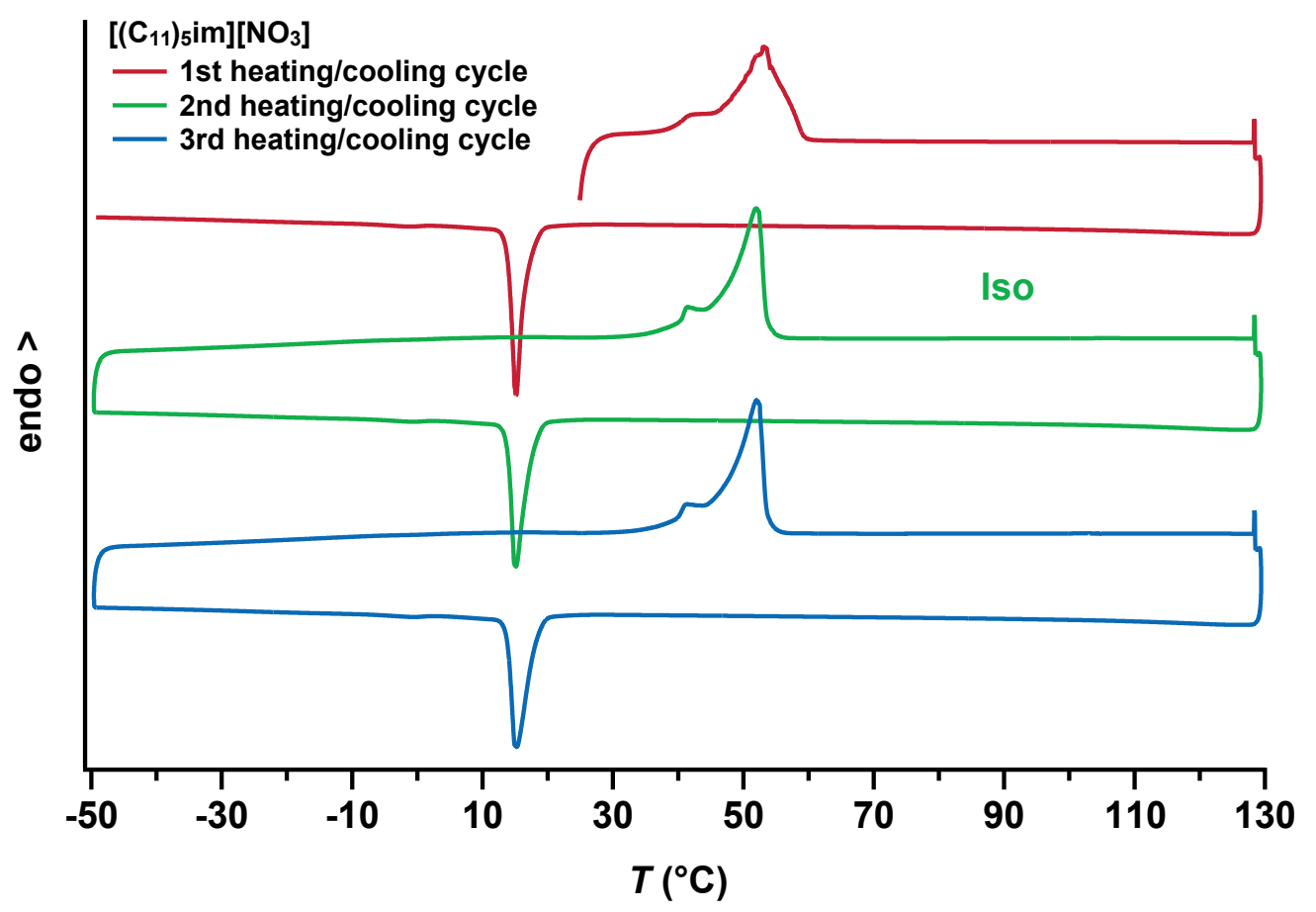

Figure S14. DSC data recorded for compound 5- $\mathrm{NO}_{3}-11$ at a heating rate of $10{ }^{\circ} \mathrm{C} \mathrm{min}^{-1}$ and a cooling rate of $5{ }^{\circ} \mathrm{C} \mathrm{min}^{-1}$ under an atmosphere of $\mathrm{N}_{2}$. Endothermic peaks point upward. 


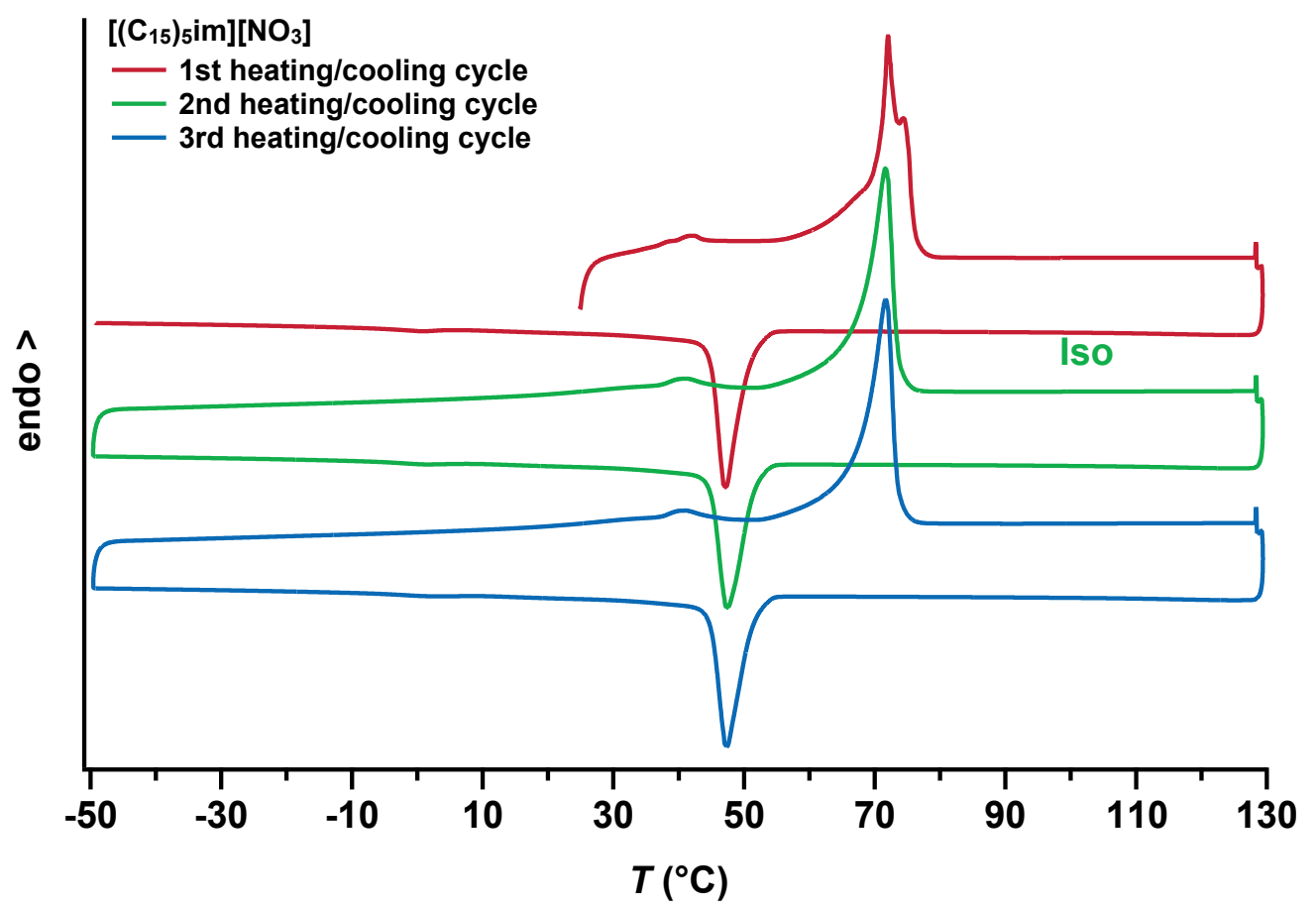

Figure S15. DSC data recorded for compound 5-NO $\mathrm{N}_{3}-15$ at a heating rate of $10{ }^{\circ} \mathrm{C} \mathrm{min}^{-1}$ and a cooling rate of $5{ }^{\circ} \mathrm{C} \mathrm{min}^{-1}$ under an atmosphere of $\mathrm{N}_{2}$. Endothermic peaks point upward.

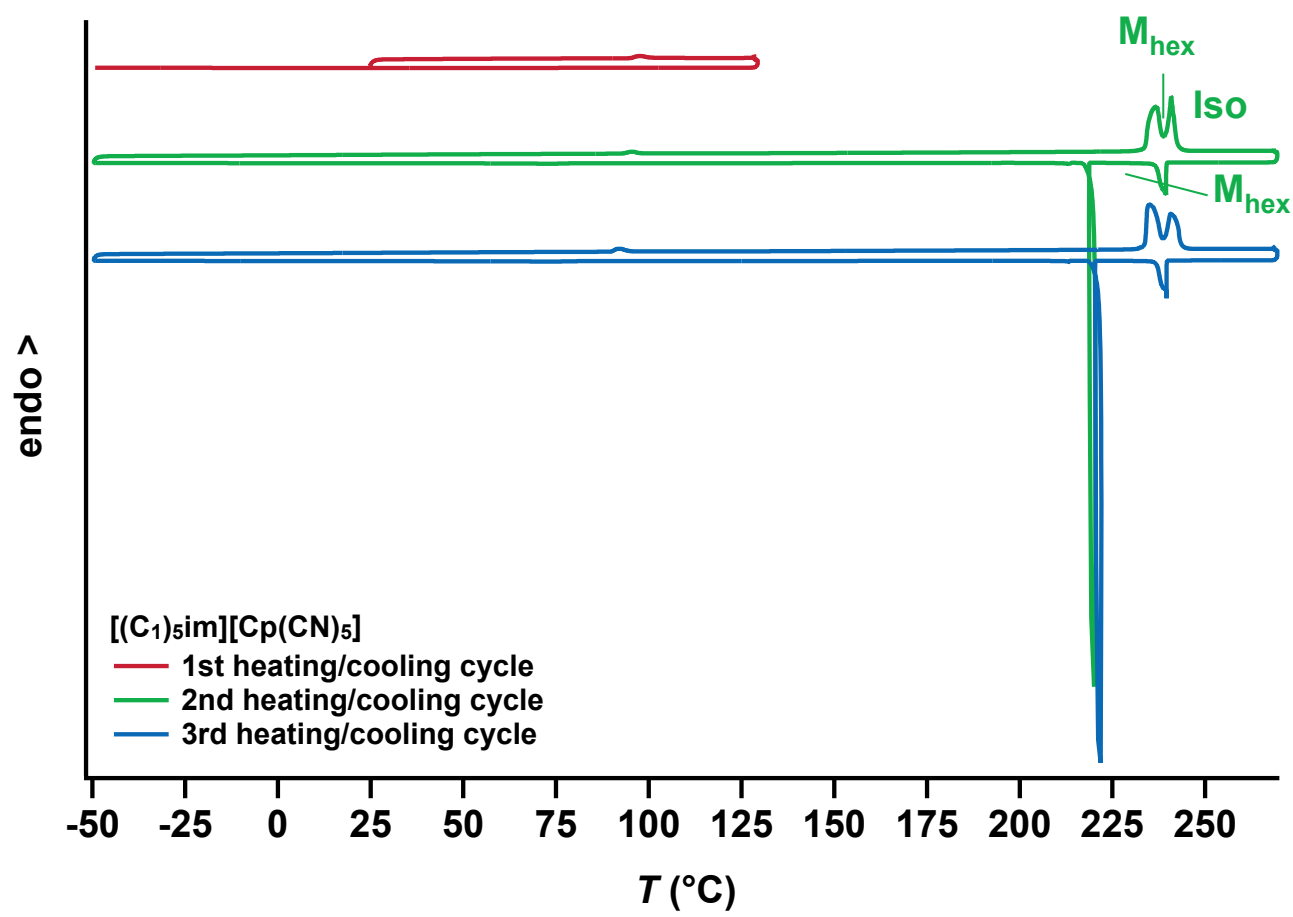

Figure S16. DSC data recorded for compound $5-\mathbf{C p}(\mathbf{C N})_{5}-1$ at a heating rate of $10{ }^{\circ} \mathrm{C} \mathrm{min}^{-1}$ and a cooling rate of $5{ }^{\circ} \mathrm{C} \mathrm{min}^{-1}$ under an atmosphere of $\mathrm{N}_{2}$. Endothermic peaks point upward. 


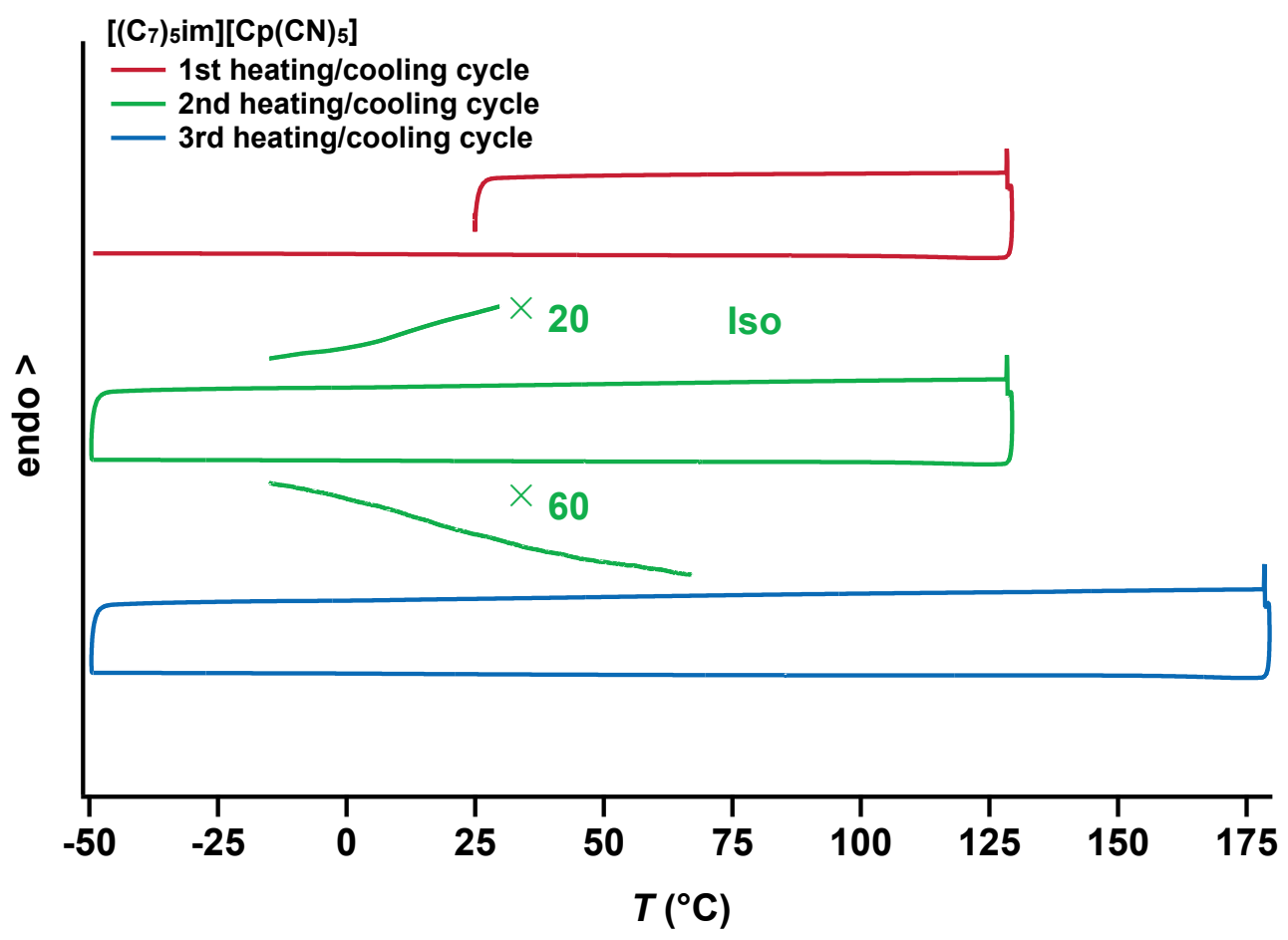

Figure S17. DSC data recorded for compound $5-\mathbf{C p}(\mathbf{C N})_{5^{-7}}$ at a heating rate of $10{ }^{\circ} \mathrm{C} \mathrm{min}^{-1}$ and a cooling rate of $5{ }^{\circ} \mathrm{C} \min ^{-1}$ under an atmosphere of $\mathrm{N}_{2}$. Endothermic peaks point upward.

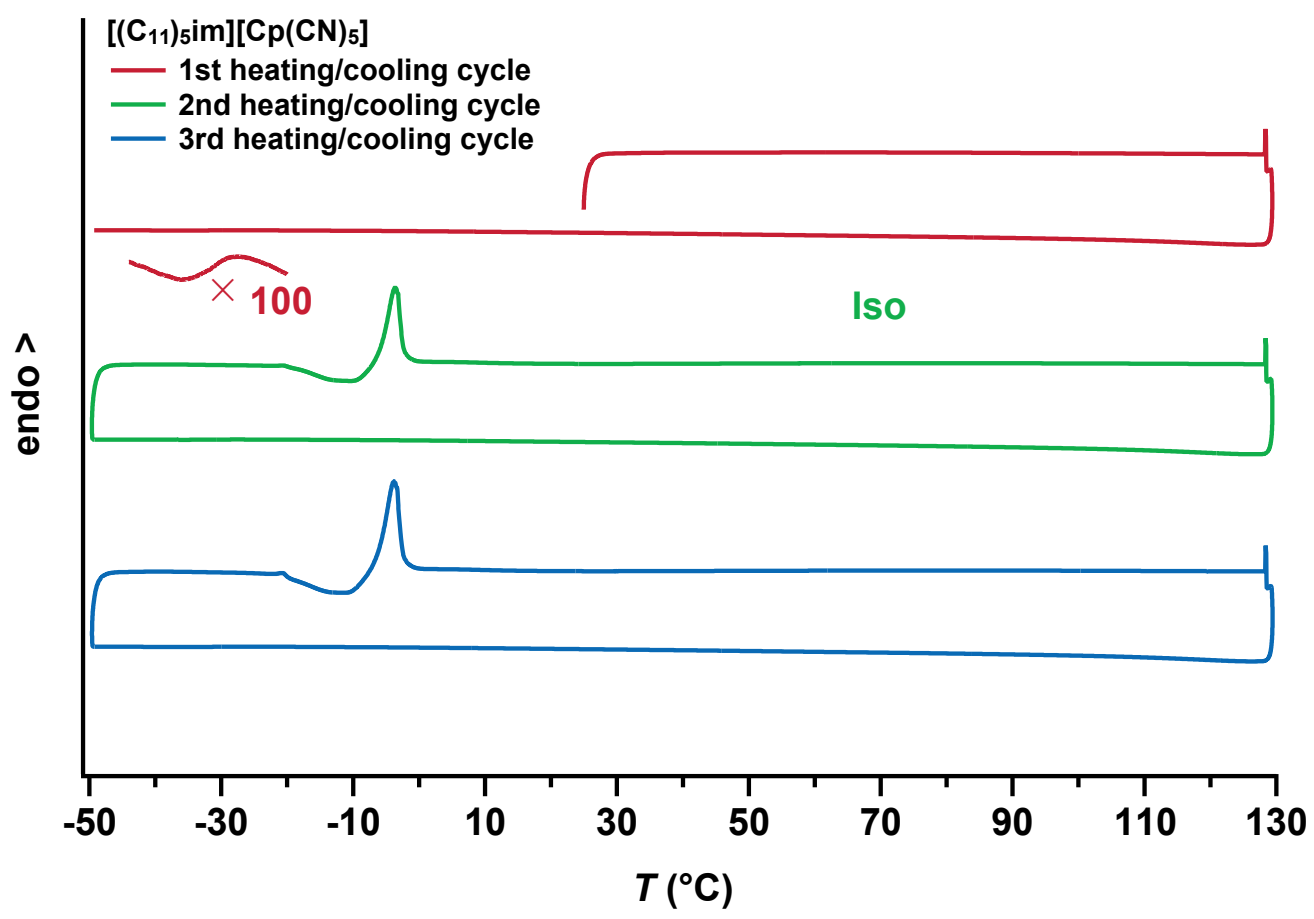

Figure S18. DSC data recorded for compound $5-\mathbf{C p}(\mathbf{C N})_{5}-11$ at a heating rate of $10{ }^{\circ} \mathrm{C} \mathrm{min}^{-1}$ and a cooling rate of $5{ }^{\circ} \mathrm{C} \mathrm{min}^{-1}$ under an atmosphere of $\mathrm{N}_{2}$. Endothermic peaks point upward. 


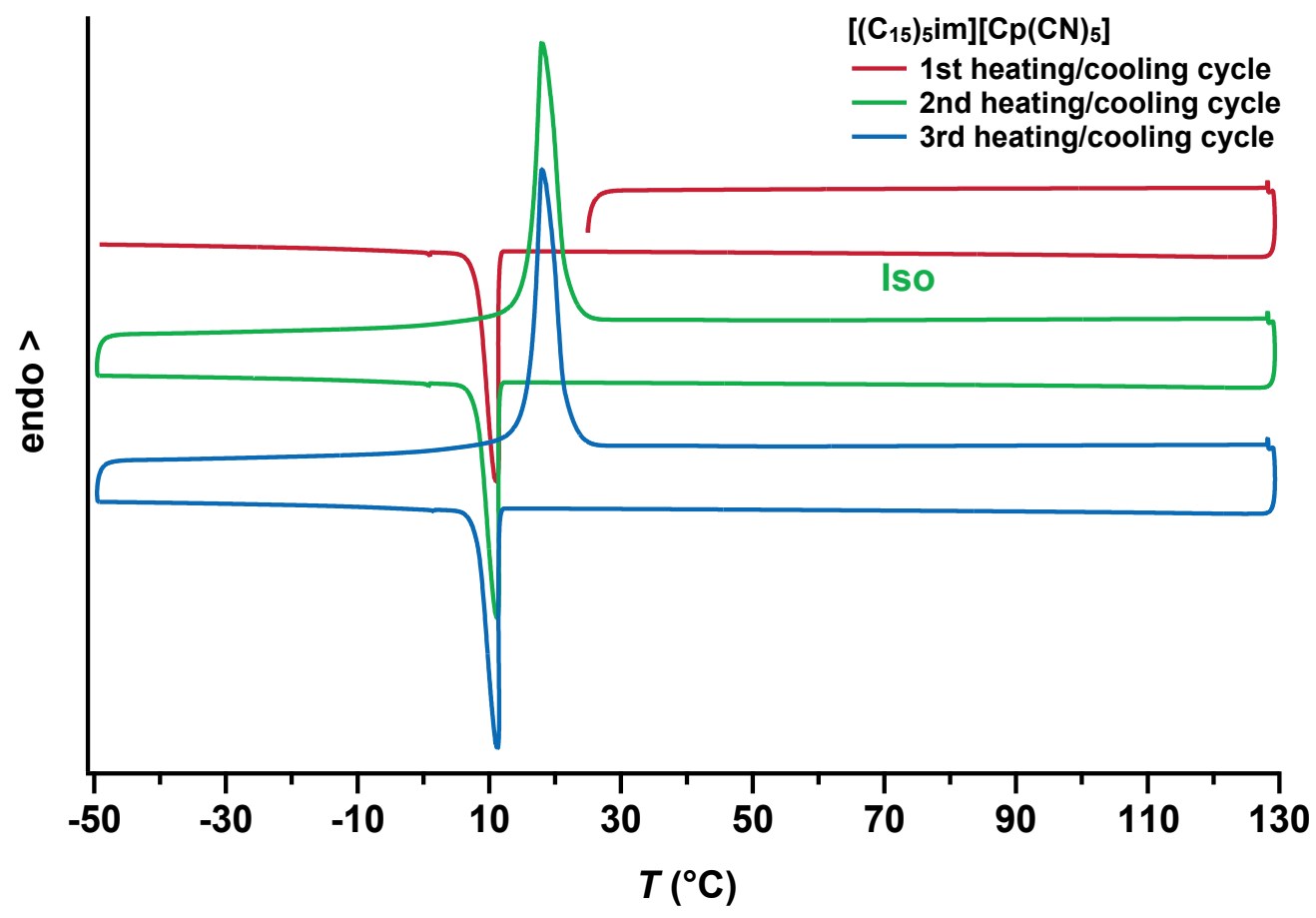

Figure S19. DSC data recorded for compound $5-\mathbf{C p}(\mathbf{C N})_{5}-15$ at a heating rate of $10{ }^{\circ} \mathrm{C} \mathrm{min}^{-1}$ and a cooling rate of $5{ }^{\circ} \mathrm{C} \min ^{-1}$ under an atmosphere of $\mathrm{N}_{2}$. Endothermic peaks point upward. 
(a)

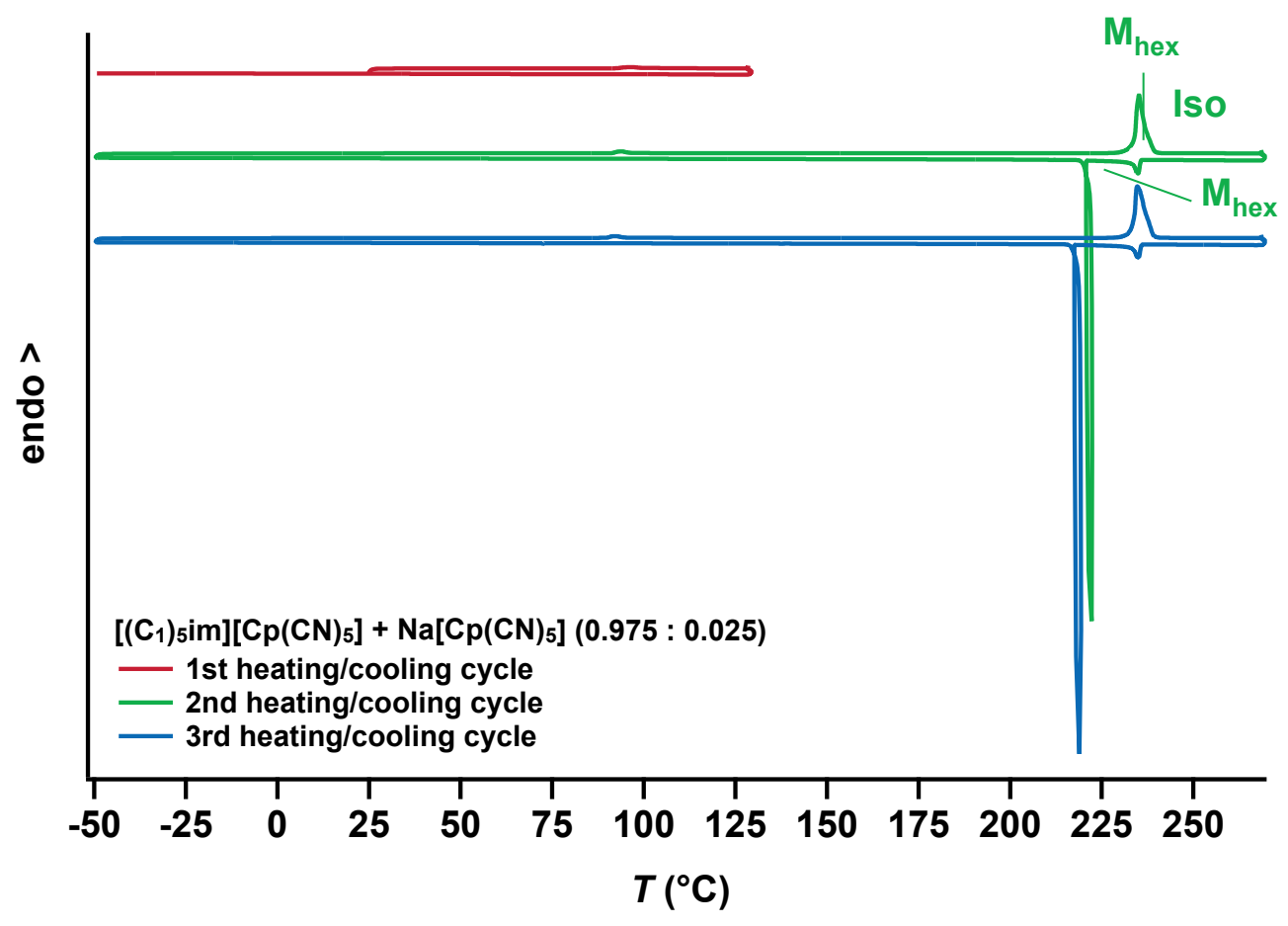

(b)

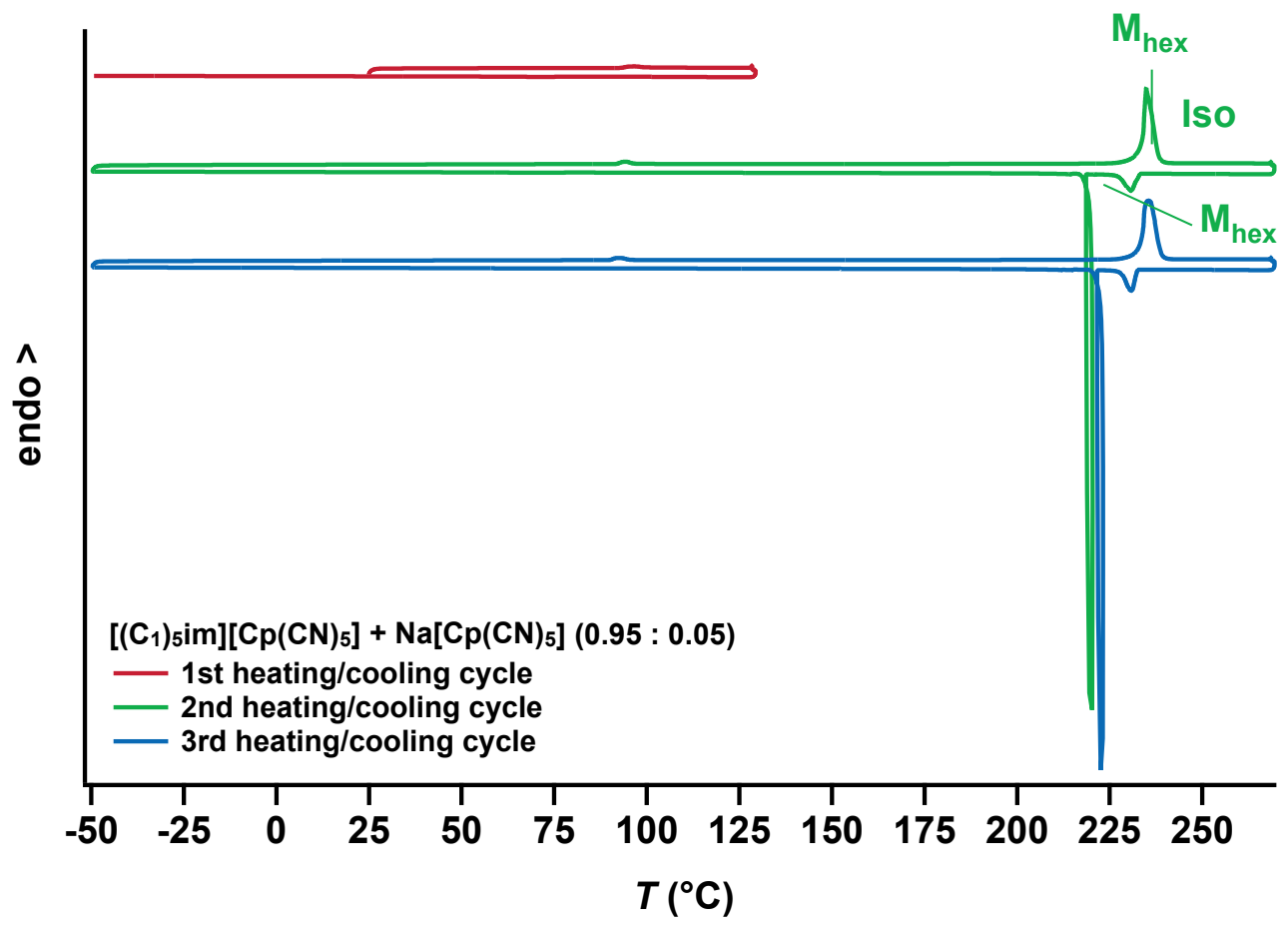

Figure S20. DSC data recorded for mixtures of $\mathbf{5 - C p}(\mathbf{C N})_{5}-\mathbf{1}$ and $\mathrm{Na}\left[\mathrm{Cp}(\mathrm{CN})_{5}\right]$ (molar ratios: (a) 0.975 : 0.025 and (b) $0.95: 0.05$ ) at a heating rate of $10{ }^{\circ} \mathrm{C} \mathrm{min}^{-1}$ and a cooling rate of $5{ }^{\circ} \mathrm{C} \min ^{-1}$ under an atmosphere of $\mathrm{N}_{2}$. Endothermic peaks point upward. 


\subsection{Table summarizing thermal data}

Table S2. Phase transition temperatures and thermal data recorded for the pentaalkylimidazolium salts 5-X-n. ${ }^{a}$

\begin{tabular}{|c|c|c|c|c|c|}
\hline Compound & Transition $^{b}$ & $T\left({ }^{\circ} \mathrm{C}\right)^{c}$ & $\Delta H\left(\mathrm{~kJ} \mathrm{~mol}^{-1}\right)^{d}$ & $\Delta S\left(\mathrm{~J} \mathrm{~K}^{-1} \mathrm{~mol}^{-1}\right)^{e}$ & $T_{1 \%}\left({ }^{\circ} \mathrm{C}\right)^{f}$ \\
\hline 5-I-1 & $\mathrm{Cr} \rightarrow \mathrm{Iso}^{g}$ & $214^{g}$ & $22.7^{g}$ & $\sim 47^{g}$ & $\sim 231$ \\
\hline 5-Br-7 & $\mathrm{S} \rightarrow \mathrm{Iso}^{h}$ & $46^{i}$ & 1.8 & $\sim 6$ & $\sim 188$ \\
\hline 5-Br-11 & $\mathrm{S} \rightarrow \mathrm{Iso}^{j}$ & $40,54,59^{j}$ & $42.0^{j}$ & ${ }^{j}$ & $\sim 183$ \\
\hline \multirow[t]{2}{*}{ 5-Br-15 } & $\mathrm{S}_{1} \rightarrow \mathrm{S}_{2}$ & 40 & 2.9 & 9 & $\sim 193$ \\
\hline & $\mathrm{S}_{2} \rightarrow \mathrm{Iso}^{k}$ & $78,81^{k}$ & $82.8^{k}$ & $-{ }^{k}$ & \\
\hline $5-\mathrm{NO}_{3}-1$ & $\mathrm{Cr} \rightarrow \mathrm{Iso}^{l}$ & $154^{l}$ & $13.2^{l}$ & $\sim 31^{l}$ & $\sim 267$ \\
\hline \multirow[t]{3}{*}{$5-\mathrm{NO}_{3}-7$} & $\mathrm{~S}_{1}+$ Iso $\rightarrow \mathrm{S}_{2}^{m}$ & $-8^{i}$ & -12.3 & $\sim-46$ & $\sim 175$ \\
\hline & $\mathrm{S}_{2} \rightarrow \mathrm{S}_{1}+\mathrm{Iso}^{m}$ & $19^{i}$ & 11.4 & $\sim 39$ & \\
\hline & $\mathrm{S}_{1}+\mathrm{Iso} \rightarrow \mathrm{Iso}^{m}$ & 45 & 1.1 & 3 & \\
\hline \multirow[t]{2}{*}{$5-\mathrm{NO}_{3}-11$} & $\mathrm{~S}_{1} \rightarrow \mathrm{S}_{2}$ & $5^{i}$ & 4.2 & $\sim 15$ & $\sim 185$ \\
\hline & $\mathrm{S}_{2} \rightarrow \mathrm{Iso}^{n}$ & $52^{n}$ & $33.1^{n}$ & $\sim 102^{n}$ & \\
\hline \multirow[t]{2}{*}{$5-\mathrm{NO}_{3}-15$} & $\mathrm{~S}_{1} \rightarrow \mathrm{S}_{2}^{o}$ & $41^{\circ}$ & $\sim 27.2^{\circ}$ & $\sim 87^{\circ}$ & $\sim 204$ \\
\hline & $\mathrm{S}_{2} \rightarrow$ Iso & $72^{i}$ & 77.6 & $\sim 225$ & \\
\hline \multirow[t]{3}{*}{$5-\mathrm{Cp}(\mathrm{CN})_{5}-1$} & $\mathrm{Cr}_{1} \rightarrow \mathrm{Cr}_{2}$ & 93 & 0.8 & 2 & $\sim 369$ \\
\hline & $\mathrm{Cr}_{2} \rightarrow \mathrm{M}_{\mathrm{hex}}$ & 234 & 16.4 & 32 & \\
\hline & $\mathrm{M}_{\text {hex }} \rightarrow$ Iso & 240 & 11.9 & 23 & \\
\hline 5-Cp(CN) $)_{5}-7$ & $\mathrm{~g} \rightarrow$ Iso & 14 & - & - & $\sim 192$ \\
\hline \multirow[t]{2}{*}{ 5-Cp(CN) $)_{5}^{-11}$} & $\mathrm{~g} \rightarrow \mathrm{S}^{p}$ & $-11^{i}$ & -7.7 & $\sim-29$ & $\sim 175$ \\
\hline & $\mathrm{S} \rightarrow \mathrm{Iso}^{p}$ & -6 & 9.7 & 36 & \\
\hline $5-\mathrm{Cp}(\mathrm{CN})_{5}-15$ & $\mathrm{~S} \rightarrow$ Iso & 16 & 92.9 & 321 & $\sim 221$ \\
\hline \multicolumn{6}{|c|}{$\begin{array}{l}{ }^{a} \text { This table contains additional data as compared with Table } 1 \text { in the main text. }{ }^{b} \text { Abbreviations: } \mathrm{Cr}, \mathrm{Cr}_{1}, \mathrm{Cr}_{2}= \\
\text { crystalline phase(s); } \mathrm{g}=\text { glass; } \mathrm{S}, \mathrm{S}_{1}, \mathrm{~S}_{2}=\text { solid phase(s) (not or only partially crystalline); } \mathrm{M}_{\text {hex }}=\text { plastic crystal } \\
\text { mesophase with } 2 \mathrm{D} \text { hexagonal symmetry; Iso = isotropic liquid phase. }{ }^{c} \text { Onset temperatures obtained by DSC } \\
\text { during the second heating run (unless indicated otherwise) at a heating rate of } 10{ }^{\circ} \mathrm{C} \mathrm{min}^{-1} \text { and under an } \\
\text { atmosphere of } \mathrm{N}_{2}\left(50 \mathrm{~mL} \mathrm{~min}^{-1} \text { ). A small hole was pierced into the lid of the DSC sample pans. The glass }\right. \\
\text { transition temperature was defined by the inflection point of the signal recorded in the respective DSC } \\
\text { thermogram. The first heating run was performed from } 25{ }^{\circ} \mathrm{C} \text { to } 130{ }^{\circ} \mathrm{C} \text {; the first cooling and subsequent cooling } \\
\text { runs were run to }-50{ }^{\circ} \mathrm{C} .{ }^{d} \text { Enthalpy change. }{ }^{e} \text { Entropy change. }{ }^{f} \text { Temperature at which } 1 \% \text { weight loss was }\end{array}$} \\
\hline
\end{tabular}


measured by TGA at a rate of $5{ }^{\circ} \mathrm{C} \mathrm{min}-1$ and under an atmosphere of $\mathrm{N}_{2}$ (neglecting the potential initial small weight loss attributed to the release of $\mathrm{H}_{2} \mathrm{O}$ ). ${ }^{g}$ This transition involved at least two, partially resolved transitions between $212{ }^{\circ} \mathrm{C}$ and $218{ }^{\circ} \mathrm{C}$ (see also Figure S8). The peak temperature of the most intense signal and the total enthalpy change are listed. Based on the relatively small estimated value for $\Delta S$ and visual inspection of the recorded DSC thermograms, a plastic crystal phase may occur over a narrow temperature range. ${ }^{h}$ The data in Table S2 were obtained from the third heating run (see also Figure S9). The pristine sample was obtained as a highly viscous oil or wax at ambient temperature, even after prolonged drying under reduced pressure. When examined by POM, the sample appeared faintly birefringent before it fully melted to an isotropic liquid at $\sim 46$ ${ }^{\circ} \mathrm{C}$. We surmise that the viscous state at ambient temperature represents an incompletely crystallized phase. ${ }^{i}$ Peak temperature. ${ }^{j}$ This transition involved three, partially resolved transitions between $30{ }^{\circ} \mathrm{C}$ and $65{ }^{\circ} \mathrm{C}($ see also Figure S10). The peak temperatures of the three signals and the total enthalpy change are listed. ${ }^{k}$ This transition involved two, partially resolved transitions between $66{ }^{\circ} \mathrm{C}$ and $86{ }^{\circ} \mathrm{C}$ (see also Figure S11). The peak temperatures of the two signals and the total enthalpy change are listed. ${ }^{l}$ This transition involved at least two, partially resolved transitions between $149^{\circ} \mathrm{C}$ and $159^{\circ} \mathrm{C}$ (see also Figure S12). The peak temperature of the most intense signal and the total enthalpy change are listed. Based on the relatively small estimated value for $\Delta S$ and visual inspection of the recorded DSC thermograms, a plastic crystal phase may occur over a narrow temperature range. ${ }^{m}$ The pristine sample was obtained as a viscous liquid-like substance. POM experiments revealed that the pristine sample also contained a fraction of small crystals, which melted between $\sim 33{ }^{\circ} \mathrm{C}$ and $\sim 45{ }^{\circ} \mathrm{C}$. Upon cooling, this crystal modification (i.e., " $\mathrm{S}_{1}$ ") formed again in a melt of non-solidified material (i.e., "Iso"); during the second heating run, recrystallization took place and resulted in a solid sample (i.e., " $\mathrm{S}_{2}$ ") which subsequently partially melted again (to form " $\mathrm{S}_{1}+$ Iso"). The data in Table S2 were obtained from the third heating run (see also Figure S13). ${ }^{n}$ This transition involved two, partially resolved transitions between $33{ }^{\circ} \mathrm{C}$ and $56{ }^{\circ} \mathrm{C}$ (see also Figure S14). The peak temperature of the most intense signal and the total enthalpy change are listed. ${ }^{o}$ This transition involved at least two, partially resolved transitions between $10{ }^{\circ} \mathrm{C}$ and $\sim 52{ }^{\circ} \mathrm{C}$ (see also Figure $\mathrm{S} 15$ ). The peak temperature of the most intense signal and the total enthalpy change are listed. ${ }^{p}$ The pristine sample was obtained as a liquid. Upon cooling, a glass transition was observed at ca. $-31{ }^{\circ} \mathrm{C}$. During the subsequent heating run, recrystallization took place. The data in Table S2 were obtained from the third heating run (see also Figure S18).

Similar enthalpy values were determined upon inspection of the melting and clearing endotherms in the DSC traces that were recorded for $\mathbf{5 - C p}(\mathbf{C N})_{5}-\mathbf{1}$ (Table S2). Likewise, similar observations were reported by Ros and co-workers for the mesomorphic, alkyl-free, indene- and pseudoazulene-based compounds. ${ }^{9,10}$ 


\section{Thermogravimetric analysis (TGA) data}

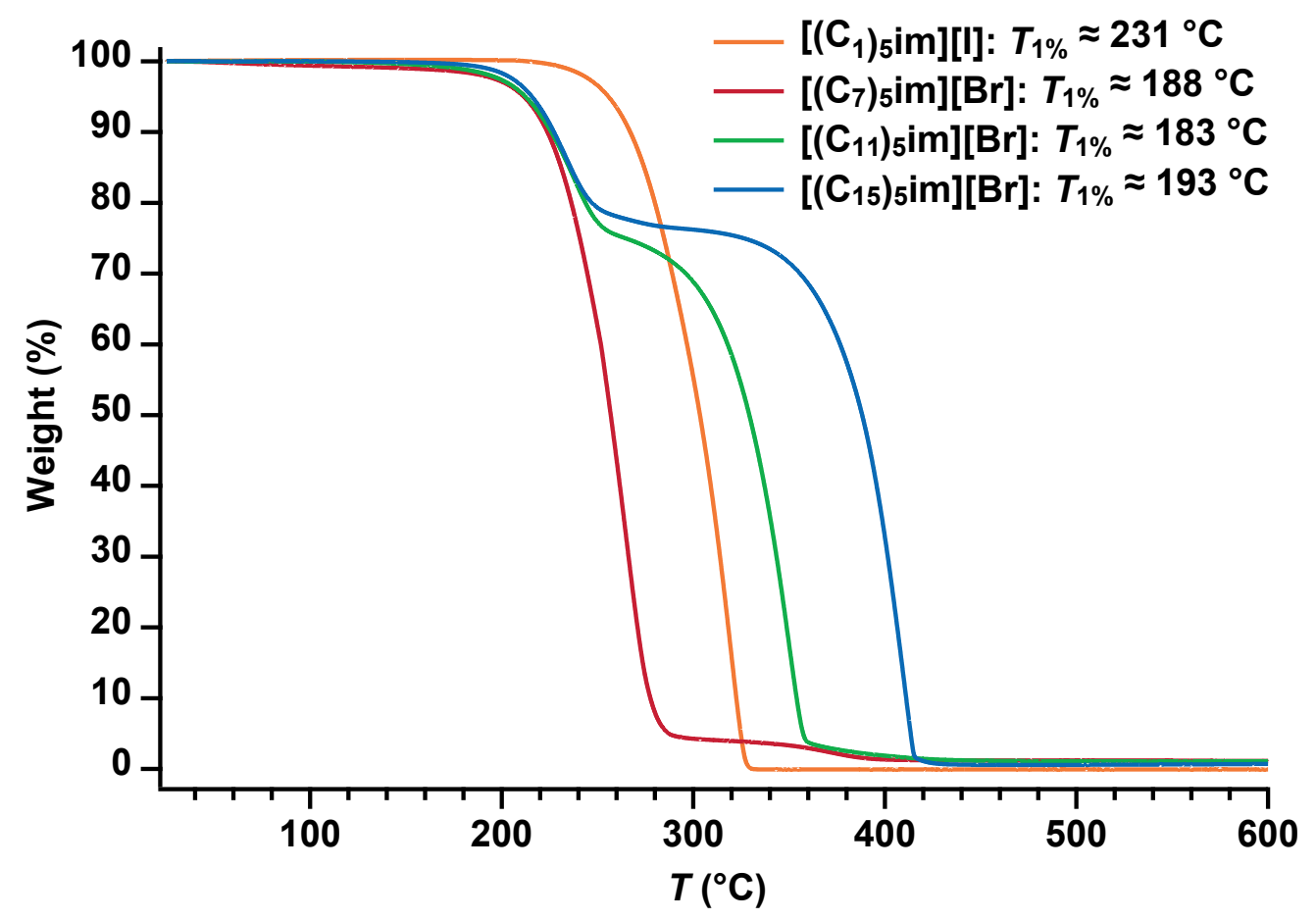

Figure S21. TGA data recorded for halide salts 5-I-1 and 5-Br- $\boldsymbol{n}(n=7,11,15)$ (heating rate of $5{ }^{\circ} \mathrm{C} \min ^{-1}$; $\mathrm{N}_{2}$ atmosphere). The $T_{1 \%}$ value represents the temperature at which $1 \%$ weight loss was measured (neglecting the initial small weight loss attributed to the release of $\mathrm{H}_{2} \mathrm{O}(<1 \%$ for 5-Br-7 $\left.)\right)$. The observed weight losses for 5-Br-11 and 5-Br-15 indicate that the first decomposition step involves the expulsion of $\mathrm{C}_{11} \mathrm{H}_{23} \mathrm{Br}$ and $\mathrm{C}_{15} \mathrm{H}_{31} \mathrm{Br}$, respectively. 


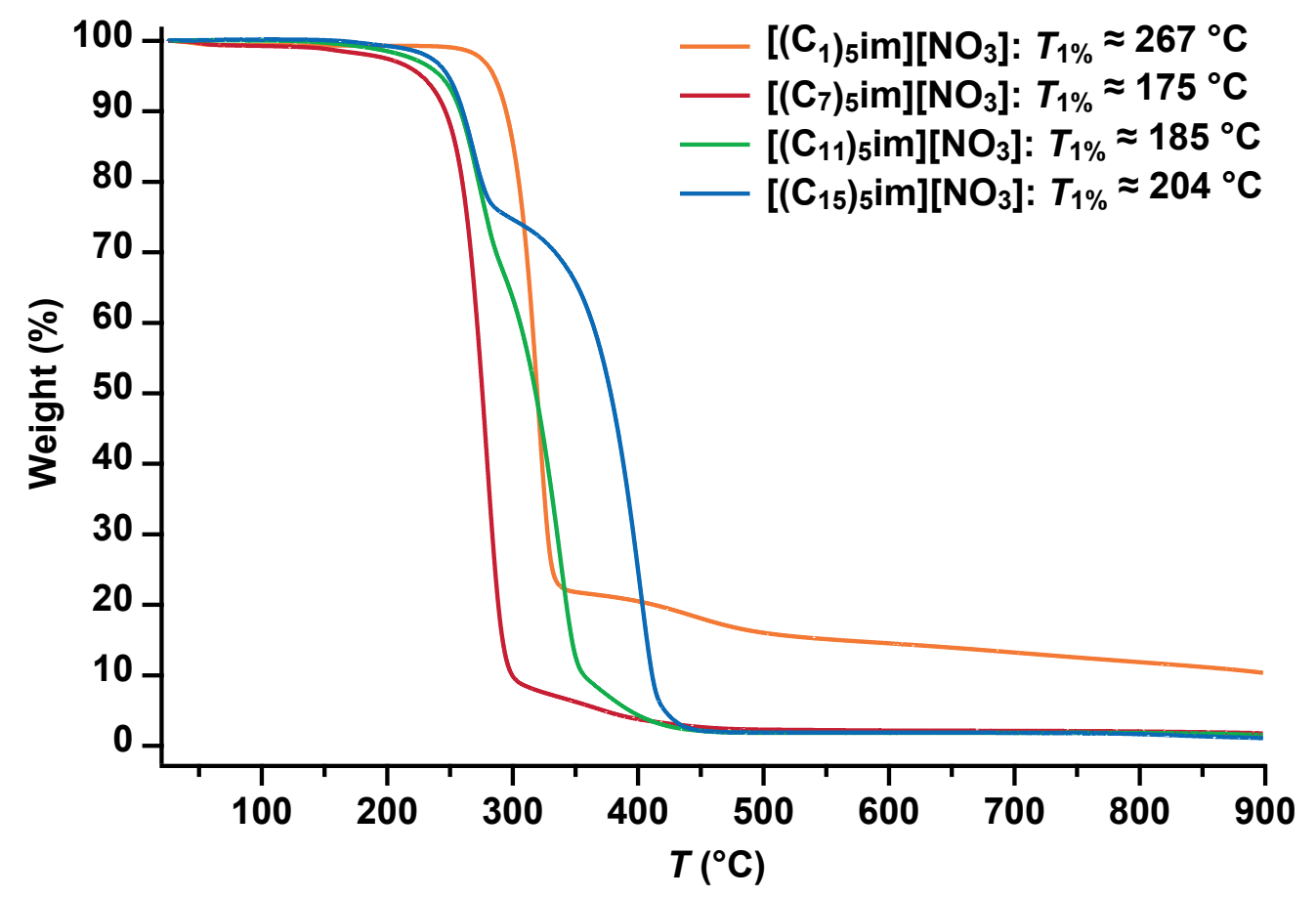

Figure S22. TGA data recorded for nitrate salts $\mathbf{5}-\mathrm{NO}_{3}-\boldsymbol{n}(n=1,7,11,15)$ (heating rate of $5{ }^{\circ} \mathrm{C} \min ^{-1} ; \mathrm{N}_{2}$ atmosphere). The $T_{1 \%}$ value represents the temperature at which $1 \%$ weight loss was measured (neglecting the initial small weight loss attributed to the release of $\mathrm{H}_{2} \mathrm{O}\left(\sim 0.6 \%\right.$ for $\mathbf{5}-\mathrm{NO}_{3}-\mathbf{1}$ and $\sim 0.8 \%$ for $\left.\left.\mathbf{5}-\mathrm{NO}_{3}-\mathbf{7}\right)\right)$. 


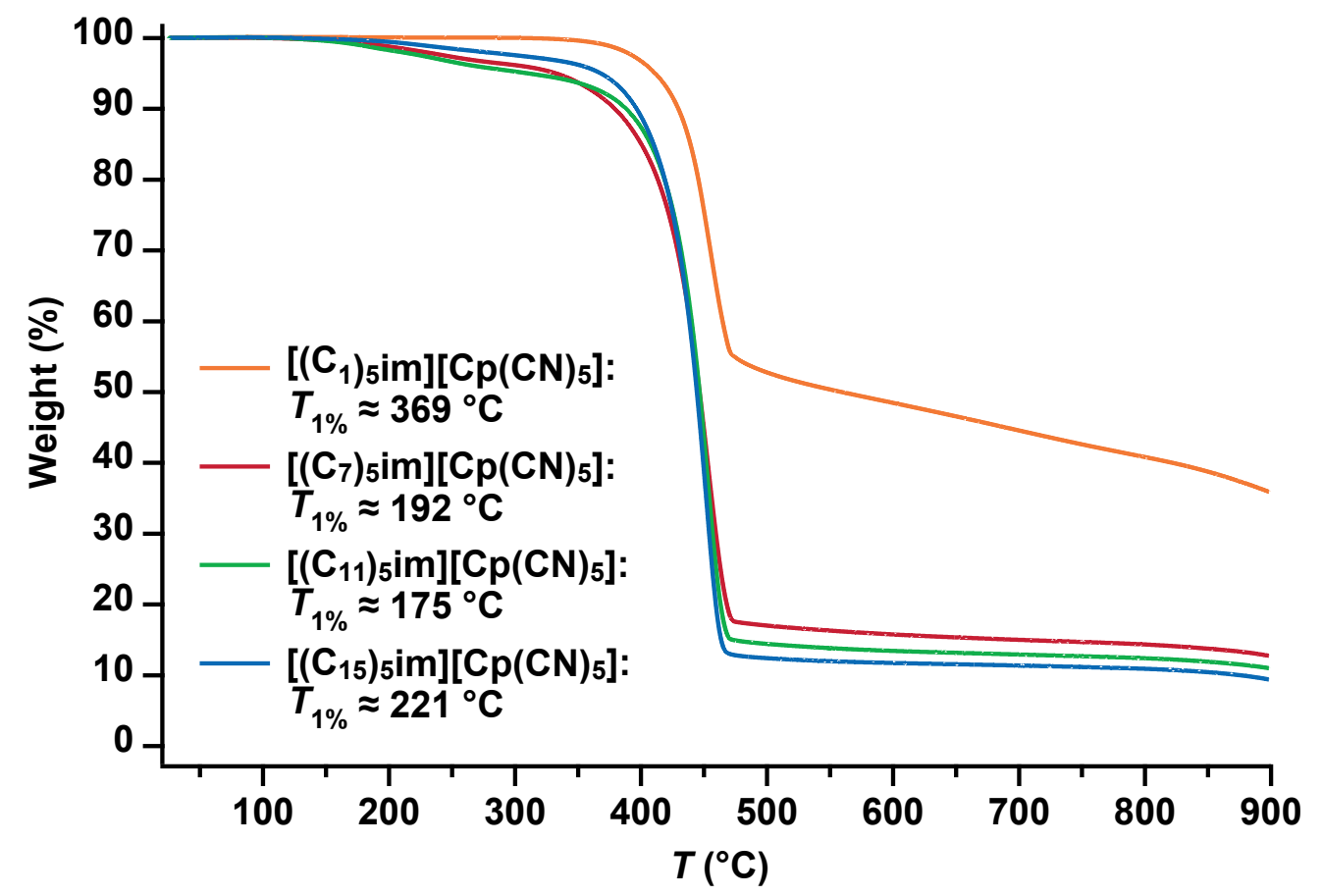

Figure S23. TGA data recorded for pentacyanocyclopentadienide salts $\mathbf{5 - C} \mathbf{p}(\mathbf{C N})_{5}-\boldsymbol{n}(n=1,7,11,15)$ (heating rate of $5{ }^{\circ} \mathrm{C} \mathrm{min}^{-1} ; \mathrm{N}_{2}$ atmosphere). The $T_{1 \%}$ value represents the temperature at which $1 \%$ weight loss was measured. 


\section{Simultaneous TGA-DSC data}

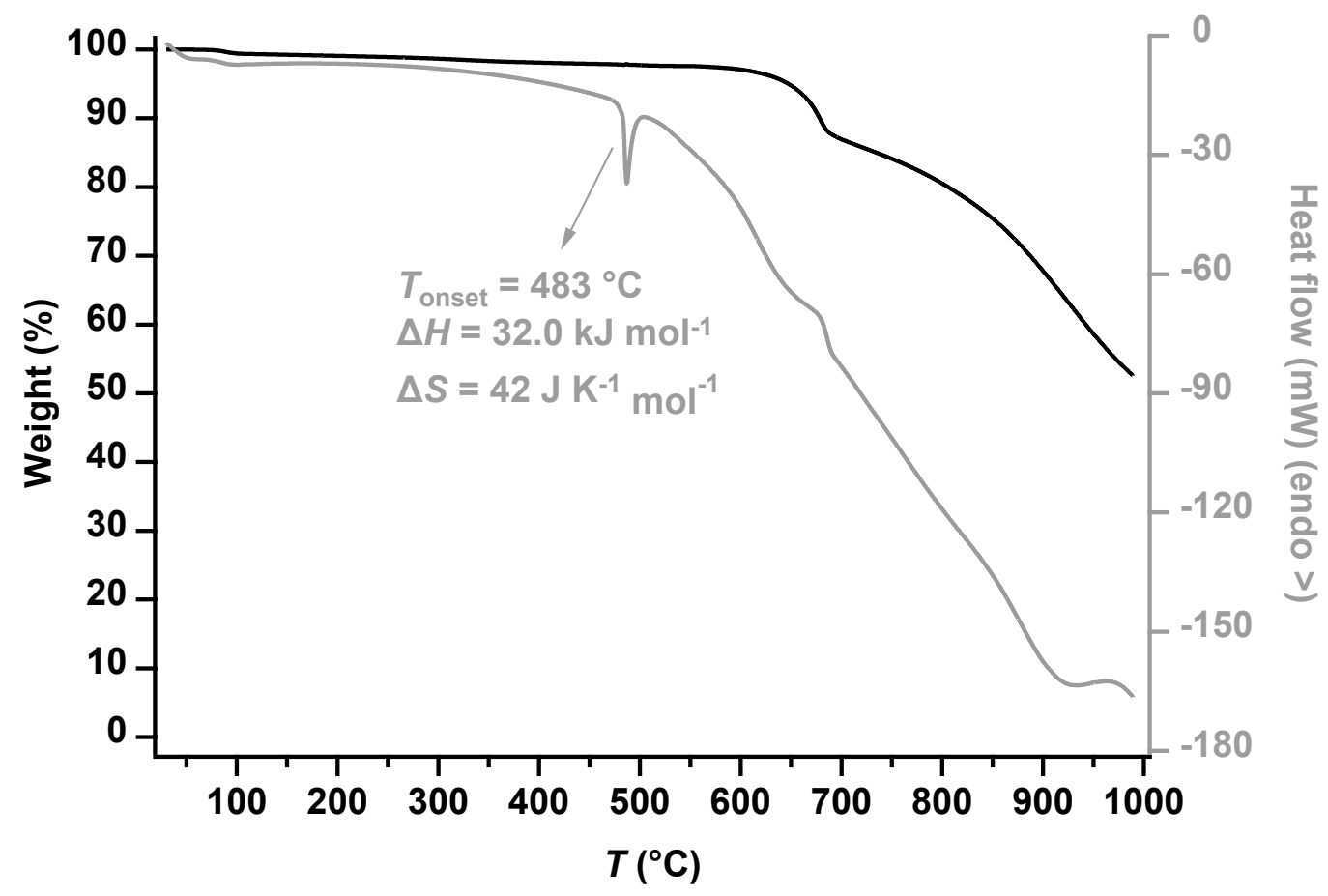

Figure S24. Simultaneous TGA-DSC data recorded for sodium pentacyanocyclopentadienide, $\mathrm{Na}\left[\mathrm{Cp}(\mathrm{CN})_{5}\right]$ (heating rate of $10{ }^{\circ} \mathrm{C}^{-1}{ }^{-1} ; \mathrm{N}_{2}$ atmosphere). In the DSC thermogram, depicted in grey, endothermic peaks point downward. 


\section{Single-crystal X-ray diffraction data}

We were unable to grow X-ray quality crystals for compounds $\mathbf{5}-\mathbf{X}-\mathbf{n}\left(\mathrm{X}^{-}=\mathrm{Br}^{-},\left[\mathrm{NO}_{3}\right]^{-} ; n=7,11\right.$, 15) and 5- $\mathrm{NO}_{3}-\mathbf{1}$.

\subsection{Compound 5-I-1 ${ }^{11}$}

Colorless, clear, transparent, block-shaped single crystals of 5-I-1 were obtained by slow evaporation of a dichloromethane solution.

X-ray intensity data were collected at $123 \mathrm{~K}$ on a Rigaku XtaLAB P200 diffractometer equipped with a Pilatus $200 \mathrm{~K}$ detector, using $\omega$ scans and $\mathrm{Cu} \mathrm{K} \alpha$ radiation (wavelength $\lambda=1.54187 \AA$ ). The images were interpreted and integrated with the program CrystalClear-SM Expert (version $2.1 \mathrm{~b} 45,2015)$ from Rigaku. ${ }^{12}$ After indexation of the X-ray diffraction pattern, the unit cell was refined based on 816 reflections (i.e. $4 \%$ of the total of 20114 reflections that were measured) and data reduction, scaling and numerical absorption corrections (using the ABSCOR program ${ }^{13}$ ) were performed. The final completeness was $99.6 \%$ to $\theta_{\max }=68.304^{\circ}$. Using Olex $2,{ }^{14}$ the structure was solved with the ShelXS ${ }^{15}$ structure solution program using Direct Methods and refined with the ShelXL ${ }^{16}$ refinement package using full-matrix least-squares minimization on $F^{2}$. Non-hydrogen atoms were refined anisotropically. Hydrogen atom positions were calculated geometrically and refined in riding mode. "Short contacts" were identified with the Platon software package. ${ }^{17,18}$ CCDC-1871620 contains the supplementary crystallographic data for this compound. These data can be obtained free of charge from The Cambridge Crystallographic Data Centre via www.ccdc.cam.ac.uk/structures/.

Crystallographic data for 5-I-1: $\mathrm{C}_{18} \mathrm{H}_{15} \mathrm{IN}_{2} ; M=266.13 \mathrm{~g} \mathrm{~mol}^{-1}$; orthorhombic; space group Pcab (No. 61); $a=11.131(4) \AA, b=12.188(4), c=15.203(6) \AA ; \alpha=\beta=\gamma=90^{\circ} ; V=$ 2062.5(13) $\AA^{3} ; T=123 \mathrm{~K} ; Z=8 ; Z^{\prime}=1 ; \rho_{\text {calcd. }}=1.714 \mathrm{~g} \mathrm{~cm}^{-3} ; \mu(\mathrm{Cu} \mathrm{K \alpha})=23.961 \mathrm{~mm}^{-1} ; F(000)$ $=1040$; crystal size $0.20 \times 0.15 \times 0.13 \mathrm{~mm}^{3} ; 20114$ reflections measured $\left(7.268^{\circ} \leq \theta \leq 68.304^{\circ}\right)$, 1866 unique reflections $\left(R_{\text {int }}=0.0550\right)$ which were used in all calculations. The final $R_{1}$ was $0.0242(I>2 \sigma(I))$ and $w R_{2}$ was 0.0566 (all data). 


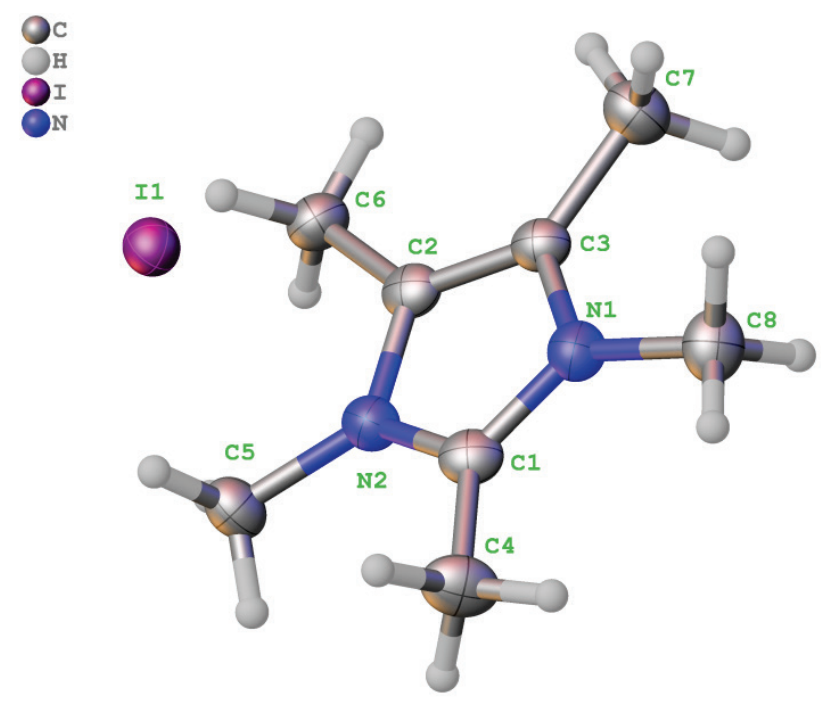

Figure S25. Asymmetric unit in the crystal structure of compound 5-I-1 with an atom labelling scheme. The thermal displacement ellipsoids were drawn at the $50 \%$ probability level.

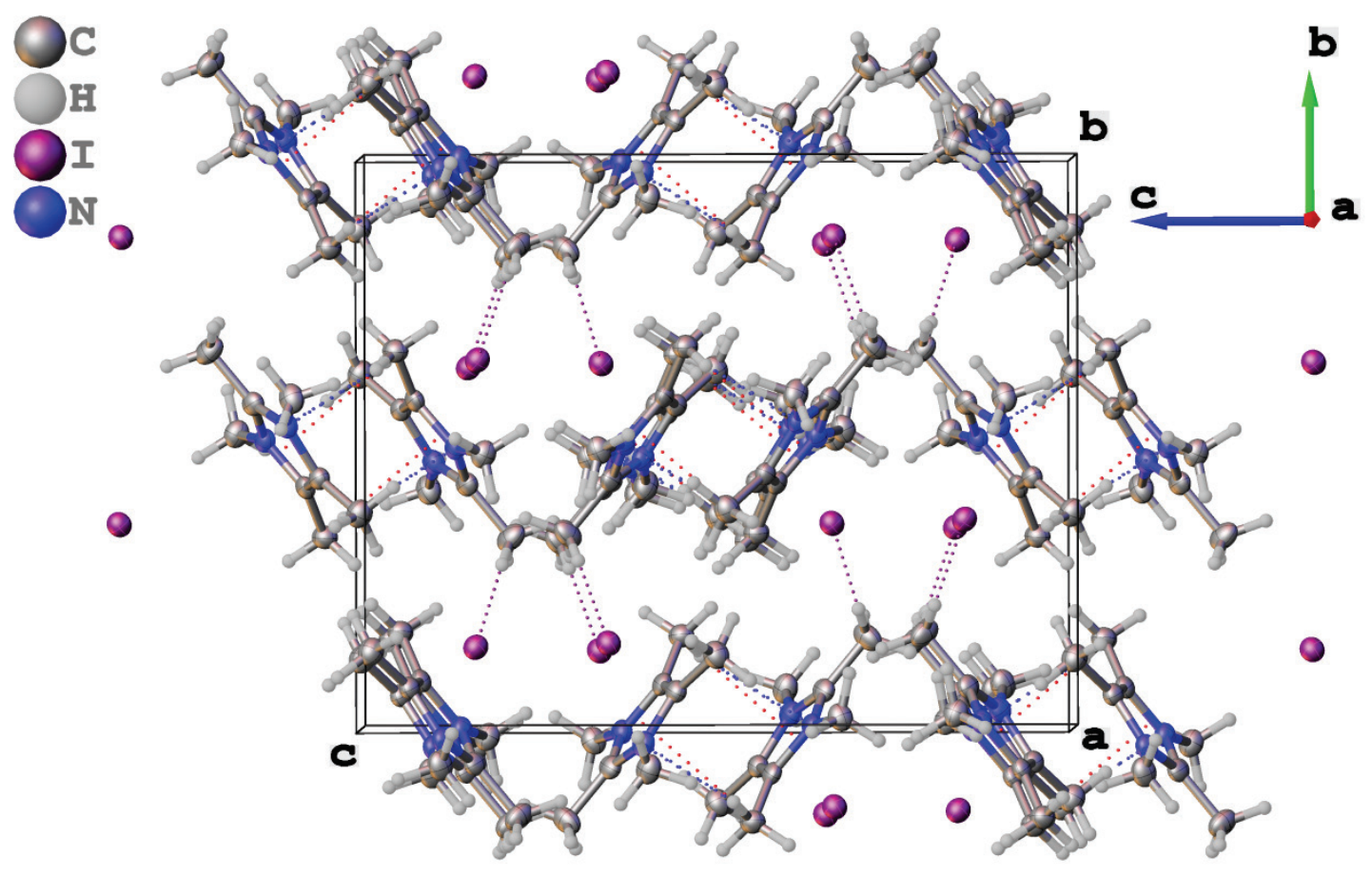

Figure S26. Packing in the crystal structure of compound 5-I-1, showing the linkage of the molecules through non-classical C-H $\cdots \mathrm{N}$ (blue dotted lines; see also Figure S27 and Table S3) and $\mathrm{C}-\mathrm{H} \cdots \mathrm{I}$ (purple dotted lines; see also Table S3) hydrogen bonds as well as hydrogen-to-ring ("C $\mathrm{C}-\mathrm{H} \cdots \pi$ ”) interactions (red dotted lines; see also Figure S27 and Table S4). Note the absence of columnar stacking of the imidazolium rings. 


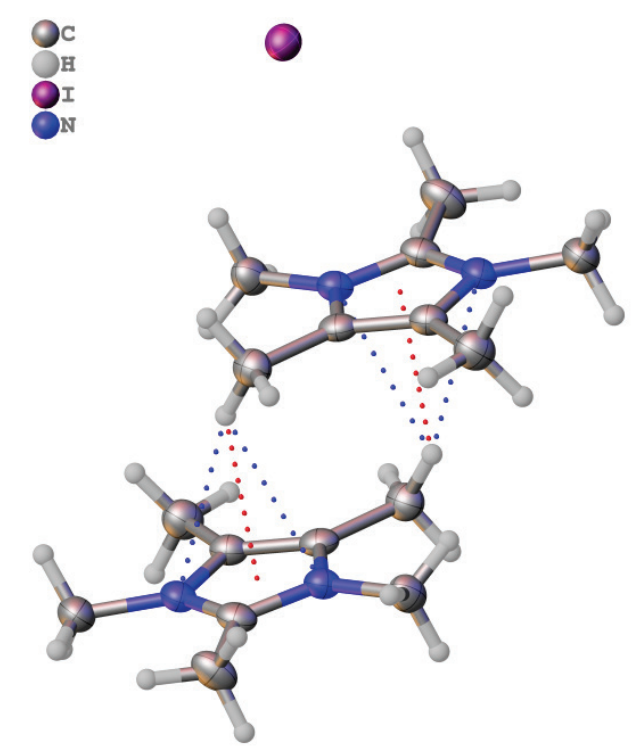

0

Figure S27. Detailed view of the packing in the crystal structure of compound 5-I-1, showing the linkage of the molecules through non-classical $\mathrm{C}-\mathrm{H} \cdots \mathrm{N}$ hydrogen bonds (blue dotted lines) as well as hydrogen-to-ring (" $\mathrm{C}-\mathrm{H} \cdots \pi$ ”) interactions (red dotted lines). 
Table S3. Geometry data (distances and angles) for the non-classical hydrogen bonds that were detected in the crystal structure of compound 5-I-1. ${ }^{a}$

\begin{tabular}{|c|c|c|c|c|}
\hline D-H $\cdots A$ & D-H $(\AA ̊)$ & $\mathbf{H} \cdots \mathbf{A}(\AA)$ & $\mathbf{D} \cdots \mathbf{A}(\AA)$ & D-H $\cdots$ A $\left({ }^{\circ}\right)$ \\
\hline C6-H6A $\cdots N 2^{i}$ & 0.96 & 2.82 & $3.433(4)$ & 122.7 \\
\hline C6-H6 $\cdots N 1^{i}$ & 0.96 & 2.98 & $3.910(4)$ & 164.5 \\
\hline C4-H4C $\cdots I^{i i}$ & 0.96 & 3.02 & $3.976(4)$ & 178 \\
\hline
\end{tabular}

Definition of the five-membered ring in the asymmetric unit $(C g$ indicates the centroid of the ring):

$C g 1=\mathrm{N} 1-\mathrm{C} 1-\mathrm{N} 2-\mathrm{C} 2-\mathrm{C} 3$

Table S4. Geometry data (distances and angles) for the hydrogen-to-ring ("C $-\mathrm{H} \cdots \pi$ ") interactions that were detected in the crystal structure of compound 5-I-1. ${ }^{a}$

\begin{tabular}{|cccc|}
\hline $\mathbf{C}-\mathbf{H} \cdots \boldsymbol{C g}$ & $\mathbf{H} \cdots \boldsymbol{C g}($ centroid $)(\AA)$ & $\mathbf{C} \cdots \boldsymbol{C g}($ centroid $)(\AA)$ & $\begin{array}{c}\mathbf{C}-\mathbf{H} \cdots \boldsymbol{C g}(\text { centroid }) \\
\left({ }^{\circ}\right)\end{array}$ \\
\hline $\mathbf{C 6}-\mathbf{H} 6 \mathrm{~A} \cdots \boldsymbol{C g} \mathbf{1}^{\mathbf{i}}$ & 2.69 & $3.530(3)$ & 146 \\
\hline${ }^{a}$ Symmetry code: $(\mathrm{i})-x, 1-y, 1 / 2-z$. & & \\
\hline
\end{tabular}




\subsection{Compound $5-\mathrm{Cp}(\mathrm{CN})_{5}-1$}

White, block-shaped single crystals of $\mathbf{5 - C p}(\mathbf{C N})_{5}-1$ were obtained by slow evaporation of a dichloromethane solution.

X-ray intensity data were collected at $123 \mathrm{~K}$ on a Rigaku XtaLAB P200 diffractometer equipped with a Pilatus $200 \mathrm{~K}$ detector, using $\omega$ scans and $\mathrm{Cu} \mathrm{K} \alpha$ radiation (wavelength $\lambda=1.54187 \AA$ ). The images were interpreted and integrated with the program CrystalClear-SM Expert (version $2.1 \mathrm{~b} 45,2015)$ from Rigaku. ${ }^{12}$ After indexation of the X-ray diffraction pattern, the unit cell was refined based on 4354 reflections (i.e. $11 \%$ of the total of 38401 reflections that were measured) and data reduction, scaling and numerical absorption corrections (using the ABSCOR program ${ }^{13}$ ) were performed. The final completeness was $99.3 \%$ to $\theta_{\max }=68.248^{\circ}$. Using Olex $2,{ }^{14}$ the structure was solved with the Olex2.solve ${ }^{19}$ structure solution program using charge flipping and refined with the ShelXL ${ }^{16}$ refinement package using full-matrix least-squares minimization on $F^{2}$. Non-hydrogen atoms were refined anisotropically. Hydrogen atom positions were calculated geometrically and refined in riding mode. The atomic coordinates and anisotropic displacement parameters of atoms N11-C21-N12-C26 were constrained using the "EXYZ" and "EADP" commands. "Short contacts" were identified with the Platon software package. ${ }^{17,18}$ CCDC1868291 contains the supplementary crystallographic data for this compound. These data can be obtained free of charge from The Cambridge Crystallographic Data Centre via www.ccdc.cam.ac.uk/structures/.

Crystallographic data for 5-Cp(CN) $)_{5}-1: \mathrm{C}_{18} \mathrm{H}_{15} \mathrm{~N}_{7} ; M=329.37 \mathrm{~g} \mathrm{~mol}^{-1}$; orthorhombic; space group Pcab (No. 61); $a=15.3281(18) \AA, b=21.355(2) \AA, c=21.815(3) \AA ; \alpha=\beta=\gamma=90^{\circ} ; V=$ $7140.6(15) \AA^{3} ; T=123 \mathrm{~K} ; Z=16 ; Z^{\prime}=2 ; \rho_{\text {calcd. }}=1.226 \mathrm{~g} \mathrm{~cm}^{-3} ; \mu(\mathrm{Cu} \mathrm{K \alpha})=0.636 \mathrm{~mm}^{-1} ; F(000)$ $=2752$; crystal size $0.30 \times 0.20 \times 0.17 \mathrm{~mm}^{3} ; 38401$ reflections measured $\left(7.052^{\circ} \leq \theta \leq 68.248^{\circ}\right)$, 6500 unique reflections $\left(R_{\text {int }}=0.0281\right)$ which were used in all calculations. The final $R_{1}$ was $0.0359(I>2 \sigma(I))$ and $w R_{2}$ was 0.1085 (all data). 

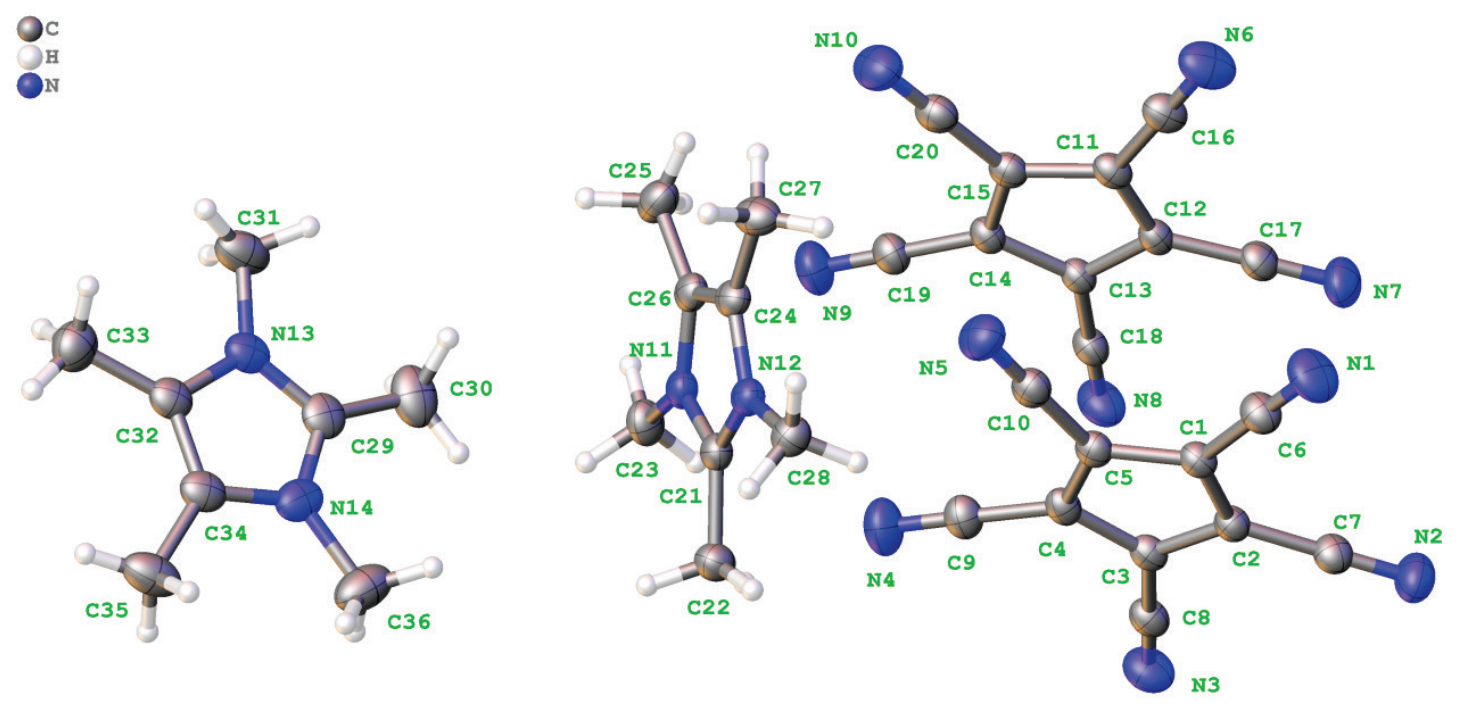

Figure S28. Asymmetric unit in the crystal structure of compound $5-\mathbf{C p}(\mathbf{C N})_{5}-\mathbf{1}$ with an atom labelling scheme. The thermal displacement ellipsoids were drawn at the $50 \%$ probability level. 


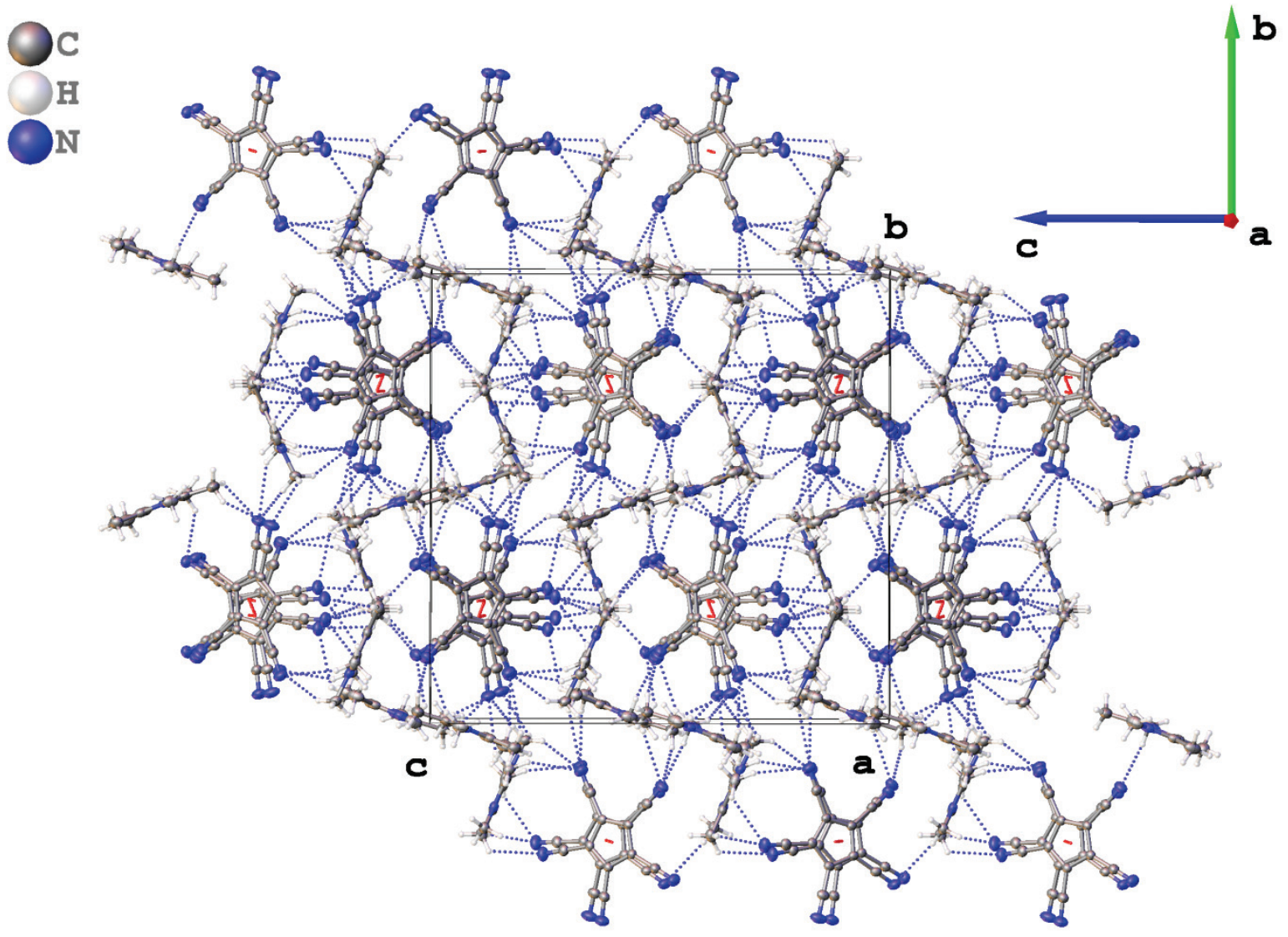

Figure S29. Packing in the crystal structure of compound $5-\mathbf{C p}(\mathbf{C N})_{5}-\mathbf{1}$, showing the columnar stacking of the $\left[\mathrm{Cp}(\mathrm{CN})_{5}\right]^{-}$anions in the direction of the $a$-axis (red dotted lines indicate the ring-to-ring interactions; see also Figure S30 and Table S6) and the linkage of the molecules through non-classical $\mathrm{C}-\mathrm{H} \cdots \mathrm{N}$ hydrogen bonds (blue dotted lines; see also Table S5). The columnar stacks of $\left[\mathrm{Cp}(\mathrm{CN})_{5}\right]^{-}$anions occupy the nodes of a hexagonal sublattice. 


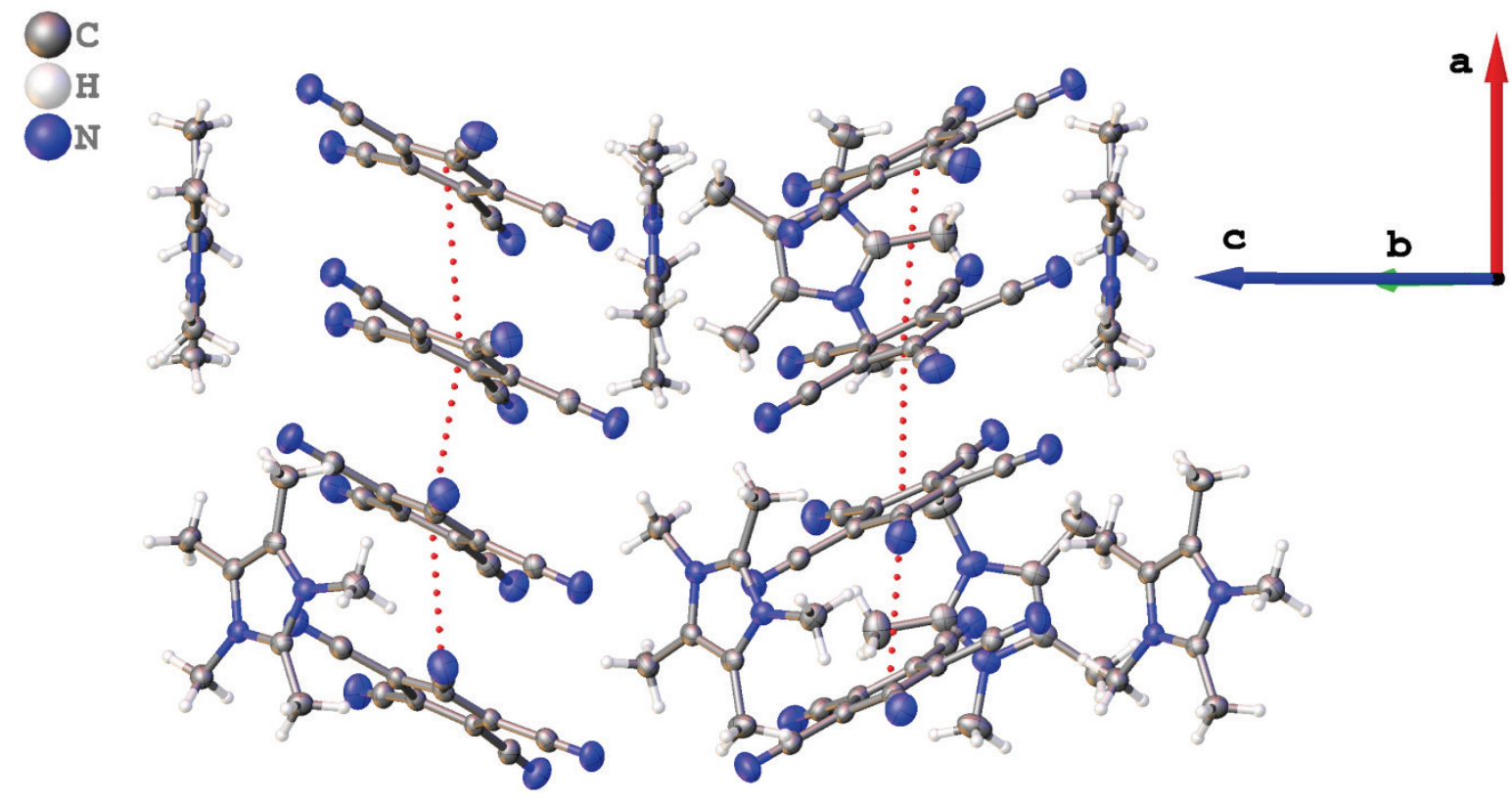

Figure S30. Packing in the crystal structure of compound $5-\mathbf{C p}(\mathbf{C N})_{5}-\mathbf{1}$, showing the ring-to-ring interactions between $\left[\mathrm{Cp}(\mathrm{CN})_{5}\right]^{-}$anions (red dotted lines). 

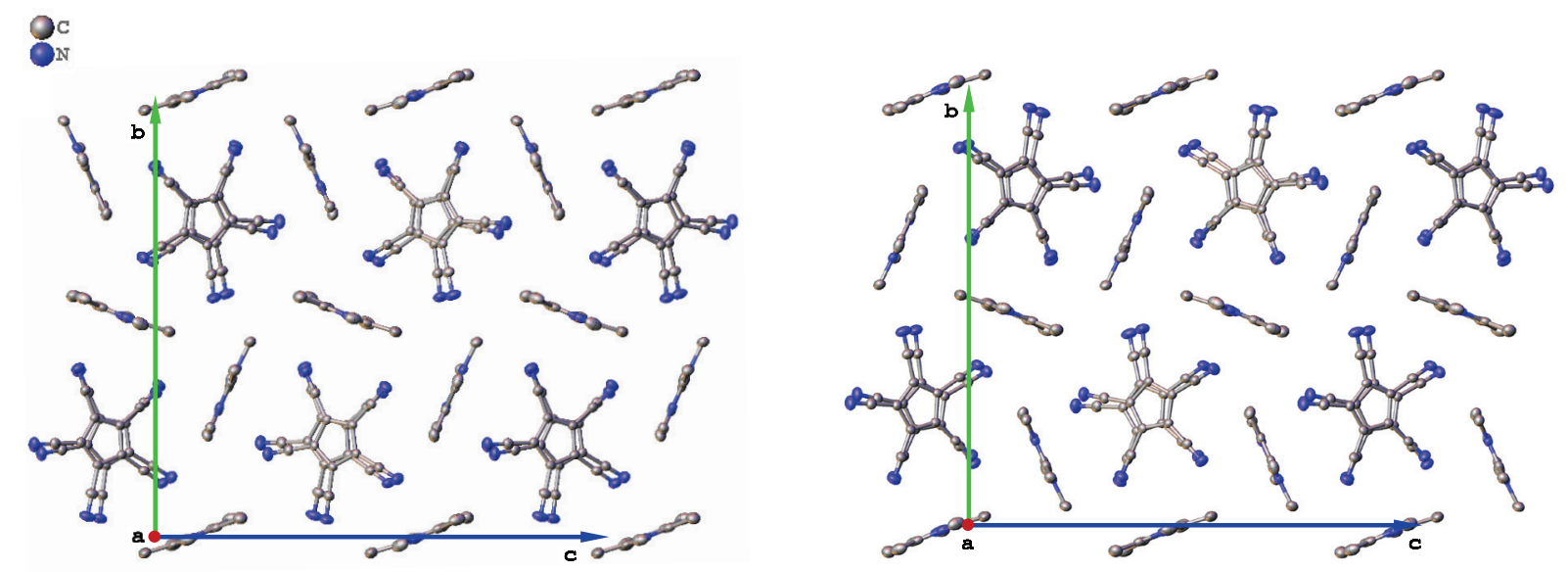

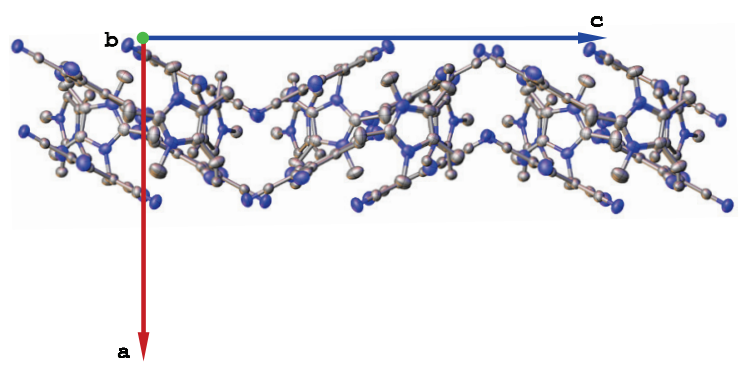

$x=0.1-0.3$

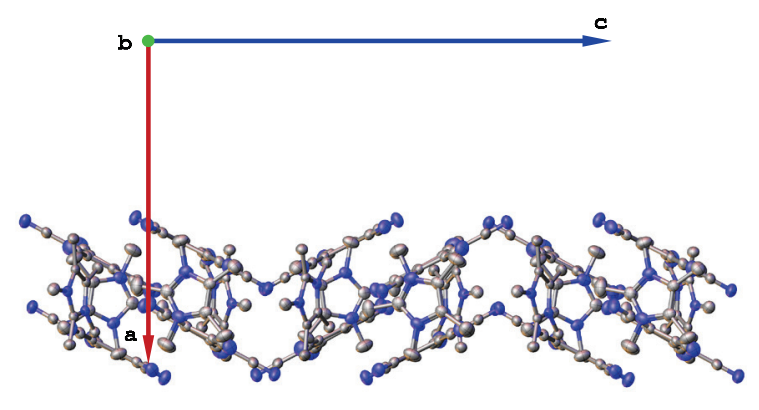

$x=0.6-0.9$

Figure S31. Packing in the crystal structure of compound $5-\mathbf{C p}(\mathbf{C N})_{5}-1$. The ions are arranged into layers defined by segments of two stacked $\left[\mathrm{Cp}(\mathrm{CN})_{5}\right]^{-}$anions that are tilted out of the $b \times c$ plane by $27.5^{\circ}$. The anions are surrounded by $\left[\left(\mathrm{C}_{1}\right)_{5} \mathrm{im}\right]^{+}$cations whose planes are orthogonal to the layers. The superposition of the layers yields columns of stacked anions, with intercalated cations providing optimal space-filling. 
Table S5. Geometry data (distances and angles) for the non-classical hydrogen bonds that were detected in the crystal structure of compound $\mathbf{5 - C p ( C N )})_{5}-{ }^{a}$

\begin{tabular}{|c|c|c|c|c|}
\hline D-H $\cdots A$ & D-H (Å) & $\mathbf{H} \cdots \mathbf{A}(\AA)$ & $\mathbf{D} \cdots \mathbf{A}(\AA ̊)$ & D-H $\cdots$ A $\left(^{\circ}\right)$ \\
\hline C23-H23A $\cdots \mathrm{N}^{\mathrm{i}}$ & 0.96 & 2.48 & $3.3796(17)$ & 157 \\
\hline $\mathrm{C} 28-\mathrm{H} 28 \mathrm{D} \cdots \mathrm{N} 2^{\mathrm{ii}}$ & 0.96 & 2.61 & $3.5631(17)$ & 170 \\
\hline C30-H30A $\cdots N 8^{\mathrm{iii}}$ & 0.96 & 2.62 & $3.1154(18)$ & 114 \\
\hline C35-H35A $\cdots N 9^{\mathrm{iii}}$ & 0.96 & 2.62 & $3.4201(17)$ & 141 \\
\hline C31-H31C $\cdots N^{\text {iv }}$ & 0.96 & 2.62 & $3.4603(19)$ & 146 \\
\hline
\end{tabular}

Definitions of the four five-membered rings in the asymmetric unit $(C g$ indicates the centroid of each ring):

$$
\begin{aligned}
& C g 1=\mathrm{C} 1-\mathrm{C} 2-\mathrm{C} 3-\mathrm{C} 4-\mathrm{C} 5 \\
& C g 2=\mathrm{C} 11-\mathrm{C} 12-\mathrm{C} 13-\mathrm{C} 14-\mathrm{C} 15 \\
& C g 3=\mathrm{N} 11-\mathrm{C} 21-\mathrm{N} 12-\mathrm{C} 26-\mathrm{C} 24 \\
& C g 4=\mathrm{N} 13-\mathrm{C} 29-\mathrm{N} 14-\mathrm{C} 34-\mathrm{C} 32
\end{aligned}
$$

Table S6. Geometry data for the ring-to-ring interactions between $\left[\mathrm{Cp}(\mathrm{CN})_{5}\right]^{-}$anions that were detected in the crystal structure of compound $\mathbf{5 - C p ( C N})_{5}-\mathbf{1}^{a}$

\begin{tabular}{|cc|}
\hline $\boldsymbol{C g} \cdots \boldsymbol{C g}$ & $\begin{array}{c}\boldsymbol{C g}(\text { centroid }) \cdots \\
\boldsymbol{C g}(\text { centroid })(\AA)\end{array}$ \\
\hline $\boldsymbol{C g} 1 \cdots \boldsymbol{C g} \mathbf{2}^{\mathrm{i}}$ & $4.0336(9)$ \\
$\boldsymbol{C g} 1 \cdots \boldsymbol{C g}^{\mathrm{ii}}$ & $3.7558(9)$ \\
\hline${ }^{a}$ Symmetry codes: (i) $x, y, z ;$ (ii) $-1 / 2+x$, \\
$1 / 2-y, z$. \\
\hline
\end{tabular}

The dihedral angle between the planes through the respective ring atoms of the two $\left[\mathrm{Cp}(\mathrm{CN})_{5}\right]^{-}$ anions in the asymmetric unit is $3.55(5)^{\circ}$. Within each anionic column, consecutive anions are slightly rotated relative to each other (twist angle of $6.37(9)^{\circ}$ ), presumably to relieve some of the energy penalty coming from parallel dipolar cyano groups that typically tend to adopt an antiparallel arrangement. The $\left[\mathrm{Cp}(\mathrm{CN})_{5}\right]^{-}$rings are also tilted by $27.5^{\circ}$ out of the columnar cross- 
sectional planes (which are parallel to the $b \times c$ plane). Due to the tilt, the cross-sectional area $S_{\text {col }}$ of one column of stacked $\left[\mathrm{Cp}(\mathrm{CN})_{5}\right]^{-}$anions along the $a$-axis, surrounded by $\left[\left(\mathrm{C}_{1}\right)_{5} \mathrm{im}\right]^{+}$cations, is reduced from $131.3 \AA^{2}$ (i.e., the columnar area in the planes of the $\left[\mathrm{Cp}(\mathrm{CN})_{5}\right]^{-}$rings, see below) to $(b \times c) / 4=116.468 \AA^{2}$. We note that for the mesophase $\mathrm{M}_{\text {hex }}$ at $240{ }^{\circ} \mathrm{C}$, an $S_{\text {col }}$ value of 131.2 $\AA^{2}$ was derived (see the main text). This implies that the tilt angle of the anions in the mesophase is, on average, close to $0^{\circ}$. Some residual tilt is present within the columns $\left(h_{\text {mol }}>h_{\pi}\right.$, see Table S8 in Section 7), but the hexagonal symmetry that was found by SWAXS implies that it is averaged to zero on the length scale of the columnar domains in the sample, which is in agreement with the homeotropic regions that were observed by polarized-light optical microscopy.

Derivation of the columnar area in the planes of the $\left[\mathrm{Cp}(\mathrm{CN})_{5}\right]^{-}$rings from the single-crystal $X$ -

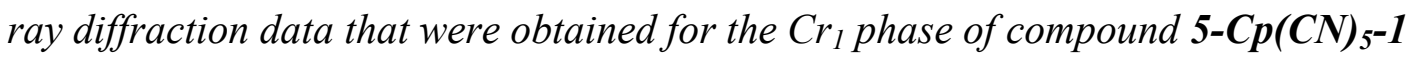

- Periodic stacking of $4\left[\mathrm{Cp}(\mathrm{CN})_{5}\right]^{-}$rings along the $a$-axis, hence: $h_{\text {mol }}=a / 4=3.8320 \AA$

- Tilt angle of $\left[\mathrm{Cp}(\mathrm{CN})_{5}\right]^{-}$rings out of the columnar cross-sectional planes (which are parallel to the $b \times c$ plane): $\psi=27.5^{\circ}$

- Distance between consecutive (or superposed) $\left[\mathrm{Cp}(\mathrm{CN})_{5}\right]^{-}$rings: $h_{\left[\mathrm{Cp}(\mathrm{CN})_{5}\right]^{-}}=h_{\mathrm{mol}} \times \cos (\psi)=$ $(3.8320 \AA) \times \cos \left(27.5^{\circ}\right)=3.40 \AA$

- Cross-sectional area of superposed molecules in the $\left[\mathrm{Cp}(\mathrm{CN})_{5}\right]^{-}$ring plane: $\sigma_{\mathrm{mol}}=$ $V_{\mathrm{mol}} / h_{[\mathrm{Cp}(\mathrm{CN}) 5]^{-}}=\left(V_{\text {cell }} / 16\right) / h_{[\mathrm{Cp}(\mathrm{CN}) 5]^{-}}=\left(446.3 \AA^{3}\right) /(3.40 \AA)=131.3 \AA^{2}$

Note regarding the observed "charge-segregated" arrangement

While "charge-segregated" arrangements were identified in the crystal phases and the mesophase of 5-Cp(CN) $)_{5} \mathbf{1}$ (see also Section 7 below), "charge-by-charge" structures have been reported in the solid states of non-mesomorphic $\left[\mathrm{Cp}(\mathrm{CN})_{5}\right]^{-}$salts with planar, " $\pi$-electronic" tropylium, crystal violet or 4,8,12-tris(n-propyl)-4,8,12-triazatriangulenium $\left(\left[\mathrm{TATA}^{\mathrm{C} 3}\right]^{+}\right)$counterions. $^{20}$ 
7. Small- to wide-angle X-ray scattering (SWAXS) data

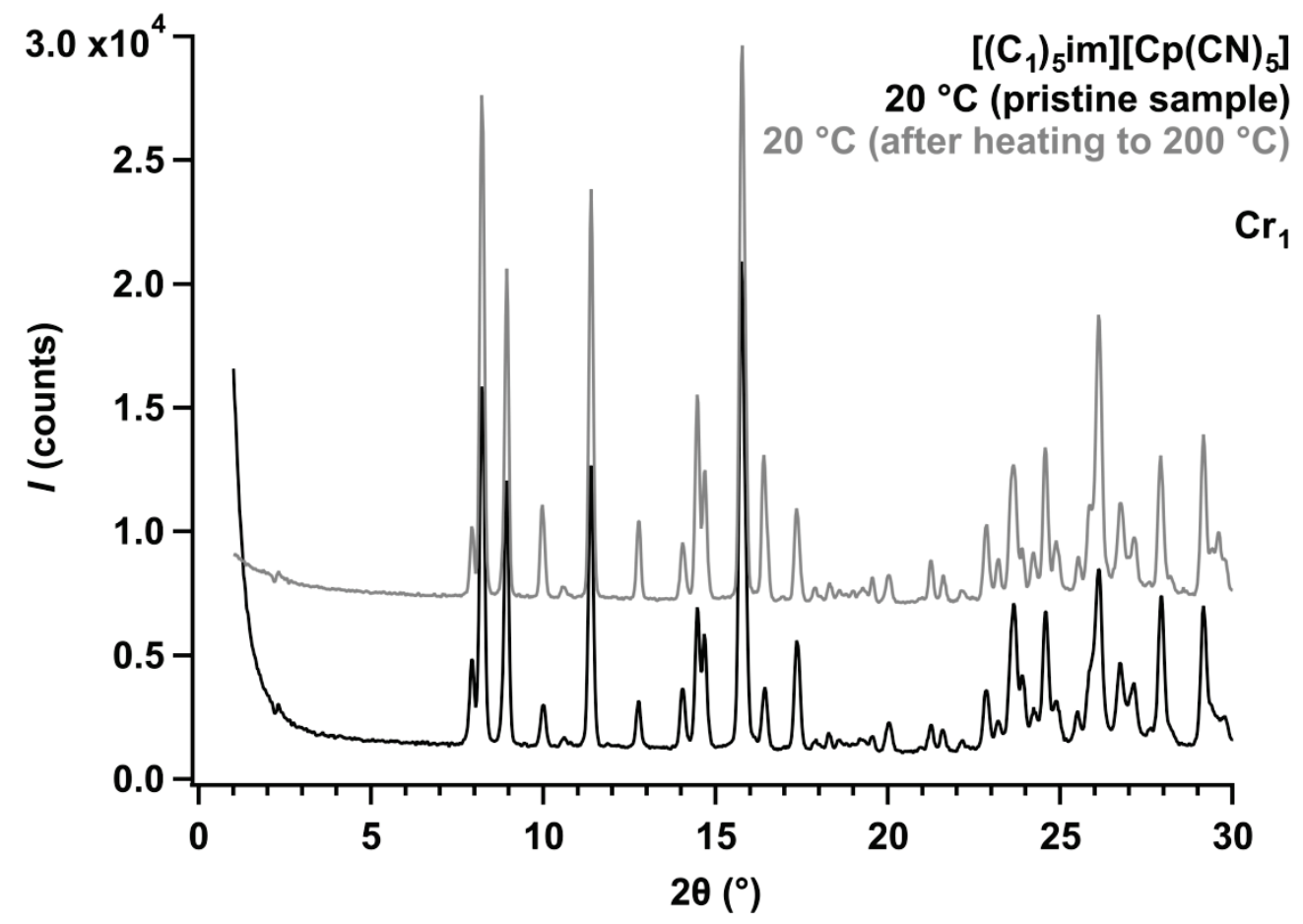

Figure S32. SWAXS patterns that were recorded for the $\mathrm{Cr}_{1}$ phase of compound $\mathbf{5}-\mathbf{C p}(\mathbf{C N})_{5}-\mathbf{1}$ at $20{ }^{\circ} \mathrm{C}$.

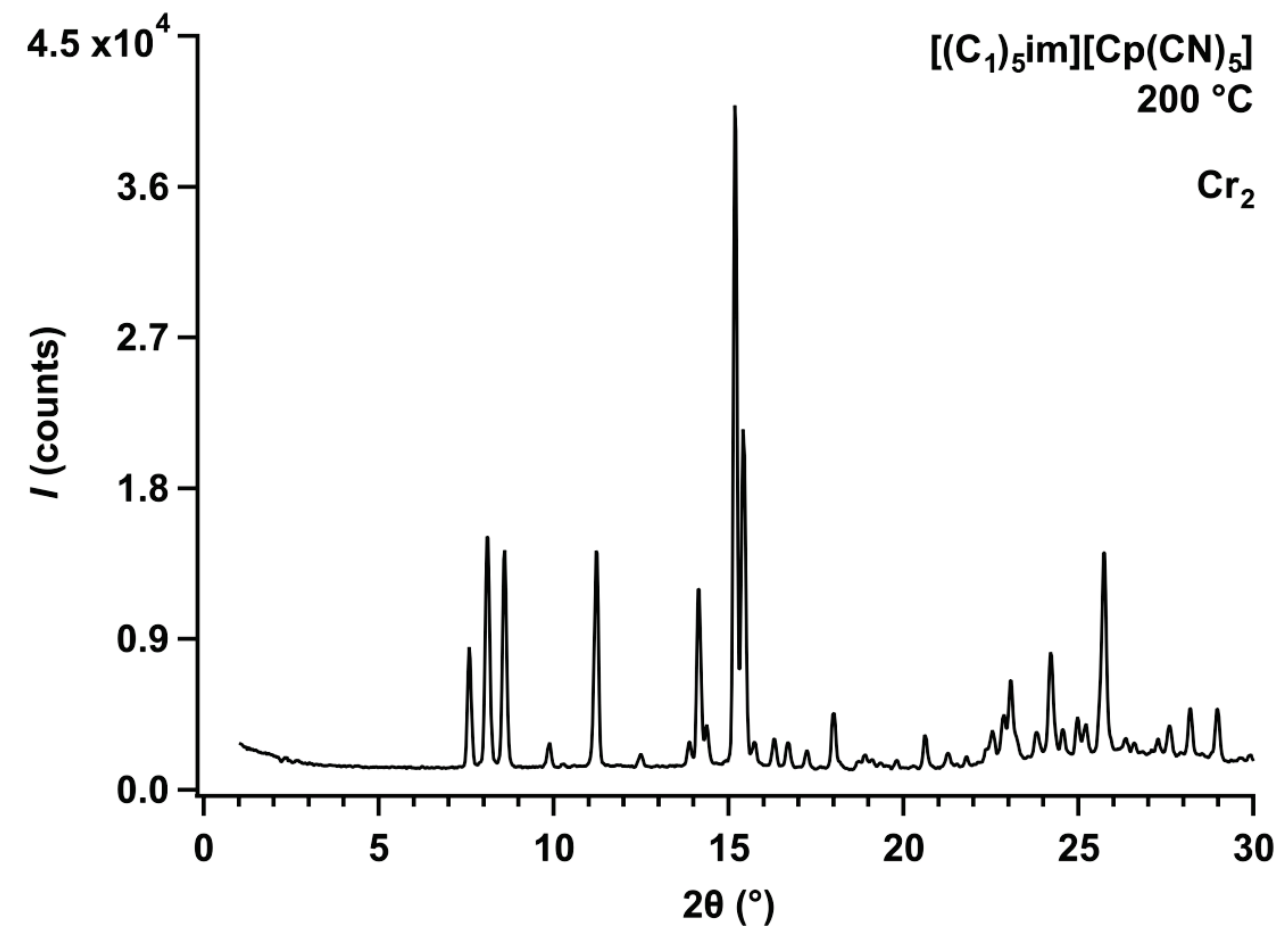

Figure S33. SWAXS pattern that was recorded for the $\mathrm{Cr}_{2}$ phase of compound $\left.\mathbf{5 - C p ( C N )}\right)_{\mathbf{5}} \mathbf{- 1}$ at $200{ }^{\circ} \mathrm{C}$. 
Table S7. Summary of SWAXS data that were recorded for the $\mathrm{Cr}_{2}$ phase adopted by compound 5-Cp(CN) $)_{5} \mathbf{1}$, including calculated structural parameters.

\begin{tabular}{|c|c|c|c|c|c|}
\hline $\begin{array}{l}T=200^{\circ} \mathrm{C}, \mathrm{C} \\
\text { Orthorhombi } \\
a=15.75 \AA, b\end{array}$ & $5 \AA, c=23$. & $=\gamma$ & $75 \AA^{3}$ & $\left.V_{\mathrm{mol}}=498 \AA^{3}\right)$ & \\
\hline $2 \theta_{\text {obs. }}\left({ }^{\circ}\right)^{b}$ & $d_{\text {obs. }}(\AA)^{c}$ & $I^{d}$ & $h k l^{e}$ & $2 \theta_{\text {calcd. }}\left({ }^{\circ}\right)^{b}$ & $d_{\text {calcd. }}(\AA)^{c}$ \\
\hline 7.59 & 11.64 & M & 002 & 7.589 & 11.64 \\
\hline 8.11 & 10.89 & $\mathrm{~S}$ & 020 & 8.123 & 10.875 \\
\hline 8.59 & 10.28 & $\mathrm{~S}$ & 012 & 8.609 & 10.263 \\
\hline 9.87 & 8.950 & W & 120 & 9.876 & 8.949 \\
\hline 11.22 & 7.881 & $\mathrm{~S}$ & 200 & 11.226 & 7.875 \\
\hline 12.48 & 7.086 & W & 122 & 12.466 & 7.095 \\
\hline 13.88 & 6.375 & W & 220 & 13.873 & 6.378 \\
\hline
\end{tabular}




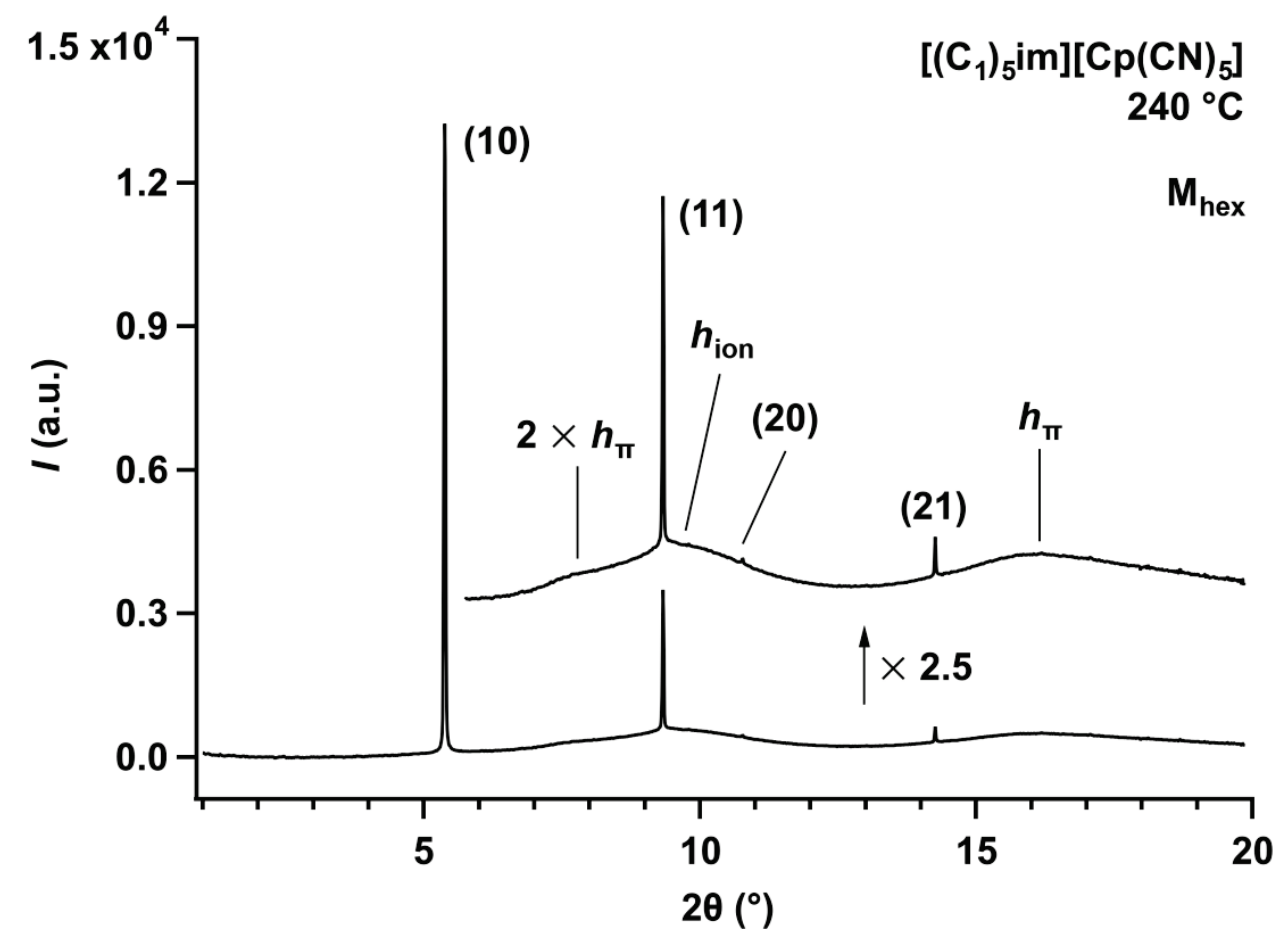

Figure S34. Synchrotron-based SWAXS pattern that was recorded for the $\mathrm{M}_{\text {hex }}$ phase of compound 5$\mathbf{C p}(\mathbf{C N})_{5}-1$ at $240{ }^{\circ} \mathrm{C}$ (the X-ray wavelength used was $1.00 \AA$ ). As the sample showed a pronounced tendency to form oriented domains, the observed intensity ratios were found to vary between different measurements.

Table S8. Summary of SWAXS data that were recorded for the $\mathrm{M}_{\text {hex }}$ phase adopted by compound 5-Cp(CN) $\mathbf{5}-\mathbf{1}$, including calculated structural parameters.

\begin{tabular}{|c|c|c|c|c|c|}
\hline \multicolumn{6}{|c|}{$\begin{array}{l}T=240{ }^{\circ} \mathrm{C}, \mathrm{M}_{\mathrm{hex}}(p 6 \mathrm{~mm}) \\
a_{\mathrm{hex}}=12.31 \AA, V_{\mathrm{mol}} \approx 536( \pm 20) \AA^{3}, S_{\mathrm{col}}=131.2 \AA^{2}, h_{\mathrm{mol}} \approx 4.1 \AA^{a}\end{array}$} \\
\hline $2 \theta_{\text {obs. }}\left({ }^{\circ}\right)^{b}$ & $d_{\text {obs. }}(\AA)^{c}$ & $I^{d}$ & $h k^{e}$ & $d_{\text {calcd. }}(\AA)^{c}$ & $d_{\text {obs. }}-d_{\text {calcd. }}(\AA)$ \\
\hline 5.38 & 10.66 & $\mathrm{VS}(\mathrm{sh})$ & 10 & 10.66 & 0.00 \\
\hline 9.33 & 6.15 & $\mathrm{~S}(\mathrm{sh})$ & 11 & 6.16 & -0.01 \\
\hline 10.77 & 5.33 & $\mathrm{~W}(\mathrm{sh})$ & 20 & 5.33 & 0.00 \\
\hline \multirow[t]{4}{*}{14.26} & 4.03 & $\mathrm{M}(\mathrm{sh})$ & 21 & 4.03 & 0.00 \\
\hline & 7.3 & br & $2 \times h_{\pi}$ & & \\
\hline & 5.9 & br & $h_{\text {ion }}$ & & \\
\hline & 3.6 & br & $h_{\pi}$ & & \\
\hline
\end{tabular}


values refer to the measured and calculated diffraction spacings, respectively. ${ }^{d} I$ is the intensity of each reflection: $\mathrm{VS}=$ very strong, $\mathrm{S}=$ strong, $\mathrm{M}=$ medium, $\mathrm{W}=$ weak, $\mathrm{sh}=$ sharp reflection, $\mathrm{br}$ = broad reflection. As the sample showed a pronounced tendency to form oriented domains, the observed intensity ratios were found to vary between different measurements. ${ }^{e} h k$ are the Miller indices of the reflections. $h_{\pi}$ indicates the center position of the diffuse wide-angle signal that originates from $\pi$-stacking of the $\left[\mathrm{Cp}(\mathrm{CN})_{5}\right]^{-}$anions. The broad scattering signal centered at $\sim 7.3 \AA$ corresponds to $2 \times h_{\pi}$ and is presumably related to the fact that one cation interacts with two stacked anions and turns/shifts more frequently occur for each second anion within the columnar stacks. The higher value of $h_{\mathrm{mol}}$ as compared to $h_{\pi}$ indicates the presence of residual tilt of the anions in the mesophase. The tilt presumably compensates for the space requirements of the surrounding cations. However, as pointed out in Section 6.2, there is no correlation between the tilt-induced orientations of the $\left[\mathrm{Cp}(\mathrm{CN})_{5}\right]^{-}$stacks that are arranged on a 2D hexagonal lattice, and the average tilt angle is close to zero. $h_{\text {ion }}$ indicates the center position of the broad scattering signal that is related to the average distances between the $\left[\left(\mathrm{C}_{1}\right)_{5} \text { im }\right]^{+}$cations in the cationic continuum. 


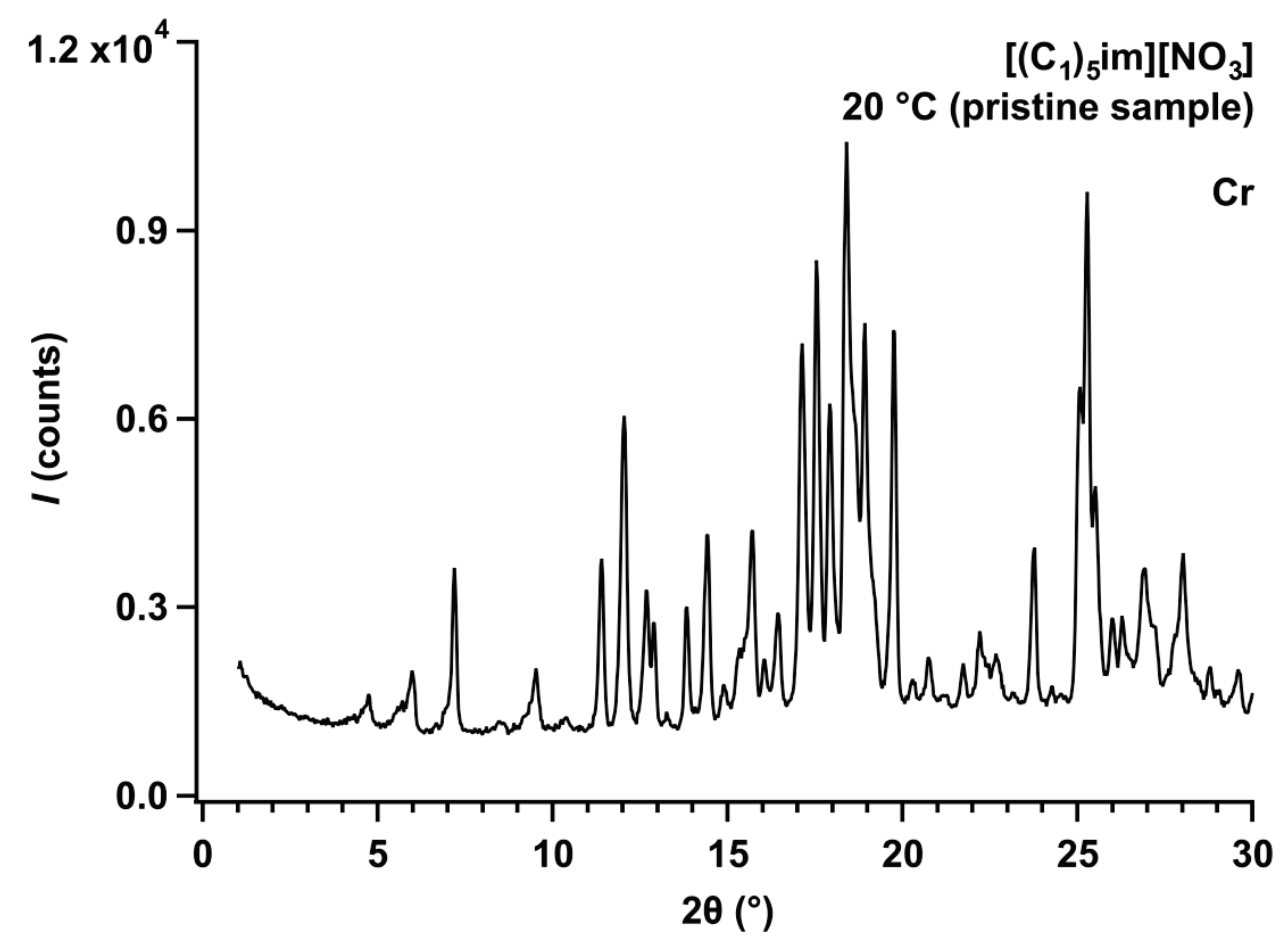

Figure S35. SWAXS pattern that was recorded for the Cr phase of compound 5-NO $\mathbf{O}_{3}-1$ at $20{ }^{\circ} \mathrm{C}$.

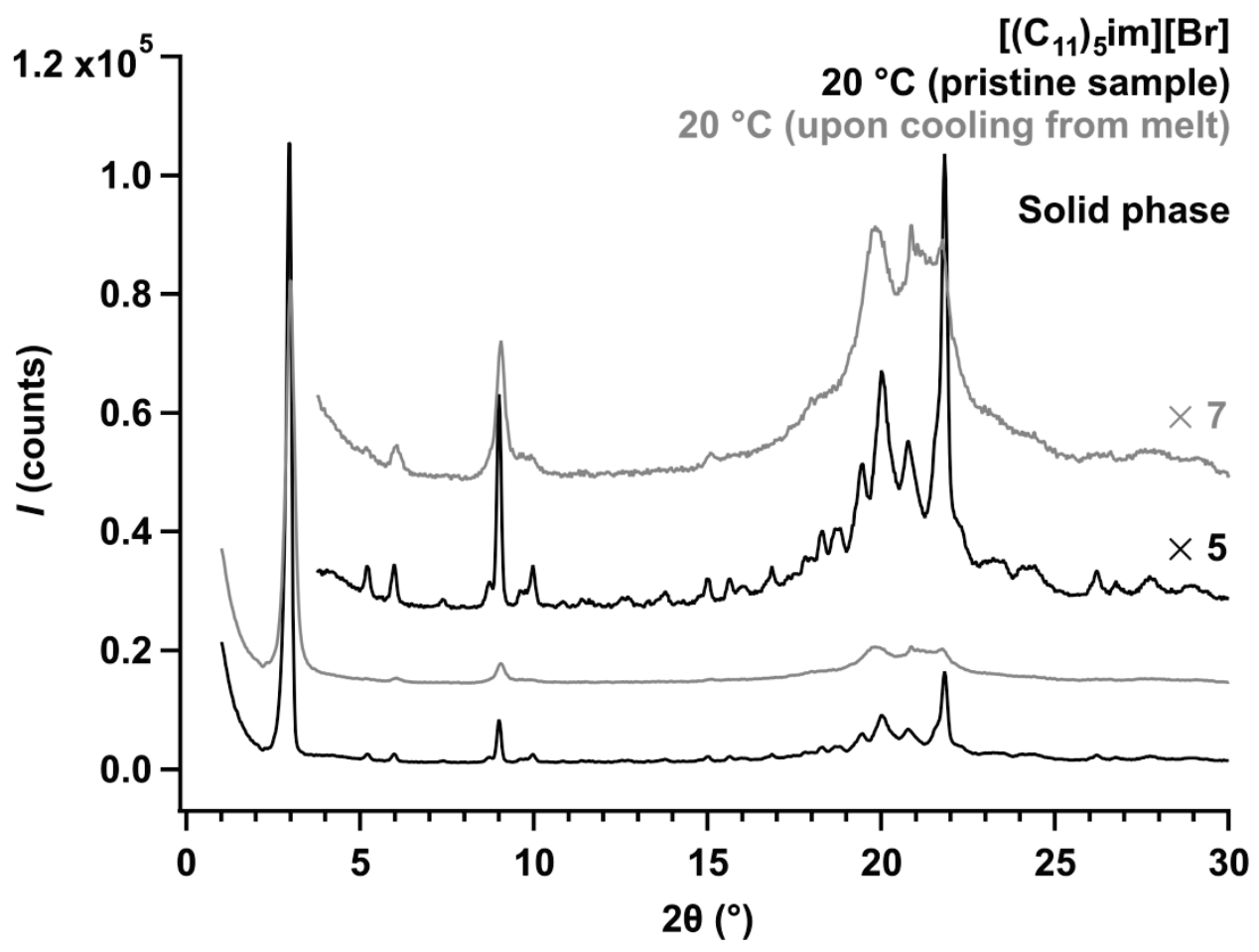

Figure S36. SWAXS patterns that were recorded for the solid phase of compound 5-Br-11 at $20^{\circ} \mathrm{C}$. 


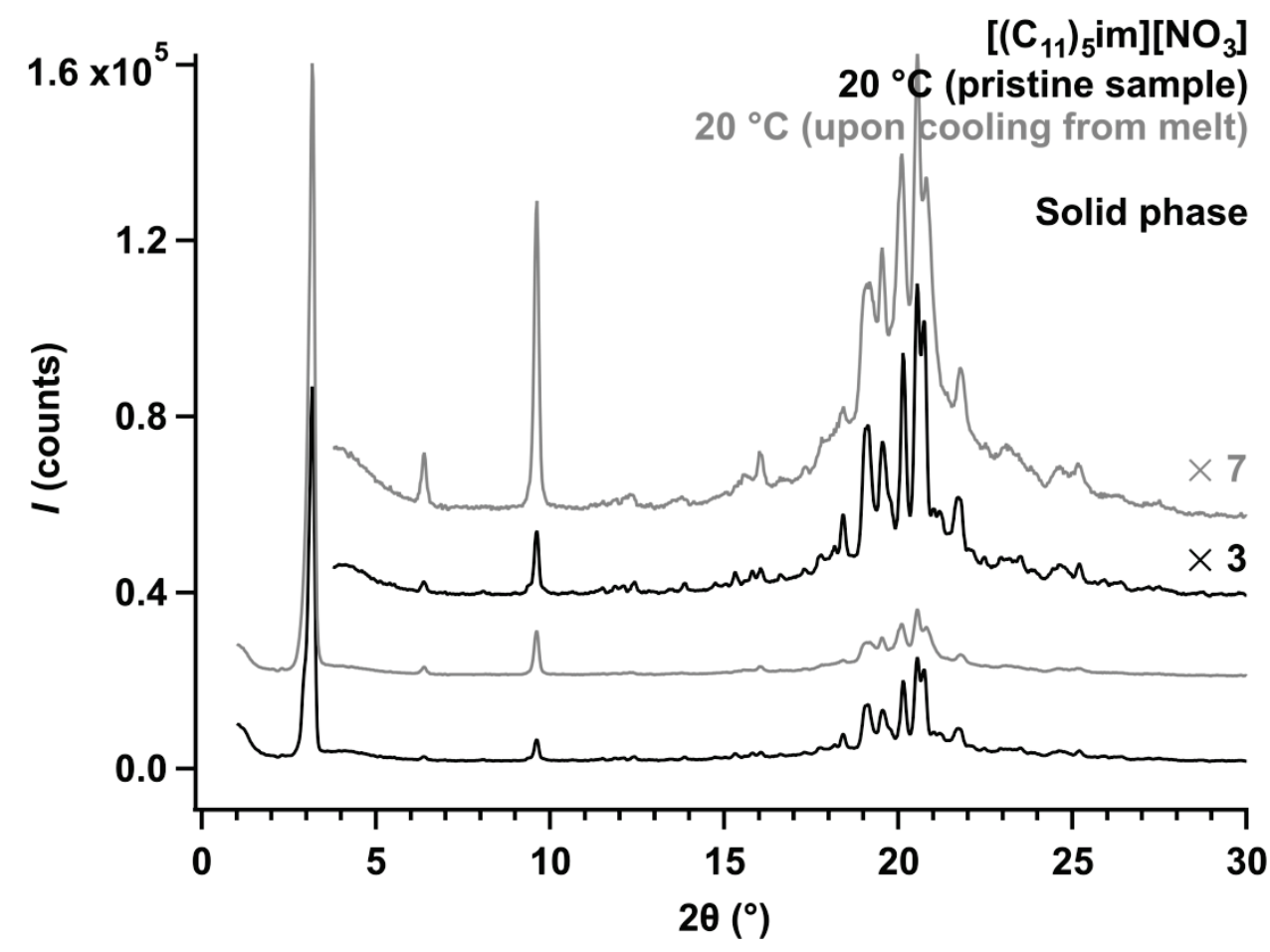

Figure S37. SWAXS patterns that were recorded for the solid phase of compound 5-NO $\mathbf{O}_{3}-11$ at $20{ }^{\circ} \mathrm{C}$.

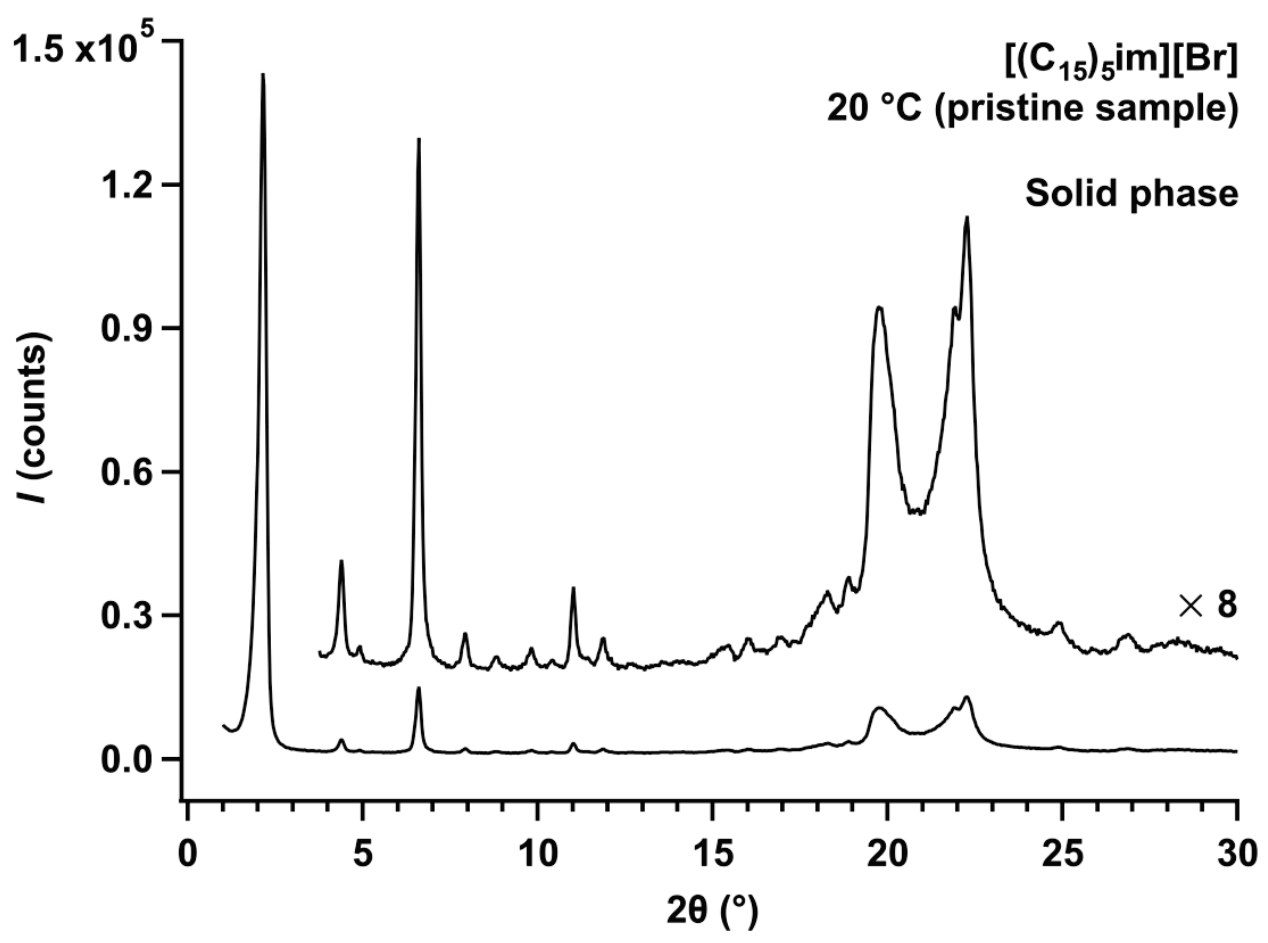

Figure S38. SWAXS pattern that was recorded for the solid phase of compound 5-Br-15 at $20^{\circ} \mathrm{C}$. 


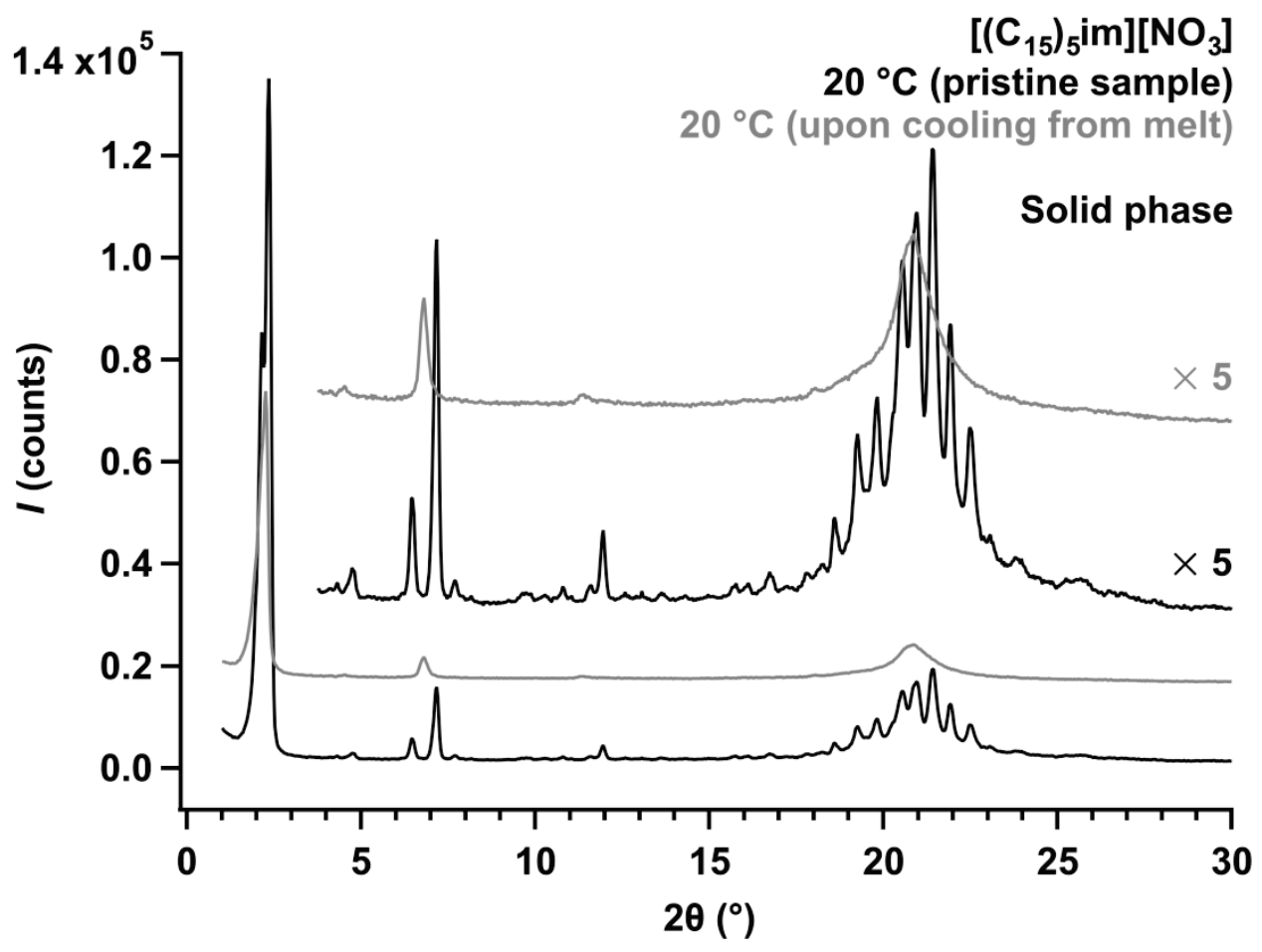

Figure S39. SWAXS patterns that were recorded for the solid phase of compound 5- $\mathrm{NO}_{3}-15$ at $20^{\circ} \mathrm{C}$. 


\section{Supplementary note: Comparison with the thermal characteristics of long-chain- substituted $\left[\mathrm{C}_{n} \mathrm{mim}\right][\mathrm{X}]$ and $\left[\left(\mathrm{C}_{n}\right)_{2} \mathrm{im}\right][\mathrm{X}]$ salts}

1-Alkyl-3-methylimidazolium $\left(\left[\mathrm{C}_{n} \mathrm{mim}\right]^{+}\right)$and 1,3-dialkylimidazolium $\left(\left[\left(\mathrm{C}_{n}\right)_{2} \mathrm{im}\right]^{+}\right)$ salts are known ${ }^{21}$ to self-organize into thermotropic smectic A (SmA) mesophases when outfitted with sufficiently long alkyl substituents in order to achieve a certain degree of amphiphilicity (e.g., $\left[\mathrm{C}_{n} \mathrm{mim}\right]\left[\mathrm{BF}_{4}\right]: n \geq 12,{ }^{22}\left[\left(\mathrm{C}_{n}\right)_{2} \mathrm{im}\right][\mathrm{Cl}] \cdot \mathrm{H}_{2} \mathrm{O}: n \geq 8^{23}$ ). The presence of only two alkyl substituents promotes the lateral association of ionic moieties and molten chains into nanosegregated layers, whose alternation generates the smectic periodicity. ${ }^{24}$ The disappearance of mesomorphic properties for the compounds with five radiating alkyl substituents $\left(\mathbf{5 - X}-\boldsymbol{n}\left(\mathrm{X}^{-}=\mathrm{Br}^{-},\left[\mathrm{NO}_{3}\right]^{-},\left[\mathrm{Cp}(\mathrm{CN})_{5}\right]^{-} ; n=7,11,15\right)\right)$ is indicative of a hampered lateral association of ions due to the intercalation of chain segments and the modified cross-sectional areas of the molecules. Besides geometrical constraints, the larger overall volume occupied by the five alkyl substituents and the resulting dilution of ionic domains must be considered.

To deconvolute such contributions, compare salts with similar numbers of alkyl chain carbon atoms, such as $\left[\left(\mathrm{C}_{18}\right)_{2} \mathrm{im}\right][\mathrm{Br}]$, which adopts a $\mathrm{SmA}$ phase ranging over $100{ }^{\circ} \mathrm{C},{ }^{23}$ to 5-Br-7, which does not exhibit an anisotropic LC mesophase even though it has a lower melting point. Such comparisons uncover a detrimental effect of symmetric pentasubstitution on the mesomorphic properties of imidazolium salts. While one could envision a lamellar LC mesophase that features alternating ionic monolayers and aliphatic sublayers and an equal, statistical distribution of the molten chains between adjacent aliphatic sublayers, ${ }^{25}$ compact packing of the ionic moieties would be prevented in such an arrangement by intercalation of chain segments close to the imidazolium rings. The frustration is aggravated by the overall space requirements of the alkyl chains, which impose a significant lateral expansion to the whole lamellar sequence. The geometric constraints could have resulted in the formation of ionic wire-like structures that loosely associated into sheets. Alternatively, the sheets could have dissociated into individual wires that occupied the nodes of a two-dimensional (2D) columnar-like lattice. If such structures had existed, the cohesion between the ionic segments would have been low and relatively low clearing temperatures may be expected. However, the complete absence of 
mesomorphic properties, even for the amphiphilic salts with planar $\left[\mathrm{NO}_{3}\right]^{-}$or $\left[\mathrm{Cp}(\mathrm{CN})_{5}\right]^{-}$ counterions, was not anticipated. 


\section{References and notes}

(1) Fulmer, G. R.; Miller, A. J. M.; Sherden, N. H.; Gottlieb, H. E.; Nudelman, A.; Stoltz, B. M.; Bercaw, J. E.; Goldberg, K. I. NMR Chemical Shifts of Trace Impurities: Common Laboratory Solvents, Organics, and Gases in Deuterated Solvents Relevant to the Organometallic Chemist. Organometallics 2010, 29, 2176-2179.

(2) Preston-Thomas, H. The International Temperature Scale of 1990 (ITS-90). Metrologia 1990, 27, 3-10.

(3) Archer, D. G.; Rudtsch, S. Enthalpy of Fusion of Indium: A Certified Reference Material for Differential Scanning Calorimetry. J. Chem. Eng. Data 2003, 48, 1157-1163.

(4) Legendre, B.; Sghaier, M. Curie Temperature of Nickel. J. Therm. Anal. Calorim. 2011, 105, 141-143.

(5) Wang, D.; Richter, C.; Rühling, A.; Drücker, P.; Siegmund, D.; Metzler-Nolte, N.; Glorius, F.; Galla, H.-J. A Remarkably Simple Class of Imidazolium-Based Lipids and Their Biological Properties. Chem. Eur. J. 2015, $21,15123-15126$.

(6) Sakai, T.; Seo, S.; Matsuoka, J.; Mori, Y. Synthesis of Functionalized Tetracyanocyclopentadienides From Tetracyanothiophene and Sulfones. J. Org. Chem. 2013, 78, 10978-10985.

(7) Roth, C.; Peppel, T.; Fumino, K.; Köckerling, M.; Ludwig, R. The Importance of Hydrogen Bonds for the Structure of Ionic Liquids: Single-Crystal X-Ray Diffraction and Transmission and Attenuated Total Reflection Spectroscopy in the Terahertz Region. Angew. Chem., Int. Ed. 2010, 49, 10221-10224.

(8) Kohnen, G.; Tosoni, M.; Tussetschlager, S.; Baro, A.; Laschat, S. Counterion Effects on the Mesomorphic Properties of Chiral Imidazolium and Pyridinium Ionic Liquids. Eur. J. Org. Chem. 2009, 5601-5609.

(9) Barberá, J.; Rakitin, O. A.; Ros, M. B.; Torroba, T. Breaking the Mold of Discotic Liquid Crystals. Angew. Chem., Int. Ed. 1998, 37, 296-299.

(10) Basurto, S.; García, S.; Neo, A. G.; Torroba, T.; Marcos, C. F.; Miguel, D.; Barberá, J.; Ros, M. B.; de la Fuente, M. R. Indene and Pseudoazulene Discotic Liquid Crystals: A Synthetic and Structural Study. Chem. Eur. J. 2005, 11, 5362-5376.

(11) See also: Kuhn, N.; Henkel, G.; Kreutzberg, J. Synthese und Reaktionen von 1,2,4,5-Tetramethylimidazol; die Kristallstruktur von Pentamethylimidazolium-iodid. Z. Naturforsch. B 1991, 46b, 1706-1712.

(12) CrystalClear-SM Expert (version 2.1 b45), Rigaku Americas Corp.: The Woodlands, TX, USA, 2015.

(13) ABSCOR, T. Higashi, Rigaku Corp.: Tokyo, Japan, 1995.

(14) Dolomanov, O. V.; Bourhis, L. J.; Gildea, R. J.; Howard, J. A. K.; Puschmann, H. OLEX2: a Complete Structure Solution, Refinement and Analysis Program. J. Appl. Crystallogr. 2009, 42, 339-341.

(15) Sheldrick, G. M. A Short History of SHELX. Acta Cryst. A 2008, 64, 112-122.

(16) Sheldrick, G. M. Crystal Structure Refinement With SHELXL. Acta Cryst. C 2015, 71, 3-8.

(17) Spek, A. L. Structure Validation in Chemical Crystallography. Acta Cryst. D 2009, 65, 148-155.

(18) In Platon we used the default parameter values for defining hydrogen bonds: $d(\mathrm{D} \cdots \mathrm{A})<\left[R_{\mathrm{vdW}}(\mathrm{D})+R_{\mathrm{vdW}}(\mathrm{A})+\right.$ $0.50 \AA] ; d(\mathrm{H} \cdots \mathrm{A})<\left[R_{\mathrm{vdW}}(\mathrm{H})+R_{\mathrm{vdW}}(\mathrm{A})-0.12 \AA\right] ; \mathrm{D}-\mathrm{H} \cdots \mathrm{A}>100.0^{\circ}(\mathrm{D}=$ hydrogen-bond donor atom; $\mathrm{A}=$ hydrogen-bond acceptor atom; $R_{\mathrm{vdW}}=$ van der Waals radius). 
(19) Bourhis, L. J.; Dolomanov, O. V.; Gildea, R. J.; Howard, J. A.; Puschmann, H. The Anatomy of a Comprehensive Constrained, Restrained Refinement Program for the Modern Computing Environment - Olex2 Dissected. Acta Cryst. A 2015, 71, 59-75.

(20) Bando, Y.; Haketa, Y.; Sakurai, T.; Matsuda, W.; Seki, S.; Takaya, H.; Maeda, H. Ion-Pairing Assemblies Based on Pentacyano-Substituted Cyclopentadienide As a Pi-Electronic Anion. Chem. Eur. J. 2016, 22, 78437850 .

(21) (a) Goossens, K.; Lava, K.; Bielawski, C. W.; Binnemans, K. Ionic Liquid Crystals: Versatile Materials. Chem. Rev. 2016, 116, 4643-4807; (b) Mansueto, M.; Laschat, S. Ionic Liquid Crystals. In Handbook of Liquid Crystals. Volume 6: Nanostructured and Amphiphilic Liquid Crystals; 2nd ed.; Goodby, J. W., Collings, P. J., Kato, T., Tschierske, C., Gleeson, H., Raynes, P., Eds.; Wiley-VCH: Weinheim, 2014; pp 231-280; (c) Fernandez, A. A.; Kouwer, P. H. J. Key Developments in Ionic Liquid Crystals. Int. J. Mol. Sci. 2016, 17, 731; (d) Kato, T.; Yoshio, M.; Ichikawa, T.; Soberats, B.; Ohno, H.; Funahashi, M. Transport of Ions and Electrons in Nanostructured Liquid Crystals. Nat. Rev. Mater. 2017, 2, 17001.

(22) Holbrey, J. D.; Seddon, K. R. The Phase Behaviour of 1-Alkyl-3-Methylimidazolium Tetrafluoroborates; Ionic Liquids and Ionic Liquid Crystals. J. Chem. Soc., Dalton Trans. 1999, 2133-2139.

(23) Rondla, R.; Lee, C. K.; Lu, J. T.; Lin, I. J. B. Symmetrical 1,3-Dialkylimidazolium Based Ionic Liquid Crystals. J. Chin. Chem. Soc. 2013, 60, 745-754.

(24) Bradley, A. E.; Hardacre, C.; Holbrey, J. D.; Johnston, S.; McMath, S. E. J.; Nieuwenhuyzen, M. Small-Angle X-Ray Scattering Studies of Liquid Crystalline 1-Alkyl-3-Methylimidazolium Salts. Chem. Mater. 2002, 14, $629-635$.

(25) Symmetrical tetrakis(n-alkyl)ammonium halides (which are not LC) were previously found to crystallize as consecutive ionic monolayers separated by aliphatic sublayers of non-interdigitating alkyl chains, with the four equivalent chains of each molecule adopting either a "biradial" (i.e., chains paired two-by-two intramolecularly) or a "tetraradial" (i.e., chains unassociated intramolecularly) shape depending on the chain lengths and the anion size [see: Abdallah, D. J.; Bachman, R. E.; Perlstein, J.; Weiss, R. G. Crystal Structures of Symmetrical Tetra- $n$-Alkyl Ammonium and Phosphonium Halides. Dissection of Competing Interactions Leading to "Biradial" and "Tetraradial" Shapes. J. Phys. Chem. B 1999, 103, 9269-9278; Abdallah, D. J.; Lu, L.; Cocker, T. M.; Bachman, R. E.; Weiss, R. G. The Crystalline and Liquid Crystalline Structures of BenzylTri-Octadecylammonium Bromide Complete the Puzzle: How Do Group VA Halide Salts with One-Four Long n-Alkyl Chains Pack? Liq. Cryst. 2000, 27, 831-837]. 